\title{
MULTIRESIDUE ANALYSIS OF OVER 233 PESTICIDES IN CUCUMBER AND GRAPEFRUIT SAMPLES USING A QUEChERS AND GAS CHROMATOGRAPHY-TANDEM MASS SPECTROMETRY-BASED METHOD
}

\author{
HEPSAĞ, F. \\ Korkut Ata University, School of Applied Sciences, Department of Food Technology \\ Kadirli Campus, 80750 Osmaniye, Turkey \\ ORCID ID: 0000-0002-3688-4106; e-mail: fatmahepsag@osmaniye.edu.tr \\ phone: +90-328-888-0090; fax: +90-328-888-0091 \\ (Received $15^{\text {th }}$ Feb 2019; accepted $8^{\text {th }}$ Apr 2019)
}

\begin{abstract}
This report describes the 233 pesticide residue in cucumber and grapefruit sold in Turkey. In total, 680 fruit and vegetable samples were analyzed by liquid chromatography connected to tandem mass spectrometry (LC-MS / MS) with the device in which gas chromatography to mass spectrometry (GCMS) units work together. In cucember, limit of detection (LOD) varied from $0.17 \mu \mathrm{g} \mathrm{kg}^{-1}$ to $11.8 \mu \mathrm{g} \mathrm{kg}^{-1}$ and the limit of quantifications (LOQ) varied from $0.42 \mu \mathrm{g} \mathrm{kg}^{-1}$ to $39.35 \mathrm{ug} \mathrm{kg}^{-1}$; whereas for grapefruit, $\mathrm{LOD}$, and LOQ values varied from $0.13 \mu \mathrm{g} \mathrm{kg}^{-1}$ to $11.80 \mu \mathrm{g} \mathrm{kg}^{-1}, 0.42 \mu \mathrm{g} \mathrm{kg}^{-1}$, and $39.35 \mu \mathrm{g} \mathrm{kg}^{-1}$, respectively. Recoveries obtained for each pesticide in both matrices ranged between $77.87 \%$ and $104.15 \%$. Relative standard deviation (RSD) values calculated were $>20 \%(24.32 \%)$ for clofentazine in cucember at $20.0 \mathrm{mg} \mathrm{kg}^{-1}$ and $(21.36 \%)$ for propyzamide, (29.11\%) for fludioxonil grapefruit at $100 \mu \mathrm{g}$ $\mathrm{kg}^{-1}$. For the other compounds, at both concentration levels evaluated in cucumber and grapefruit, the RSD values were $<20 \%$. The extended measurement uncertainty for individual pesticides ranged from 0.208 to 0.434 . In general, $11.6 \%$ of cucumbers and $13.8 \%$ of grapefruits contained at least one detectable residue, but levels were below the EU legal limits.
\end{abstract}

Keywords: QuEChERS, GS-MS, LC-MS / MS, pesticide, grapefruit, cucumber

\section{Introduction}

Pesticide is used in all kinds of chemical substances and preparations used in agricultural researches and applications. The pesticide may be a chemical agent, a biological agent such as a virus or a bacterium, an antimicrobial, a disinfectant or any other vehicle. Although the use of pesticides may have some benefits, it can cause problems for humans and other living things due to their potential toxicity. Such as pesticides, some of which include biopreparations, insect and plant growth regulators, pheromones (hormone-bearing) and other attractants, feed-blockers, repellents (insect repellents), traps, plant activators, preparations used in the treatment of physiological diseases (Epstein and Zhang, 2014).

Turkey is the 4th largest fresh fruit and vegetable producers in the world. As of 2015 , about $37 \%$ of the total production of the citrus group consisting of lemon, orange, mandarin and grapefruit (3.7 million tons) and cucumber (100 thousand tons) accounted for approximately $2.8 \%$ of exports. Almost all of the total value of exported fresh fruits and vegetables has entered the EU, Russian Federation, Ukraine and Iraqi markets (TUIK, 2015; FAO, 2015).

In food, the permissible residual limit values are as low as in parts per million $(\mathrm{ppm})$ and in parts per billion (ppb). Therefore, it is accepted that the most effective approach in pesticide residue analysis is multiresidue analyzes. In recent years, many different methods have been successfully used in the detection of pesticide residues in 
foods, especially fruits and vegetables. In order to use these methods gas chromatography (GC), liquid chromatography (LC), coupled to mass selective detectors (MS), many new sample preparation techniques and extraction methods have been developed. Many sample preparation techniques for the detection of many pesticides used in vegetables and fruits, including solid-phase extraction (SPE) (Campone et al., 2018; Chan et al., 2018), solid-phase microextraction (SPME) (Li et al., 2019; Dong et al., 2019), accelerated solvent extraction (ASE) (Su et al., 2018; Stachniuk, 2018), supercritical fluid extraction (SFE) (Alvarez et al., 2019; Khan et al., 2018), matrix solid-phase dispersion (MSPD) (Liu et al., 2018; Jiang et al., 2018), microwave-assisted extraction (MAE) (Du et al., 2018; Wang et al., 2018), membrane extraction (Salemi et al., 2019), gel permeation chromatography (GPC) (Zhu et al., 2019), and QuEChERS (quick, easy, cheap, effective, rugged and safe) method (Tankiewicz, 2019), have been used. Within these techniques, QuEChERS has become more preferred in recent years, because it reduces the use of solvents in accordance with the 'green chemistry' principles, and provides a simple, inexpensive, fast and reliable result.

In this study, 205 pesticides of different classes, which are suitable for LC-MS/MS analysis, and 28 pesticides for GC-MS analysis, were discussed. In our country, pesticide use is still indispensable within the framework of integrated pest management principles. Selected pesticides, widely used in the production stages of fruits and vegetables in Turkey. Some of the selected pesticides may cause acute and chronic diseases due to their toxicity (Kumar et al., 2018).

In Turkey, along with concepts such as food security, have been a lot of studies in a very food production due to the increased use of pesticides. For example, tomatoes (Cengiz et al., 2018), baby foods (Kilic et al., 2018), oranges (Golge and Kabak, 2015), fish products (Polat et al., 2018), apricots (Ersoy et al., 2018), olive oil (Razzaghi et al., 2018) and milk (Yıldırım et al., 2018). However, there are very little data about the presence and levels of pesticide residues in cucumber and grapefruit consumed in Turkey.

This report aims to determine multi-residue pesticides in cucumber and grapefruits consumed in Turkey, monitor the compliance of the fruit and vegetables with the legal limits for target pesticide residues. In this context, the validated analytical method based on gas chromatography to mass spectrometry (GS-MS) and liquid hromatography coupled with tandem mass spectrometry (LC-MS/MS) was applied to 680 samples for a total of 233 pesticides for the periods 2015-2017.

\section{Material and Metod}

\section{Sample collection}

A total of 680 fruit and vegetable samples, including 330 cucumber and grapefruits 350 samples, were obtained from three provinces of Turkey, namely Hatay, İskenderun, Adana, Mersin and Antalya. Approximately $2 \mathrm{~kg}$ of cucumber and grapefruit samples were suplied from bazaar, supermarkets (2015-2017). The vegetables were transported quickly to the laboratory, chopped and homogenised with a home food processor (Robot Coupe model R10, Turkey). The chopped samples were then stored at $-18^{\circ} \mathrm{C}$ until sample preparation step. 


\section{Reagents and chemicals}

Standards of pesticides detected in GC-MS were supplied from ChemService (West Chester, PA, USA) and Standards of pesticides detected in LC-MS/MS from Sigma Aldrich (St. Louis, MO, USA). All standards were of $\geq 98 \%$ purity. Stock solution of multistandard mixture at a concentration of $10 \mathrm{mg} \mathrm{L}^{-1}$ for each substance was dissolved in acetonitrile and kept at $-18^{\circ} \mathrm{C}$ in the dark. This solution was used for preparation of matrix-matched calibration standards at concentrations varying from 0.1 to $100 \mu \mathrm{g} \mathrm{kg}^{-1}$ and for spiking the QuEChERS extracts in validation study.

Acetone, acetonitrile and methanol (GS-MS and LC-MS/MS grade), Isooctane, cyclohexane, ethyl acetate, magnesium sulphate, sodium acetat, glacial acetic acid, formic acid and ammonium formate (purity $\geq 99.9 \%$ ) were supplied from Sigma Aldrich (Stenheim, Germany). Helium (purity $\geq 99.9 \%$ ) was supplied by Praxair (Colima, Mexico). Ultrapure water of $18.2 \mathrm{M} \Omega$ was purchased using a Milli Q water purification system (Milford, MA, USA). The primary-secondary amine (PSA) was supplied from Supelco (Darmstadt, Germany).

\section{Sample preparation}

Pesticide residues were extracted from cucumber and grapefruit samples using QuEChERS protocol according to AOAC official method (AOAC, 2007) with some changes. Cucumber representing high water content product groups, grapefruit with high acid and high water content groups were selected as samples for this study. These vegetable and fruit were selected because of their economic importance in export. Fifteen grams of homogenised sample was placed in to a $50 \mathrm{~mL}$ polypropylene tube, then $15 \mathrm{~mL}$ methanol-acetic acid $(99: 1, \mathrm{v} / \mathrm{v}), 6 \mathrm{~g} \mathrm{MgSO}_{4}$ and $1.5 \mathrm{~g}$ sodium acetate were added. The mixture was shaken vigorously by vortex mixer (Vortex Jr. Mixer from American Scientific Products, catalog no. S82251-1) for $1 \mathrm{~min}$ and centrifuged (Eppendorf 022628225 5804R Centrifuge with A-2-DWP-Plate, Germany) for 2 min at $2000 \mathrm{rpm}$. Later, $8 \mathrm{~mL}$ of the upper methanol phase was added in a $15 \mathrm{~mL}$ polypropylene tube containing $900 \mathrm{mg}$ of MgSO4 and $150 \mathrm{mg}$ of PSA. The mixture was vortexed for $1 \mathrm{~min}$, followed by centrifugation for $2 \mathrm{~min}$ at $2000 \mathrm{rpm}$. For each sample, $200 \mu \mathrm{L}$ was injected into GS-MS and LC-MS / MS.

For GC-MS, $0.010,0.025,0.05,0.1,0.2,0.5$ and $1 \mathrm{ppm}$ prepared standards were given to the device and the corresponding area and retention time were determined. Calibration curve is obtained by plotting the area graph against concentration. For LC-MS/MS, 0.005, 0.010, 0.020, 0.040, 0.080 and $0.160 \mathrm{ppm}$ prepared standards were delivered to the device and the area corresponding to these concentrations and retention time were determined. The fortificated samples were released to equilibrate for 80 minutes before treatment. Blank samples were also prepared according this procedure.

\section{GC-MS conditions}

Analyses were carried out with a Agilent GC-MS, 5975C inert MSD Series gas chromatograph equipped with an MS detector. The system was equipped with capillary column (Rtx-440 GC). Oven start temperature was set at $110^{\circ} \mathrm{C}$, increased to $250^{\circ} \mathrm{C}$ at a rate of $35^{\circ} \mathrm{C} / \mathrm{min}$, increased again at a rate of $40^{\circ} \mathrm{C} / \mathrm{min}$ to $280^{\circ} \mathrm{C}$, this temperature was kept for 3 minutes. The total run time was 5.5 minutes. One $\mathrm{mL}$ of sample was injected in split mode (2:1). The injector temperature was set at $250^{\circ} \mathrm{C}$. 


$$
-6890-
$$

The helium carrier gas flow was $1.1 \mathrm{~mL} / \mathrm{min}$. The mass spectrometer was operated in electron impact ionization mode at $70 \mathrm{eV}$ of collision energy with 2 minute solvent delay to prevent damage to the filament of the ion source. The temperature of the ion source, manifold, and transfer line was set at $250^{\circ} \mathrm{C}, 40^{\circ} \mathrm{C}$, and $280^{\circ} \mathrm{C}$, respectively. For qualitative analysis a full scan from 75 mass/charge $(\mathrm{m} / \mathrm{z})$ to 280 mass/charge $(\mathrm{m} / \mathrm{z})$ was applied. For quantitative pesticide analysis preferred ion monitoring (SIM) mode was used.

\section{LC-MSMS conditions}

Analyses were carried out with a ThermoQuest Surveyor MS Pump and autosampler. Chromatographic separation was carried out on a Waters C18 analytical column Phenomenex LUNA $5 \mu \mathrm{m}$ C18 150 x $2 \mathrm{~mm}$ from Phenomenex (Torrance, CA, USA). The system was controlled by Xcalibur Home Page, Version 1.2 software. The column temperature was set at $40^{\circ} \mathrm{C}$. The solvent systems used were (A) $0.1 \%$ acetic acid and $10 \mathrm{mM}$ ammonium acetate in waterand (B) $0.1 \%$ acetic acid and $10 \mathrm{mM}$ ammonium acetate in 95:5 CH3CN:Water. A flow rate of $200 \mathrm{uL} \mathrm{min}{ }^{-1}$ and an injection volume of $10 \mu \mathrm{L}$ were applied.

The MS was operated using a Negative Ion Electrospray - with metal needle option. The metal needle was connected with a zero dead volume union to the fused silica capillary delivering the mobile phase to the ESI source. Device parameters optimized for MS are as follows. Precursor ion (m/z): 321, product ions (m/z): 257, 194, 176, 152, spray voltage: $1.5 \mathrm{kV}$, collision voltage: $26 \mathrm{~V}$, source offset voltage: 5 $\mathrm{V}$, electron multiplier voltage: $1.27 \mathrm{kV}$, capillary temperature: $350 \mathrm{C}, \mathrm{N}_{2}$ sheath gas: 80 arbitrary units, N2 auxiliary gas: 35 psi, collision gas: Ar.

\section{Method validation}

The proposed method was validated following the European SANTE/11945/2015 Guideline (European Commission, 2015). The established GC-MS and LCMS-MS method were validated in terms of its linearity, limit of detection (LOD), limit of quantification (LOQ), selectivity and recovery. Linearity was evaluated by constructing calibration curves with standard solutions.

\section{Limit of detection (LOD), limit of quantification (LOQ)}

For detection limit of detection (LOD) and limit of quantification (LOQ) operation; In GC-MS, $20 \mu \mathrm{g} \mathrm{kg}^{-1} \mathrm{~kg}$ and LC-MS/MS $10 \mu \mathrm{g} \mathrm{kg}^{-1}$ recovery was performed. 205 pesticides were injected 10 times with the LC-MS/MS device and 28 pesticide GC-MS devices. The LOD values and LOQ values were calculated according to the following formula: $\mathrm{LOD}=3 \mathrm{~s}, \mathrm{LOQ}=10 \mathrm{~s}$, s: Standard deviation.

\section{Linear measurement range}

In order to determine the linear measurement range; Standards for 10 different concentrations $\left(5,10,20,40,80,160 \mu \mathrm{g} \mathrm{kg} \mathrm{kg}^{-1}\right)$ for LC-MS / MS, 7 different concentrations for GC-MS $\left(10,25,50,100,200,500,1000 \mu \mathrm{g} \mathrm{kg}^{-1}\right)$ prepared, injected into devices and calibration graphs are drawn. 


$$
-6891 \text { - }
$$

\section{Precision}

\section{Repeatability}

For repeatability study; A total of 40 recovery studies were performed by 4 analysts for GC-MS at a concentration of $20 \mu \mathrm{g} \mathrm{kg}^{-1}$ and $100 \mu \mathrm{g} \mathrm{kg}^{-1}$, for LC-MS/MS at concentrations of $10 \mu \mathrm{g} \mathrm{kg}^{-1}$ and $100 \mu \mathrm{g} \mathrm{kg}^{-1}$, each with 5 replicates.

For repeatability control, RSD\% values were calculated for each person and for each concentration separately. For each individual and for each concentration, the RSD values were calculated from the mean $(\bar{x})$ and standard deviation $(\mathrm{s})$ of the measured concentrations, separately. Then the $\mathrm{RSD}_{\text {pool }}$ values were calculated and the repeatability control was performed: $\mathrm{RSD}=\frac{a}{\bar{x}}$.

\section{Reproducibility}

Intra-laboratory reproducibility study; $20 \mu \mathrm{g} \mathrm{kg}{ }^{-1}$ and $100 \mu \mathrm{g} \mathrm{kg}^{-1}$ for GC-MS, LC-MS/ MS at concentrations of $10 \mu \mathrm{g} \mathrm{kg}^{-1}$ and $100 \mu \mathrm{g} \mathrm{kg}^{-1}$, by 4 analysts for each at five different times, recovery work, was carried out. For each concentration, RSD $_{\mathrm{WR}}$ values were calculated and reproducibility checked.

\section{Accuracy}

Reality study for accuracy was done with recovery. For the reality parameter; A total of 40 recovery studies were carried out by 4 analysts at concentrations of $20 \mu \mathrm{g} \mathrm{kg}^{-1}$ and $100 \mu \mathrm{g} \mathrm{kg}^{-1}$ for GC-MS $10 \mu \mathrm{g} \mathrm{kg}^{-1}$ and $100 \mu \mathrm{g} \mathrm{kg}^{-1}$ for LC-MS/MS each. For reality control, all recovery values must be in the range of $70-120 \%$.

Percentage recovery $(\% \mathrm{R})$ values were calculated for each individual, each concentration and each repetition. $\% \mathrm{R}=\frac{\left(\mathrm{c} 2-\mathrm{Cl}_{1}\right)}{\mathrm{c}^{3}} .100, \mathrm{Cl}$ : Measured sample concentration (blank), C2: Measured, spike made sample concentration (blank + spike), C3: Spike made concentration.

\section{Uncertainty assessment}

The standard uncertainty (ux) from reality is equal to the standard deviation $\left(\mathrm{S}_{\overline{\mathrm{x}}}\right)$ of the mean, if the unit average recovery value $(\overline{\mathrm{R}})$ is not significantly different from $1 . \mathrm{t}$ test değer was applied to determine if the unit mean recovery value was significantly different from 1.

- If $\mathrm{t}<\mathrm{t}_{\text {table, }}$ then the reality standard uncertainty (ux) is equal to the, mean deviation $\left(S_{\bar{x}}\right)$ of the mean.

- If $t \geq t_{\text {table, }}$, the elevated standard uncertainty (ui) value should be calculated.

$$
u i=\sqrt{(\Pi[1-\overline{\mathrm{R}}] / k])^{2}+\left(u_{w}\right)^{2}}
$$

where

$\mathrm{k}$ : Scope factor $(\mathrm{k}=2$, for $95 \%$ confidence interval)

$\bar{R}$ : Unit average recovery value.

$$
\overline{\mathrm{R}}=\frac{\overline{\% \mathrm{R}}}{100}
$$




$$
\begin{gathered}
S_{\bar{x}}=\frac{s}{\sqrt{n}} \\
\mathrm{t}=\frac{\mid 1-\overline{\mathbb{R}}}{S_{\bar{x}}}
\end{gathered}
$$

where

$S_{\bar{x}}:$ Standard deviation of mean

$\mathrm{s}:$ Standard deviation

$\mathrm{n}$ : Total concentrations and total number of repetitions for all contacts.

In cucumber and grapefruit, the standard uncertainty from reality and the relative standard uncertainty were calculated using the Equations 1, 2, 3 and 4.

$$
R S D_{\text {pool }}=\sqrt{\frac{\left(n_{1}-1\right) x E S D_{1}^{2}+\left(n_{3}-1\right) x R S D_{2}^{2}+\cdots+\left(n_{n}-1\right) x R S D_{n}^{2}}{\left(n_{1}-1\right)+\left(n_{2}-1\right)+\cdots+\left(n_{n}-1\right)}}
$$

In cucumber and grapefruit, relative standard uncertainty from repeatability was calculated using Equation 5.

$$
R S D W R(p o w)=\sqrt{\frac{\left.C_{1}-1\right) x R S D_{W R}^{n}+\left(n_{2}-1\right) x R S D_{W R 2}^{2}}{\left(n_{1}-1\right)+\left(n_{2}-1\right)}}
$$

In cucumber and grapefruit, standard uncertainty from reproducibility was calculated using Equation 6. Relative standard uncertainty values are used in the total combined uncertainty calculation; however, since the relative standard deviation values are already used in the reproducibility calculations as in the reproducibility uncertainty calculations, the calculated standard uncertainty value is also equal to the relative standard uncertainty value.

\section{Results and discussion}

\section{Method validation and application}

Selectivity was evaluated by checking the presence of coextracted interferences in the chromatograms from blank samples. Hence, chromatograms of blank and fortificated $(100 \mu \mathrm{g} / \mathrm{kg})$ samples of cucumber and grapefruit were compared (Figure 1). The presence of matrix interferences were interpreted by monitoring the SIM chromatograms for each pesticide at the retention time window expected for each compound. It can be observed that there are no extracted matrix interferences for the determination of the pesticides studied in both fruits; therefore this method can be considered as selective.

The matrix compatible standard calibration curves showed smooth linearity over a concentration range of 20- $100 \mu \mathrm{g} \mathrm{kg}^{-1}$. As shown in Figure 2, correlation coefficients were $>0.98$. The values of Limits of detection (LOD) ranged from 0.17 to $11.8 \mu \mathrm{g} \mathrm{kg}^{-1}$ and Limits of quantification (LOQ) ranged from 0.42 to $39.35 \mu \mathrm{g} \mathrm{kg}^{-1}$ in cucumber, whereas for grapefruit, LOD, and LOQ values varied from $0.13 \mu \mathrm{g} \mathrm{kg}^{-1}$ to $11.80 \mu \mathrm{g} \mathrm{kg}^{-}$ ${ }^{1}, 0.42 \mu \mathrm{g} \mathrm{kg}^{-1}$, and $39.47 \mu \mathrm{g} \mathrm{kg}{ }^{-1}$, respectively.

The baseline (basal signal) in blank samples at the time window in which each pesticide is expected is different due to the quantification being made using different 
ions. Therefore, the LOD and LOQ calculation are different for each compound. With these results it can be concluded that the method has good sensibility for cucumber and grapefruit fruits because LOD and LOQ values of all pesticides are $<0.50 \mathrm{mg} / \mathrm{kg}$. The LOQ were well below the maximum residue levels (MRL) established by European Union for regulated pesticide residues both in cucumber and grapefruit, except for methylparathion, malathion and diazinon.

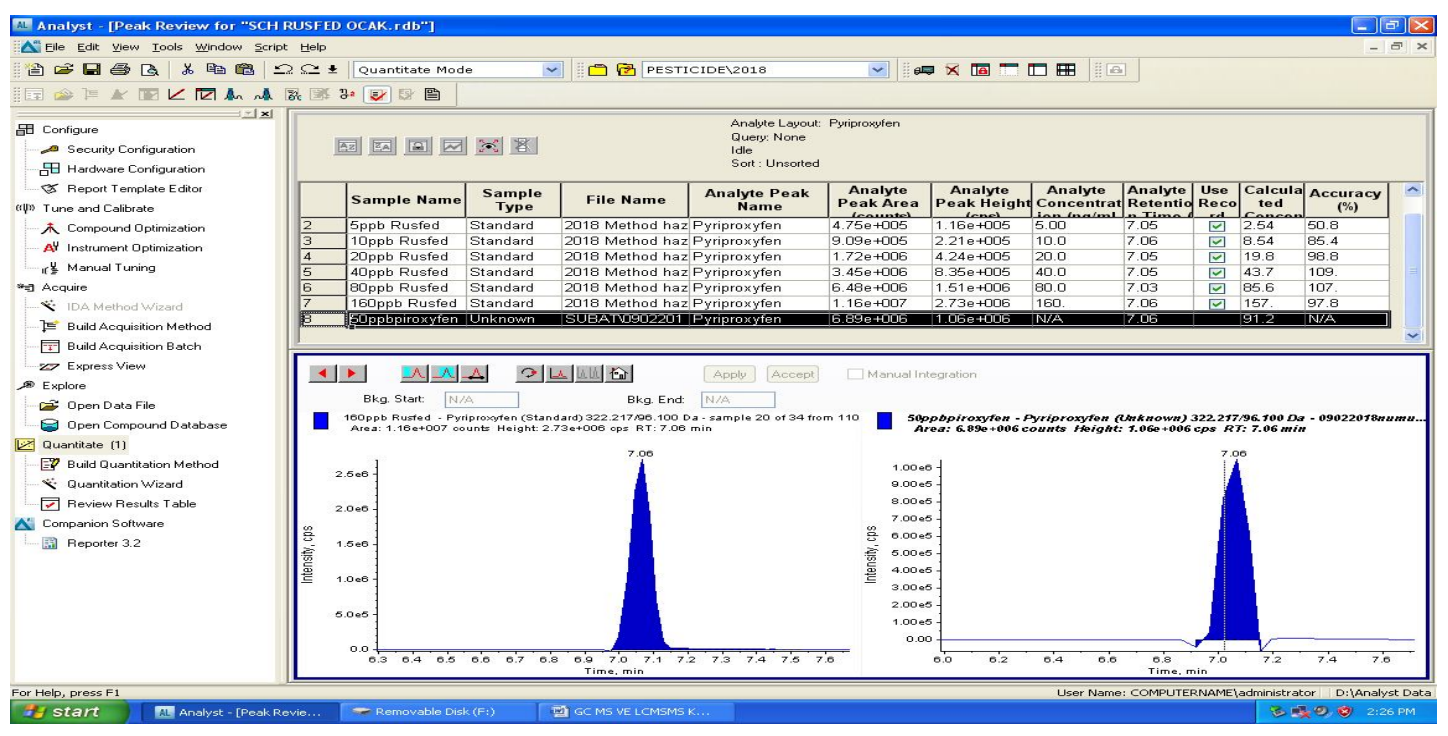

Figure 1. Chromatograms (SIM mode) obtained for pyrproxyfen injection of blank and fortified samples of cucumber and grapefruit for checking matrix interferences. Pesticide concentration was $100 \mu \mathrm{g} / \mathrm{L}$

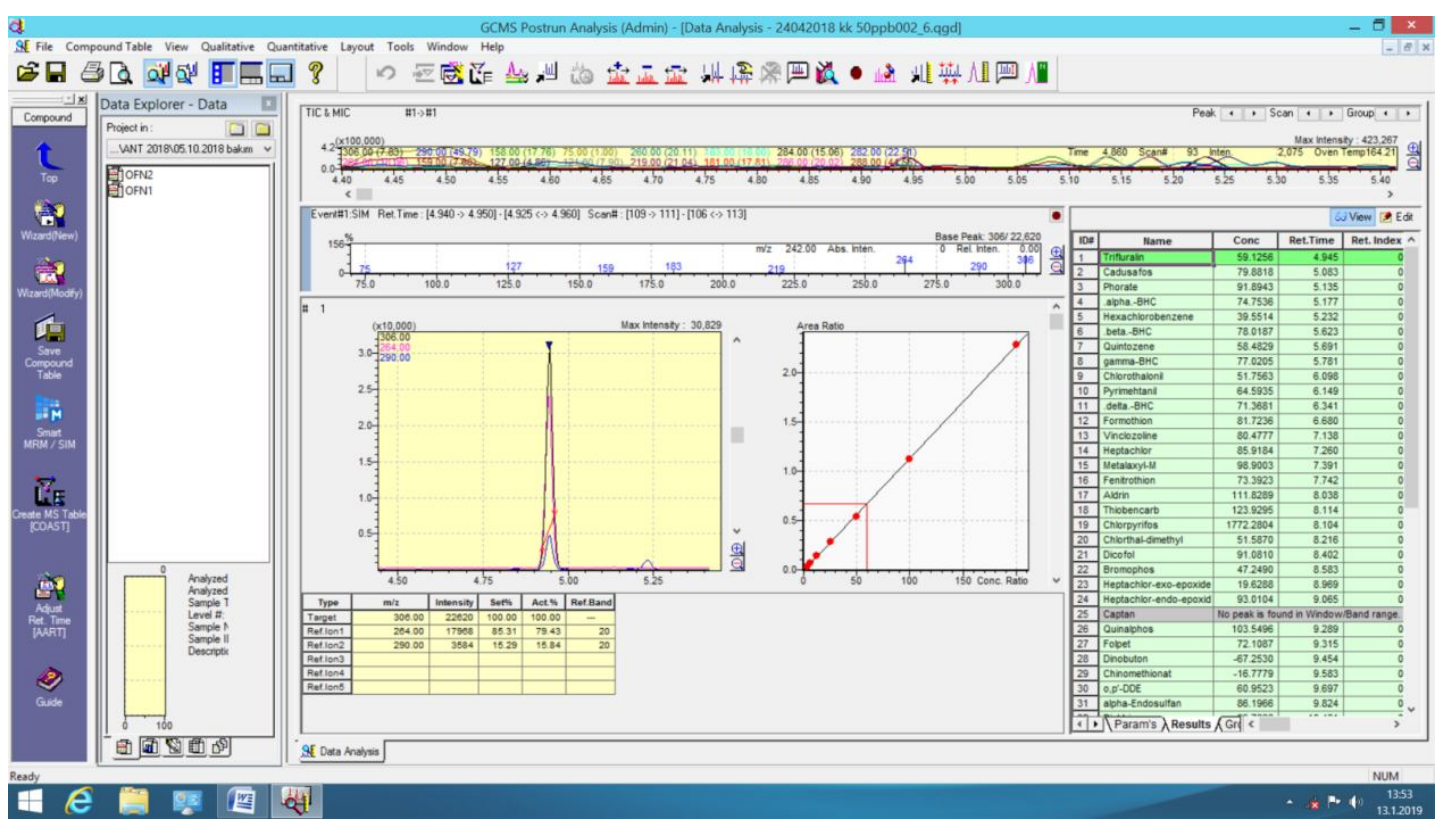

Figure 2. Chromatograms (SIM mode) obtained for trifluralin injection of blank and fortified samples of cucumber and grapefruit for checking matrix interferences. Pesticide concentration was $100 \mu \mathrm{g} / \mathrm{L}$ 
The recovery values correspond to the lowest and highest concentration of the linear range. The recovery results are as shown in Table 1. Recoveries obtained for each pesticide in both matrices ranged between $77.87 \%$ and $104.15 \%$. Thus, the recovery data is in the range of values required by the SANTE Guidelines, which recommends a recovery rate of $70-120 \%$ (European Commission, 2015).

Precision was expressed as relative standard deviation (RSD) and evaluated in different days (see Table 1). For this purpose, $20 \mu \mathrm{g} \mathrm{kg}^{-1}$ and $100 \mu \mathrm{g} \mathrm{kg}^{-1}$ for GC-MS, LC-MS / MS at concentrations of $10 \mu \mathrm{g} \mathrm{kg}^{-1}$ and $100 \mu \mathrm{g} \mathrm{kg}^{-1}$, by four analysts for each at five different times, recovery work, was carried out. Relative standard deviation (RSD) values calculated were $>20 \%(24.32 \%)$ for clofentazine in cucumber at $20.0 \mathrm{mg}$ $\mathrm{kg}^{-1}$ and $(21.36 \%)$ for propyzamide, $(29.11 \%)$ for fludioxonil grapefruit at $100 \mu \mathrm{g} \mathrm{kg}^{-1}$. For the other compounds, at both concentration levels evaluated in cucumber and grapefruit, the RSD values were $<20 \%$. The RSD values obtained are considered satisfactory according to the European SANTE Guideline (European Commission, 2015).

\section{Pesticide analysis in commercial fruit and vegetable samples}

The validated analytical method was used to analyse 233 pesticide residues in cucumber and grapefruit samples collected from bazaar, supermarkets in four provinces of Turkey. In our monitoring program, a total of 680 vegetable samples, including 75 cucumbers and 110 grapefruits in 2015, 90 cucumbers and 120 grapefruits in 2016, and 165 cucumbers and 120 grapefruits in 2017, were analysed. Of the 680 fruit and vegetable samples, $11.6 \%$ (35 samples) of cucumbers and 13.8\% (39 samples) of grapefruits contained at least one detectable residue, but levels were below the EU MRL. There were 1 acaricide, 3 insecticides and 5 fungicides in cucumbers, while 4 insecticides and 4 fungicides were detected in grapefruit samples.

While 27 cucumber samples gave positive results, multiple pesticide residues were found in 8 samples, corresponding to $2.4 \%$ of the total samples. Clofentezine was the most frequently detected pesticide measured in cucember with a percentage occurrence value of $4.4 \%$, and in concentrations ranging from 0.021 to $0.042 \mathrm{mg} \mathrm{kg}$. This insecticide is an acaricide used in various products (fruit crops, leafy vegetables, some fruiting vegetables, citrus fruits, pome fruits, grapes, cotton etc.) against red spider species. Acaricides in this group are contact effective. They also have a gastric venom effect (European Commission, 2008; Van Leeuwen et al., 2010).

The fungicide propyzamide was the second most abundant pesticide (199 out of 330 samples) in cucumbers, with levels ranging from 0.002 to $0.009 \mathrm{mg} \mathrm{kg}^{-1}$. Propyzamide, effectively controls a wide range of herbicides in several agricultural crops, including hard-shelled fruits, in grape fruits, and tree nuts. It has a chronic toxic effect (European Commission, 2008; Tian et al., 2018).

The fungicide propamocarb was the third most abundant pesticide (230 out of 330 samples) in cucumbers, with levels ranging from 0.022 to $0.032 \mathrm{mg} \mathrm{kg}^{-1}$. Propamocarb, which belongs to the class of carbamate, is used in cucumber against mildew. Does not have toxic effects (FAO, 2012; Bhat et al., 2018).

Azinphosethyl was detected individually in 6 cucumber samples (1.8\%) in concentrations between 0.013 and $0.016 \mathrm{mg} \mathrm{kg}^{-1}$. It has a protective and therapeutic properties. Alternaria, Botrytis, Pythium, Rhizoctonia, Venturia and many other diseases are widely used as a fungicide. It has no acute and chronic effects (Polyiem et al., 2018). 


$$
-6895-
$$

Table 1. LOD, LOQ study results for GC-MS and LC-MS / MS

\begin{tabular}{|c|c|c|c|c|c|c|c|c|c|c|c|c|}
\hline \multirow{5}{*}{ Pesticides } & \multicolumn{6}{|c|}{ Cucumber } & \multicolumn{6}{|c|}{ Grapefruit } \\
\hline & \multirow{4}{*}{ LOD } & \multirow{4}{*}{ LOQ } & \multirow{2}{*}{\multicolumn{2}{|c|}{$\begin{array}{l}\text { Precision } \\
\text { RSD (\%) }\end{array}$}} & \multirow{2}{*}{\multicolumn{2}{|c|}{$\frac{\text { Recovery }}{(\%)}$}} & \multirow{4}{*}{ LOD } & \multirow{4}{*}{ LOQ } & \multirow{2}{*}{\multicolumn{2}{|c|}{$\begin{array}{l}\text { Precision } \\
\text { RSD (\%) }\end{array}$}} & \multirow{2}{*}{\multicolumn{2}{|c|}{$\frac{\text { Recovery }}{(\%)}$}} \\
\hline & & & & & & & & & & & & \\
\hline & & & 20 & 100 & 20 & 100 & & & 10 & 100 & 10 & 100 \\
\hline & & & \multicolumn{4}{|c|}{$(\mu \mathrm{g} / \mathrm{kg})$} & & & \multicolumn{4}{|c|}{$(\mu \mathrm{g} / \mathrm{kg})$} \\
\hline Trifluralin & 8.08 & 26.94 & 7.45 & 8.65 & 101.91 & 88.39 & 1.04 & 19.26 & 9.15 & 12.54 & 101.91 & 87.45 \\
\hline Chlorothalonil & 4.78 & 15.94 & 8.65 & 7.23 & 100.96 & 85.72 & 2.03 & 17.28 & 9.12 & 14.56 & 94.87 & 100.69 \\
\hline Formothion & 6.37 & 21.25 & 9.21 & 6.14 & 96.5 & 88.88 & 2.96 & 17.14 & 10.12 & 17.85 & 95.67 & 91.24 \\
\hline Vinclozolin & 5.76 & 19.2 & 11.45 & 8.74 & 104.15 & 91.82 & 2.38 & 10.89 & 15.14 & 19.63 & 104.15 & 96.78 \\
\hline Fenitrothion & 8.27 & 27.58 & 10.54 & 9.65 & 100.6 & 88.4 & 4.99 & 5.98 & 14.12 & 15.36 & 100.6 & 85.92 \\
\hline Dicofol & 5.66 & 18.87 & 9.65 & 7.32 & 103.01 & 92.15 & 3.85 & 10.66 & 11.1 & 19.36 & 103.01 & 88.41 \\
\hline Captan & 7.31 & 24.38 & 13.65 & 7.65 & 102.61 & 83.91 & 4.53 & 10.14 & 13.1 & 18.36 & 102.61 & 90.69 \\
\hline Folpet & 6.8 & 22.67 & 18.65 & 6.84 & 93.95 & 88.28 & 3.65 & 8.52 & 10.21 & 10.15 & 95.62 & 87.3 \\
\hline Qinomethionate & 10.15 & 33.83 & 11.32 & 6.45 & 99.51 & 91.2 & 3.97 & 5.03 & 10.14 & 17.32 & 93.73 & 103.21 \\
\hline Dinobuton & 11.8 & 39.35 & 9.32 & 6.21 & 98.75 & 89.45 & 5.26 & 15.42 & 9.85 & 18.36 & 94.54 & 92.38 \\
\hline Endosulfan A & 7.56 & 25.2 & 8.65 & 7.52 & 99.77 & 88.52 & 3.64 & 6.89 & 7.65 & 11.1 & 99.77 & 99.56 \\
\hline Endosulfan B & 7.38 & 24.61 & 7.45 & 6.36 & 101.08 & 89.17 & 2.99 & 3.48 & 12.69 & 11.2 & 101.08 & 91.18 \\
\hline Bromopropylate & 7.49 & 24.96 & 14.12 & 15.36 & 102.61 & 78.55 & 4.77 & 6.77 & 11.36 & 10.21 & 102.61 & 88.69 \\
\hline Tetradifon & 6.88 & 22.93 & 15.36 & 19.36 & 102.27 & 89.25 & 2.57 & 9.86 & 10.87 & 9.65 & 102.27 & 93.87 \\
\hline Alfa-Cypermethrin a & 7.38 & 24.58 & 18.69 & 11.36 & 102.82 & 90.64 & 3.76 & 7.93 & 10.96 & 8.65 & 102.82 & 86.17 \\
\hline Alfa-Cypermethrin b & 7.2 & 24.02 & 14.65 & 10.15 & 97.51 & 91.77 & 4.71 & 16.63 & 10.23 & 9.67 & 92.82 & 99.31 \\
\hline alpha.-BHC & 3.96 & 13.19 & 15.21 & 17.32 & 98.26 & 91.28 & 3.02 & 12.82 & 10.65 & 8.63 & 98.99 & 97.52 \\
\hline delta.-BHC & 5.94 & 19.79 & 17.45 & 18.36 & 99.6 & 88 & 2.46 & 15.11 & 11.23 & 8.65 & 99.6 & 90.54 \\
\hline Heptachlor & 7.2 & 23.99 & 18.21 & 11.1 & 101.72 & 80.22 & 4.83 & 12.16 & 12.32 & 9.45 & 101.72 & 90.64 \\
\hline 2,4-DDE & 4.45 & 14.85 & 19.32 & 11.2 & 101.45 & 83.73 & 5.13 & 13.23 & 11.56 & 14.65 & 101.45 & 100.53 \\
\hline Dieldrin & 4.24 & 14.13 & 18.65 & 10.54 & 98.88 & 83.67 & 5.54 & 17.53 & 11.45 & 19.32 & 98.88 & 90.56 \\
\hline 4,4-DDE & 5.78 & 19.26 & 15.32 & 14.32 & 101.79 & 94.44 & 3.76 & 12.14 & 10.65 & 18.65 & 101.79 & 89.22 \\
\hline 2,4-DDD & 4.8 & 16.01 & 11.35 & 12.36 & 99.8 & 88.56 & 4.32 & 9.97 & 9.63 & 15.32 & 99.8 & 93.71 \\
\hline Endrin & 3.49 & 11.64 & 9.14 & 10.23 & 102.21 & 93.36 & 6.01 & 15.89 & 7.23 & 11.35 & 102.21 & 99.19 \\
\hline Chlorfenapyl & 5.82 & 19.4 & 8.54 & 17.36 & 100.93 & 90.78 & 4.44 & 8.56 & 9.63 & 9.14 & 100.93 & 93.53 \\
\hline 4,4-DDD & 4.84 & 16.12 & 11.21 & 14.65 & 102.11 & 95.72 & 4.88 & 12.54 & 10.25 & 8.54 & 102.11 & 99.48 \\
\hline Ethion & 8.6 & 28.67 & 12.41 & 13.85 & 101.54 & 87.48 & 3.07 & 15.69 & 14.52 & 11.21 & 101.54 & 91.5 \\
\hline DDT & 6.69 & 22.31 & 16.74 & 14.87 & 102.37 & 91.95 & 4.75 & 10.05 & 11.52 & 12.41 & 102.37 & 98.37 \\
\hline Abamectin & 4.07 & 13.56 & 18.69 & 14.23 & 87.45 & 89.17 & 4.12 & 8.21 & 10.65 & 16.74 & 88.39 & 92.89 \\
\hline Acetamiprid & 4.32 & 14.41 & 17.21 & 16.65 & 100.69 & 91.6 & 2.87 & 16.09 & 10.65 & 18.69 & 85.72 & 90.59 \\
\hline Acetochlor & 4.2 & 14.01 & 11.32 & 18.98 & 91.24 & 88.53 & 2.42 & 17.09 & 11.23 & 17.21 & 88.88 & 91.85 \\
\hline Acrinathrin & 4.75 & 15.82 & 8.14 & 9.47 & 96.78 & 89.55 & 0.49 & 18.45 & 11.25 & 14.32 & 91.82 & 90.79 \\
\hline Alachlor & 5.04 & 16.79 & 7.65 & 8.31 & 85.92 & 89.36 & 3.01 & 12.54 & 10.87 & 12.63 & 88.4 & 91.43 \\
\hline Amitraz & 3.23 & 10.75 & 10.14 & 7.54 & 88.41 & 86.21 & 5.85 & 14.41 & 9.63 & 14.36 & 92.15 & 90.31 \\
\hline Atrazine & 2.43 & 8.1 & 14.65 & 7.98 & 90.69 & 93.05 & 3.26 & 20.03 & 8.35 & 12.31 & 83.91 & 94.33 \\
\hline Azinphos methyl & 5.01 & 16.68 & 14.12 & 7.47 & 87.3 & 89.89 & 2.4 & 14.81 & 8.65 & 11.32 & 88.28 & 90.22 \\
\hline Azoxystrobine & 6.17 & 20.58 & 11.25 & 6.32 & 103.21 & 89.93 & 4.97 & 16.27 & 7.65 & 10.63 & 91.2 & 104.09 \\
\hline Bensulfuron-Methyl & 0.13 & 0.42 & 17.32 & 14.36 & 92.38 & 95.37 & 4.14 & 10.24 & 8.32 & 9.36 & 89.45 & 94.94 \\
\hline Bentazone & 3.09 & 10.3 & 18.36 & 12.31 & 99.56 & 90.67 & 4.42 & 15.84 & 9.63 & 9.56 & 88.52 & 91.4 \\
\hline Beta-Cyfluthrin & 3.39 & 11.3 & 11.1 & 11.32 & 91.18 & 91.11 & 3.51 & 13.73 & 12.54 & 8.36 & 89.17 & 89.6 \\
\hline
\end{tabular}




$$
-6896-
$$

\begin{tabular}{|c|c|c|c|c|c|c|c|c|c|c|c|c|}
\hline \multirow{5}{*}{ Pesticides } & \multicolumn{6}{|c|}{ Cucumber } & \multicolumn{6}{|c|}{ Grapefruit } \\
\hline & \multirow{4}{*}{ LOD } & \multirow{4}{*}{ LOQ } & \multirow{2}{*}{\multicolumn{2}{|c|}{$\begin{array}{l}\text { Precision } \\
\text { RSD (\%) }\end{array}$}} & \multirow{2}{*}{\multicolumn{2}{|c|}{$\frac{\text { Recovery }}{(\%)}$}} & \multirow{4}{*}{ LOD } & \multirow{4}{*}{ LOQ } & \multirow{2}{*}{\multicolumn{2}{|c|}{$\begin{array}{c}\text { Precision } \\
\operatorname{RSD}(\%)\end{array}$}} & \multirow{2}{*}{\multicolumn{2}{|c|}{$\frac{\text { Recovery }}{(\%)}$}} \\
\hline & & & & & & & & & & & & \\
\hline & & & 20 & 100 & 20 & 100 & & & 10 & 100 & 10 & 100 \\
\hline & & & \multicolumn{4}{|c|}{$(\mu \mathrm{g} / \mathrm{kg})$} & & & \multicolumn{4}{|c|}{$(\mu \mathrm{g} / \mathrm{kg})$} \\
\hline Bifenthrin & 2.09 & 6.96 & 11.2 & 10.63 & 88.69 & 86.67 & 5.55 & 9.57 & 14.56 & 9.1 & 78.55 & 98.32 \\
\hline Boscalid & 4.23 & 14.1 & 10.21 & 9.36 & 93.87 & 88.36 & 5.94 & 8.08 & 17.85 & 9.89 & 89.25 & 92.43 \\
\hline Bromoxynil & 2.68 & 8.92 & 9.65 & 9.56 & 86.17 & 87.84 & 3.3 & 1.63 & 19.63 & 8.63 & 90.64 & 87.48 \\
\hline Bromuconazole & 4.18 & 13.92 & 8.65 & 8.36 & 99.31 & 90.22 & 4.69 & 10.02 & 15.36 & 9.52 & 91.77 & 92.72 \\
\hline Bupirimate & 5.81 & 19.37 & 9.67 & 9.1 & 97.52 & 87.81 & 3.6 & 19.5 & 19.36 & 9.38 & 91.28 & 87.53 \\
\hline Buprofezine & 3.56 & 11.87 & 8.63 & 9.89 & 90.54 & 88.94 & 4.66 & 10.86 & 11.36 & 10.54 & 88 & 89.81 \\
\hline Carbaryl & 1.8 & 5.99 & 8.65 & 14.65 & 90.64 & 88 & 5.16 & 8.45 & 18.65 & 11.52 & 80.22 & 86.85 \\
\hline Carbendazim & 1.24 & 4.12 & 9.45 & 12.32 & 100.53 & 93.49 & 4.08 & 16.56 & 11.32 & 7.65 & 83.73 & 83.68 \\
\hline Carbofuran & 2.33 & 7.76 & 14.65 & 17.14 & 90.56 & 90.44 & 4.13 & 13.8 & 9.32 & 12.69 & 83.67 & 94.06 \\
\hline Carbosulfan & 3.65 & 12.16 & 17.23 & 11.65 & 89.22 & 87.56 & 5.98 & 14.75 & 8.65 & 11.36 & 94.44 & 91.79 \\
\hline Carboxin & 2.43 & 8.11 & 15.21 & 12.31 & 93.71 & 84.98 & 1.34 & 11.69 & 7.45 & 10.87 & 88.56 & 91.78 \\
\hline Carfentrazone-Ethyl & 4.49 & 14.97 & 14.32 & 11.32 & 99.19 & 89.06 & 3.32 & 18.51 & 14.12 & 10.96 & 93.36 & 99.05 \\
\hline Chlofentazine & 4.58 & 15.28 & 24.32 & 10.63 & 93.53 & 95.76 & 1.27 & 19.81 & 15.36 & 10.23 & 90.78 & 92.5 \\
\hline Chlorfluozuron & 2.83 & 9.44 & 14.36 & 9.36 & 99.48 & 87.17 & 1.64 & 11.01 & 18.69 & 10.65 & 95.72 & 84.8 \\
\hline Chloridazon & 4.01 & 13.37 & 12.31 & 9.56 & 91.5 & 96.47 & 4.34 & 15.65 & 14.65 & 11.23 & 87.48 & 95.69 \\
\hline $\begin{array}{l}\text { Chlormequat } \\
\text { chloride }\end{array}$ & 0.65 & 2.17 & 11.32 & 8.36 & 98.37 & 92.06 & 4.99 & 12.14 & 15.21 & 12.32 & 91.95 & 102.17 \\
\hline Chlorpham & 4.95 & 16.51 & 10.63 & 9.1 & 92.89 & 96.36 & 3.29 & 15.54 & 17.45 & 11.56 & 89.17 & 92.83 \\
\hline Chlorpyrifos ethyl & 5.11 & 17.02 & 9.36 & 9.89 & 90.59 & 92.62 & 1.48 & 17.19 & 18.21 & 11.45 & 91.6 & 95.66 \\
\hline $\begin{array}{l}\text { Chlorpyriphos } \\
\text { Methyl }\end{array}$ & 5.82 & 19.41 & 9.56 & 8.63 & 91.85 & 86.75 & 3.46 & 13.59 & 19.32 & 9.45 & 88.53 & 88.12 \\
\hline Chlorsulfuron & 4.9 & 16.33 & 8.36 & 9.52 & 90.79 & 88.56 & 1.27 & 13.77 & 18.65 & 14.65 & 89.55 & 90.98 \\
\hline Clethodim & 2.76 & 9.2 & 9.1 & 10.65 & 91.43 & 89.94 & 4.77 & 19.93 & 15.32 & 17.23 & 89.36 & 90.15 \\
\hline $\begin{array}{l}\text { Clodinafop- } \\
\text { Propargyl }\end{array}$ & 4.97 & 16.57 & 9.89 & 8.36 & 90.31 & 86.66 & 1.28 & 4.47 & 11.35 & 15.21 & 86.21 & 93.05 \\
\hline Clothianidin & 2.74 & 9.12 & 8.63 & 9.1 & 94.33 & 92.59 & 5.93 & 11.07 & 9.14 & 14.32 & 93.05 & 98.78 \\
\hline Cyclanilide & 4.61 & 15.36 & 9.52 & 9.89 & 90.22 & 91.03 & 4.48 & 4.23 & 8.54 & 12.63 & 89.89 & 95.08 \\
\hline Cycloate & 3.73 & 12.44 & 9.38 & 8.63 & 104.09 & 90.99 & 4.99 & 5.46 & 11.21 & 14.36 & 89.93 & 90.12 \\
\hline Cyhalofop-butyl & 3.01 & 10.03 & 10.54 & 9.52 & 94.94 & 97.87 & 4.38 & 14.48 & 12.41 & 12.31 & 95.37 & 90.62 \\
\hline Cymoxanyl & 0.52 & 1.72 & 11.52 & 9.38 & 91.4 & 85.36 & 5.99 & 16.63 & 10.63 & 11.32 & 90.67 & 90.67 \\
\hline Cypermethrin & 2.17 & 7.22 & 10.45 & 10.54 & 89.6 & 82.92 & 5.89 & 10.95 & 9.36 & 5.12 & 91.11 & 88.33 \\
\hline Cyproconazole & 5.36 & 17.85 & 10.78 & 11.52 & 98.32 & 86.39 & 6.36 & 4.93 & 9.56 & 4.12 & 86.67 & 87.89 \\
\hline Cyprodinile & 4.67 & 15.57 & 11.65 & 7.65 & 92.43 & 89.93 & 2.38 & 11.53 & 8.36 & 6.18 & 88.36 & 90.05 \\
\hline Dazomet & 1.09 & 3.62 & 5.65 & 12.69 & 87.48 & 88.64 & 4.55 & 4.23 & 9.17 & 6.45 & 87.84 & 99.29 \\
\hline Deltamethrin & 3.95 & 13.16 & 6.85 & 14.36 & 92.72 & 88.57 & 4.03 & 15.91 & 9.89 & 8.12 & 90.22 & 96.14 \\
\hline Diafenthiuron & 3.4 & 11.32 & 7.65 & 12.31 & 87.53 & 86.73 & 4.22 & 4.28 & 8.63 & 9.15 & 87.81 & 94.59 \\
\hline Diazinon & 3.46 & 11.53 & 8.41 & 11.32 & 89.81 & 85.88 & 6.06 & 19.77 & 9.52 & 9.12 & 88.94 & 95.27 \\
\hline Dicamba & 3.51 & 11.71 & 8.69 & 10.63 & 86.85 & 88.28 & 2.13 & 14.95 & 9.38 & 10.12 & 88 & 96.27 \\
\hline Dichlofluanid & 2.08 & 6.94 & 7.36 & 9.36 & 83.68 & 88.85 & 4.97 & 16.62 & 10.54 & 15.14 & 93.49 & 89.8 \\
\hline Dichlorvos & 3.21 & 10.71 & 8.65 & 9.56 & 94.06 & 93.14 & 4.81 & 14.59 & 11.52 & 14.12 & 90.44 & 100.03 \\
\hline Diclofop Methyl & 2.7 & 8.99 & 9.36 & 8.36 & 91.79 & 88.32 & 5.55 & 19.97 & 14.32 & 11.1 & 87.56 & 94.38 \\
\hline Diethofencarb & 3.67 & 12.23 & 11.52 & 9.1 & 91.78 & 93.82 & 4.28 & 19.64 & 12.63 & 13.1 & 84.98 & 97.31 \\
\hline Difenoconazole & 4.05 & 13.49 & 10.54 & 9.89 & 99.05 & 92.41 & 3.96 & 21.21 & 14.36 & 14.12 & 89.06 & 93.77 \\
\hline Diflubenzuron & 3.53 & 11.78 & 11.57 & 14.65 & 92.5 & 91.96 & 5.7 & 7.92 & 12.31 & 17.52 & 95.76 & 94.41 \\
\hline
\end{tabular}




$$
-6897 \text { - }
$$

\begin{tabular}{|c|c|c|c|c|c|c|c|c|c|c|c|c|}
\hline \multirow{5}{*}{ Pesticides } & \multicolumn{6}{|c|}{ Cucumber } & \multicolumn{6}{|c|}{ Grapefruit } \\
\hline & \multirow{4}{*}{ LOD } & \multirow{4}{*}{ LOQ } & \multirow{2}{*}{\multicolumn{2}{|c|}{$\begin{array}{l}\text { Precision } \\
\text { RSD (\%) }\end{array}$}} & \multirow{2}{*}{\multicolumn{2}{|c|}{$\frac{\text { Recovery }}{(\%)}$}} & \multirow{4}{*}{ LOD } & \multirow{4}{*}{ LOQ } & \multirow{2}{*}{\multicolumn{2}{|c|}{$\begin{array}{c}\text { Precision } \\
\text { RSD (\%) }\end{array}$}} & \multirow{2}{*}{\multicolumn{2}{|c|}{$\frac{\text { Recovery }}{(\%)}$}} \\
\hline & & & & & & & & & & & & \\
\hline & & & 20 & 100 & 20 & 100 & & & 10 & 100 & 10 & 100 \\
\hline & & & \multicolumn{4}{|c|}{$(\mu \mathrm{g} / \mathrm{kg})$} & & & \multicolumn{4}{|c|}{$(\mu \mathrm{g} / \mathrm{kg})$} \\
\hline Dimethenamid & 4.68 & 15.6 & 12.65 & 12.32 & 84.8 & 93.18 & 4.37 & 15.17 & 11.32 & 10.32 & 87.17 & 92.01 \\
\hline Dimethoate & 3.02 & 10.05 & 13.25 & 15.32 & 95.69 & 90.99 & 5.82 & 13.43 & 10.63 & 9.32 & 96.47 & 90.12 \\
\hline Dimethomorph & 4.6 & 15.35 & 14.32 & 10.11 & 102.17 & 91.82 & 0.17 & 14.08 & 9.36 & 10.65 & 92.06 & 98.19 \\
\hline Diniconazole & 4.83 & 16.11 & 14.58 & 11.21 & 92.83 & 83.81 & 5.17 & 20.19 & 9.56 & 11.25 & 96.36 & 86.9 \\
\hline Dinocap & 3.06 & 10.2 & 12.69 & 12.32 & 95.66 & 88.55 & 4.21 & 7.12 & 8.36 & 10.87 & 92.62 & 95.16 \\
\hline Dioxathion & 5.62 & 18.73 & 11.36 & 15.36 & 88.12 & 94.05 & 6.02 & 16.57 & 9.14 & 9.63 & 86.75 & 90.98 \\
\hline Diphenamid & 3.88 & 12.93 & 10.87 & 19.36 & 90.98 & 92.6 & 5.82 & 16.05 & 9.89 & 8.35 & 88.56 & 100.65 \\
\hline Dithianon & 3.19 & 10.63 & 10.96 & 21.36 & 90.15 & 95.55 & 5.75 & 18.48 & 8.63 & 8.65 & 89.94 & 91.63 \\
\hline Diuron & 3.49 & 11.64 & 10.23 & 18.65 & 93.05 & 84.51 & 5.22 & 14.26 & 9.52 & 7.65 & 86.66 & 91.9 \\
\hline Epoxyconazole & 6.3 & 20.99 & 10.65 & 11.32 & 98.78 & 91.54 & 4.57 & 13.21 & 9.38 & 8.32 & 92.59 & 96.78 \\
\hline EPTC & 4.33 & 14.42 & 11.23 & 9.32 & 95.08 & 86.61 & 4.99 & 19.01 & 10.54 & 9.63 & 91.03 & 91.34 \\
\hline Esfenvalerate & 2.96 & 9.86 & 12.32 & 8.65 & 90.12 & 85.69 & 0.55 & 14.56 & 11.52 & 12.54 & 90.99 & 83.28 \\
\hline Ethalfluralin & 3.7 & 12.33 & 11.56 & 7.45 & 90.62 & 82.77 & 1.25 & 19.41 & 9.45 & 14.56 & 97.87 & 92.14 \\
\hline Ethiofencarb & 1.4 & 4.67 & 11.45 & 14.12 & 90.67 & 84.53 & 2.33 & 0.56 & 8.65 & 17.85 & 85.36 & 90.38 \\
\hline Ethofumasate & 4.21 & 14.03 & 10.65 & 9.32 & 88.33 & 89.26 & 4.82 & 17.23 & 7.23 & 9.74 & 82.92 & 99.63 \\
\hline Ethoprophos & 4.26 & 14.21 & 9.63 & 10.63 & 87.89 & 88.09 & 2.64 & 14.02 & 6.14 & 10.11 & 86.39 & 93.6 \\
\hline Etofenprox & 5.78 & 19.26 & 7.23 & 17.01 & 90.05 & 90.08 & 0.66 & 20.06 & 8.74 & 15.14 & 89.93 & 86.83 \\
\hline Etoxazole & 5.18 & 17.28 & 9.63 & 10.32 & 99.29 & 85.11 & 3.29 & 19.4 & 9.65 & 14.12 & 88.64 & 96.2 \\
\hline Famoxadone & 5.14 & 17.14 & 10.25 & 11.21 & 96.14 & 93.47 & 5.79 & 19.16 & 7.32 & 13.12 & 88.57 & 92.24 \\
\hline Fenamidone & 3.27 & 10.89 & 14.52 & 15.56 & 94.59 & 92.45 & 5.25 & 17.39 & 7.65 & 19.11 & 86.73 & 96.87 \\
\hline Fenamiphos (sum) & 1.79 & 5.98 & 11.52 & 9.45 & 95.27 & 91.03 & 3.91 & 15.24 & 6.84 & 18.14 & 85.88 & 97.06 \\
\hline Fenarimol & 3.2 & 10.66 & 10.65 & 14.65 & 96.27 & 86.62 & 1.69 & 16.63 & 6.45 & 17.14 & 88.28 & 93.21 \\
\hline Fenazaquin & 3.04 & 10.14 & 10.65 & 17.23 & 89.8 & 92.99 & 3.55 & 1.82 & 6.21 & 12.11 & 88.85 & 94.41 \\
\hline Fenbuconazole & 2.56 & 8.52 & 11.23 & 15.21 & 100.03 & 89.33 & 5.82 & 4.18 & 7.52 & 9.52 & 93.14 & 88.87 \\
\hline Fenoxaprop-P-Ethyl & 1.51 & 5.03 & 11.25 & 14.32 & 94.38 & 78.75 & 4.73 & 7.75 & 6.36 & 8.32 & 88.32 & 89.11 \\
\hline Fenoxycarb & 4.63 & 15.42 & 10.87 & 12.63 & 97.31 & 85.39 & 2.56 & 16.08 & 7.65 & 11.45 & 93.82 & 86.91 \\
\hline Fenpropathrin & 2.07 & 6.89 & 9.63 & 14.36 & 93.77 & 89.51 & 1.57 & 8.79 & 12.69 & 10.65 & 92.41 & 87.7 \\
\hline Fenpyroximate & 1.04 & 3.48 & 8.35 & 12.31 & 94.41 & 90.84 & 0.7 & 2.21 & 11.36 & 9.63 & 91.96 & 82.97 \\
\hline Fenthion & 2.03 & 6.77 & 8.65 & 11.32 & 92.01 & 85.23 & 0.27 & 10.96 & 10.87 & 7.23 & 93.18 & 86.94 \\
\hline Fenvalerate & 2.96 & 9.86 & 7.65 & 15.65 & 90.12 & 90.35 & 2.73 & 19.29 & 10.96 & 9.63 & 90.99 & 93.64 \\
\hline Fipronil & 2.38 & 7.93 & 8.32 & 18.69 & 98.19 & 90.9 & 4.12 & 17.51 & 10.23 & 9.52 & 91.82 & 85.09 \\
\hline Fluazifop-P-Butyl & 4.99 & 16.63 & 9.63 & 14.36 & 86.9 & 89.68 & 3.21 & 13.02 & 10.65 & 10.74 & 83.81 & 101.63 \\
\hline Fluazinam & 3.85 & 12.82 & 12.54 & 7.65 & 95.16 & 85.02 & 3.4 & 5.62 & 11.23 & 11.74 & 88.55 & 92.88 \\
\hline Fludioxynil & 4.53 & 15.11 & 14.56 & 6.84 & 90.98 & 91.03 & 1.75 & 11.82 & 12.32 & 29.11 & 94.05 & 93.48 \\
\hline Flufenoxuron & 3.65 & 12.16 & 17.85 & 6.45 & 100.65 & 94.58 & 3.24 & 19.41 & 11.56 & 17.85 & 92.6 & 94.7 \\
\hline Flurasulam & 3.97 & 13.23 & 19.63 & 6.21 & 91.63 & 92.08 & 3.59 & 15.77 & 11.45 & 19.21 & 95.55 & 92.49 \\
\hline Flurochloridone & 5.26 & 17.53 & 15.36 & 7.52 & 91.9 & 83.14 & 0.55 & 8.53 & 8.65 & 8.65 & 84.51 & 90.09 \\
\hline Flusilazole & 3.64 & 12.14 & 19.36 & 19.36 & 96.78 & 98.84 & 0.51 & 5.22 & 10.45 & 7.65 & 91.54 & 93.67 \\
\hline Flutriafol & 2.99 & 9.97 & 18.36 & 19.36 & 91.34 & 91.02 & 0.79 & 2.35 & 11.74 & 8.32 & 86.61 & 96.84 \\
\hline Foramsulfuron & 4.77 & 15.89 & 17.15 & 18.65 & 83.28 & 85.58 & 3.6 & 0.91 & 9.65 & 9.63 & 85.69 & 90.22 \\
\hline Formatanate & 2.57 & 8.56 & 19.56 & 11.32 & 92.14 & 77.87 & 4.76 & 9.09 & 10.65 & 12.54 & 82.77 & 83.83 \\
\hline Fosthiazate & 3.76 & 12.54 & 18.65 & 9.32 & 90.38 & 82.08 & 3.99 & 13.72 & 11.63 & 14.56 & 84.53 & 91.1 \\
\hline
\end{tabular}




$$
-6898 \text { - }
$$

\begin{tabular}{|c|c|c|c|c|c|c|c|c|c|c|c|c|}
\hline \multirow{5}{*}{ Pesticides } & \multicolumn{6}{|c|}{ Cucumber } & \multicolumn{6}{|c|}{ Grapefruit } \\
\hline & \multirow{4}{*}{ LOD } & \multirow{4}{*}{ LOQ } & \multirow{2}{*}{\multicolumn{2}{|c|}{$\begin{array}{l}\text { Precision } \\
\text { RSD (\%) }\end{array}$}} & \multirow{2}{*}{\multicolumn{2}{|c|}{$\frac{\text { Recovery }}{(\%)}$}} & \multirow{4}{*}{ LOD } & \multirow{4}{*}{ LOQ } & \multirow{2}{*}{\multicolumn{2}{|c|}{$\begin{array}{l}\text { Precision } \\
\text { RSD (\%) }\end{array}$}} & \multirow{2}{*}{\multicolumn{2}{|c|}{$\frac{\text { Recovery }}{(\%)}$}} \\
\hline & & & & & & & & & & & & \\
\hline & & & 20 & 100 & 20 & 100 & & & 10 & 100 & 10 & 100 \\
\hline & & & \multicolumn{4}{|c|}{$(\mu \mathrm{g} / \mathrm{kg})$} & & & \multicolumn{4}{|c|}{$(\mu \mathrm{g} / \mathrm{kg})$} \\
\hline Furathiocarb & 4.71 & 9 & 17.65 & 8.65 & 99.6 & 82.96 & 3.19 & 10.68 & 12.36 & 17.85 & 89.26 & 90.53 \\
\hline Giberellic acid & 3.02 & 10.05 & 14.36 & 7.45 & 93.6 & 94.33 & 4.39 & 11.33 & 10.54 & 19.63 & 88.09 & 91.53 \\
\hline Halfenprox & 2.46 & 21 & 17.89 & 14.12 & 86.83 & 95.57 & 8.08 & 5.83 & 11.57 & 15.36 & 90.08 & 100.83 \\
\hline $\begin{array}{l}\text { Haloxyfop-2- } \\
\text { Etoxyethyl }\end{array}$ & 4.83 & 16.09 & 17.25 & 15.36 & 96.2 & 86.59 & 4.78 & 10.8 & 12.65 & 9.36 & 85.11 & 91.07 \\
\hline Haloxyfop-P-Methyl & 5.13 & 17.09 & 14.56 & 14.36 & 92.24 & 87.72 & 6.37 & 11.98 & 13.25 & 12.36 & 93.47 & 90.46 \\
\hline Hexaconazole & 5.54 & 18.45 & 14.85 & 21.36 & 96.87 & 89.58 & 5.76 & 1.84 & 14.32 & 10.15 & 92.45 & 82.71 \\
\hline Hexaflumuron & 3.76 & 12.54 & 12.65 & 11.32 & 97.06 & 87.05 & 8.27 & 1.7 & 14.58 & 15.36 & 91.03 & 90.08 \\
\hline Hexythiazox & 4.32 & 14.41 & 15.23 & 11.25 & 93.21 & 91.54 & 5.66 & 2.64 & 10.32 & 14.36 & 86.62 & 90.99 \\
\hline Imazalil & 6.01 & 20.03 & 12.14 & 14.32 & 94.41 & 87.12 & 7.31 & 11.99 & 10.74 & 11.36 & 92.99 & 91.16 \\
\hline Imazamox & 4.44 & 14.81 & 18.23 & 12.36 & 88.87 & 86.71 & 6.8 & 15.87 & 11.35 & 11.32 & 89.33 & 87.18 \\
\hline Imazapic & 4.88 & 16.27 & 15.65 & 11.17 & 89.11 & 89.99 & 10.15 & 13.31 & 12.65 & 11.25 & 78.75 & 88.97 \\
\hline Imazapyr & 3.07 & 10.24 & 18.56 & 10.12 & 86.91 & 86.63 & 11.8 & 10.65 & 16.74 & 29.11 & 85.39 & 99.31 \\
\hline Imazethapyr & 4.75 & 15.84 & 14.89 & 11.14 & 87.7 & 85.85 & 7.56 & 14.63 & 17.96 & 12.36 & 89.51 & 90.05 \\
\hline Imidacloprid & 4.12 & 13.73 & 9.63 & 18.69 & 82.97 & 92.52 & 7.38 & 26.94 & 11.85 & 11.17 & 90.84 & 91.09 \\
\hline Iodosulfuron-Methyl & 2.87 & 9.57 & 6.52 & 12.32 & 86.94 & 85.14 & 7.49 & 15.94 & 14.96 & 10.12 & 85.23 & 86.93 \\
\hline Ioxynil & 2.42 & 8.08 & 5.36 & 11.41 & 93.64 & 83.96 & 6.88 & 21.25 & 18.74 & 11.14 & 90.35 & 86.71 \\
\hline Iprodione & 0.49 & 1.63 & 6.89 & 17.24 & 85.09 & 86.97 & 7.38 & 19.2 & 15.65 & 13.36 & 90.9 & 87.3 \\
\hline Isoxaflutole & 3.01 & 10.02 & 8.47 & 9.63 & 101.63 & 94.06 & 7.2 & 27.58 & 16.32 & 14.63 & 89.68 & 91.93 \\
\hline Kresoxim methyl & 5.85 & 19.5 & 8.45 & 8 & 92.88 & 88.11 & 3.96 & 18.87 & 15.32 & 15.32 & 85.02 & 86.56 \\
\hline Lambda Cyhalothrin & 3.26 & 10.86 & 1.23 & 1.8 & 93.48 & 85.43 & 5.94 & 24.38 & 14.85 & 17.45 & 91.03 & 95.71 \\
\hline Lenacil & 2.4 & 8.12 & 9.62 & 8.24 & 94.7 & 90.01 & 7.2 & 22.67 & 17.85 & 14.11 & 94.58 & 91.53 \\
\hline Lufenuron & 4.97 & 16.56 & 7.21 & 7.65 & 92.49 & 90.03 & 4.45 & 33.83 & 9.32 & 9.45 & 92.08 & 92.44 \\
\hline Malathion & 4.14 & 13.8 & 8.24 & 14.85 & 90.09 & 93.99 & 4.24 & 39.35 & 6.21 & 14.65 & 83.14 & 93.37 \\
\hline MCPA & 4.42 & 14.75 & 9.65 & 17.85 & 93.67 & 85.93 & 5.78 & 25.2 & 8.32 & 17.23 & 98.84 & 88.68 \\
\hline Mecoprop & 3.51 & 11.69 & 8.21 & 9.32 & 96.84 & 87.24 & 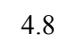 & 24.6 & 8.14 & 15.21 & 91.02 & 96.15 \\
\hline Mefenpyr-diethyl & 5.55 & 18.51 & 9.32 & 6.2 & 90.2 & 82.44 & 3.49 & 24.96 & 9.12 & 14.32 & 85.58 & 90.69 \\
\hline Mesosulfuron methyl & 5.94 & 19.81 & 10.65 & 8.32 & 83.83 & 84.01 & 5.82 & 22.93 & 8.14 & 12.63 & 77.87 & 91.93 \\
\hline Mesotrione & 3.3 & 11.01 & 11.45 & 8.14 & 91.1 & 86.82 & 4.84 & 24.58 & 5.12 & 14.36 & 82.08 & 89.28 \\
\hline Metalaxyl & 4.69 & 15.65 & 18.63 & 9.12 & 90.53 & 91.51 & 8.6 & 24.02 & 4.12 & 12.31 & 82.96 & 96.41 \\
\hline Metamitron & 3.6 & 12 & 14.85 & 14.54 & 91.53 & 94.7 & 6.69 & 13.19 & 6.18 & 11.32 & 94.33 & 98.44 \\
\hline Metconazole & 4.66 & 15.54 & 15.65 & 13.65 & 100.83 & 91.65 & 4.07 & 19.79 & 6.45 & 9.74 & 95.57 & 92.27 \\
\hline Methidathion & 5.16 & 17.19 & 12.32 & 14.52 & 91.07 & 87.26 & 4.32 & 23.99 & 8.12 & 4.65 & 86.59 & 92.02 \\
\hline Methomyl & 4.08 & 13.59 & 14.15 & 18.65 & 90.46 & 85.96 & 4.2 & 14.85 & 9.15 & 6.18 & 87.72 & 89.77 \\
\hline Methoxyfenozide & 4.13 & 13.77 & 14.78 & 14.65 & 82.71 & 89.59 & 4.75 & 14.13 & 9.12 & 6.45 & 89.58 & 96.25 \\
\hline Metolachlor & 5.98 & 19.93 & 14.56 & 17.96 & 90.08 & 94.47 & 5.04 & 19.26 & 10.12 & 8.12 & 87.05 & 93.4 \\
\hline Metrubuzin & 1.34 & 4.47 & 18.45 & 11.85 & 90.99 & 86.83 & 3.23 & 16.01 & 15.14 & 9.15 & 91.54 & 90.22 \\
\hline Metsulfuron-methyl & 3.32 & 11.07 & 14.52 & 14.96 & 91.16 & 91.52 & 2.43 & 11.64 & 14.12 & 9.12 & 87.12 & 96.98 \\
\hline Monocrotophos & 1.27 & 4.23 & 12.54 & 18.74 & 87.18 & 87.68 & 5.01 & 19.4 & 11.1 & 10.12 & 86.71 & 96.66 \\
\hline Monolinuron & 1.64 & 5.46 & 16.52 & 15.65 & 88.97 & 83.56 & 6.17 & 16.12 & 13.1 & 15.14 & 89.99 & 99.51 \\
\hline Myclobutanil & 4.34 & 14.48 & 18.25 & 16.32 & 99.31 & 93.36 & 0.13 & 28.67 & 17.23 & 14.12 & 86.63 & 93.42 \\
\hline Nicosulfuron & 4.99 & 16.63 & 14.65 & 15.32 & 90.05 & 84.52 & 3.09 & 22.31 & 15.21 & 11.1 & 85.85 & 97.25 \\
\hline
\end{tabular}




$$
-6899-
$$

\begin{tabular}{|c|c|c|c|c|c|c|c|c|c|c|c|c|}
\hline \multirow{5}{*}{ Pesticides } & \multicolumn{6}{|c|}{ Cucumber } & \multicolumn{6}{|c|}{ Grapefruit } \\
\hline & \multirow{4}{*}{ LOD } & \multirow{4}{*}{ LOQ } & \multirow{2}{*}{\multicolumn{2}{|c|}{$\begin{array}{l}\text { Precision } \\
\text { RSD (\%) }\end{array}$}} & \multirow{2}{*}{\multicolumn{2}{|c|}{$\frac{\text { Recovery }}{(\%)}$}} & \multirow{4}{*}{ LOD } & \multirow{4}{*}{ LOQ } & \multirow{2}{*}{\multicolumn{2}{|c|}{$\frac{\text { Precision }}{\text { RSD (\%) }}$}} & \multirow{2}{*}{\multicolumn{2}{|c|}{$\frac{\text { Recovery }}{(\%)}$}} \\
\hline & & & & & & & & & & & & \\
\hline & & & 20 & 100 & 20 & 100 & & & 10 & 100 & 10 & 100 \\
\hline & & & \multicolumn{4}{|c|}{$(\mu \mathrm{g} / \mathrm{kg})$} & & & \multicolumn{4}{|c|}{$(\mu \mathrm{g} / \mathrm{kg})$} \\
\hline Novaluron & 3.29 & 10.95 & 14.32 & 14.85 & 91.09 & 88.19 & 3.39 & 13.56 & 14.32 & 13.1 & 92.52 & 96.79 \\
\hline Omethoate & 1.48 & 4.93 & 13.21 & 17.85 & 86.93 & 87.11 & 2.09 & 14.41 & 12.63 & 10.21 & 85.14 & 90.71 \\
\hline Oxadixyl & 3.46 & 11.53 & 15.36 & 9.32 & 86.71 & 87.44 & 4.23 & 14.01 & 14.36 & 10.14 & 83.96 & 98.12 \\
\hline Oxamyl & 1.27 & 4.23 & 14.36 & 6.21 & 87.3 & 86.5 & 2.68 & 15.82 & 12.31 & 9.85 & 86.97 & 91.6 \\
\hline Oxyflourfen & 4.77 & 15.91 & 11.36 & 8.32 & 91.93 & 83.48 & 4.18 & 16.79 & 11.32 & 15.17 & 94.06 & 99.08 \\
\hline Parathion Methyl & 1.28 & 4.28 & 11.32 & 8.14 & 86.56 & 93.11 & 5.81 & 10.75 & 9.65 & 11.12 & 88.11 & 86.35 \\
\hline Penconazole & 5.93 & 19.77 & 11.25 & 9.12 & 95.71 & 83.77 & 3.56 & 8.1 & 10.32 & 12.23 & 85.43 & 96.45 \\
\hline Pendimethalin & 4.48 & 14.95 & 24.32 & 8.14 & 91.53 & 90.2 & 1.8 & 16.68 & 11.85 & 18.32 & 90.01 & 92.42 \\
\hline Permethrin & 4.99 & 16.62 & 12.36 & 14.12 & 92.44 & 92.26 & 1.24 & 20.58 & 14.65 & 14.36 & 90.03 & 90.57 \\
\hline Phenmedipham & 4.38 & 14.59 & 11.17 & 11.12 & 93.37 & 92.3 & 2.33 & 0.42 & 17.64 & 9.15 & 93.99 & 90.06 \\
\hline Phenthoate & 5.99 & 19.97 & 10.12 & 10.12 & 88.68 & 95.18 & 3.65 & 10.3 & 8.65 & 9.12 & 85.93 & 85.16 \\
\hline Phosalone & 5.89 & 19.64 & 11.14 & 18.26 & 96.15 & 85.34 & 2.43 & 11.3 & 7.65 & 10.32 & 87.24 & 93.31 \\
\hline Phosmet & 6.36 & 21.21 & 15.36 & 17.32 & 90.69 & 84.19 & 4.49 & 6.96 & 8.32 & 11.25 & 82.44 & 93.71 \\
\hline Pirimicarb & 2.38 & 7.92 & 18.36 & 14.32 & 91.93 & 93.02 & 4.58 & 14.1 & 9.63 & 10.87 & 84.01 & 86.76 \\
\hline Pirimiphos methyl & 4.55 & 15.17 & 12.35 & 18.65 & 89.28 & 88.01 & 2.83 & 8.92 & 12.54 & 9.63 & 86.82 & 89.6 \\
\hline Prochloraz & 4.03 & 13.43 & 14.36 & 12.74 & 96.41 & 90.23 & 4.01 & 13.92 & 14.56 & 8.35 & 91.51 & 102.06 \\
\hline Procymidone & 4.22 & 14.08 & 17.96 & 9.12 & 98.44 & 87.9 & 0.65 & 19.37 & 17.85 & 8.65 & 94.7 & 95.94 \\
\hline Profenofos & 6.06 & 20.19 & 11.85 & 10.11 & 92.27 & 91.11 & 4.95 & 11.87 & 19.63 & 7.65 & 91.65 & 100.07 \\
\hline $\begin{array}{l}\text { Profoxydim } \\
\text { (Clefoxydim) }\end{array}$ & 2.13 & 7.12 & 14.96 & 17.85 & 92.02 & 88.23 & 5.11 & 5.99 & 15.36 & 8.32 & 87.26 & 86.06 \\
\hline Prometryn & 4.97 & 16.57 & 18.74 & 19.63 & 89.77 & 93.85 & 5.82 & 4.12 & 19.36 & 9.63 & 85.96 & 94.31 \\
\hline Propaquizafop & 4.81 & 16.05 & 15.65 & 15.36 & 96.25 & 87.19 & 4.9 & 7.76 & 17.36 & 12.54 & 89.59 & 92.67 \\
\hline Propargite & 5.55 & 18.48 & 16.32 & 19.36 & 93.4 & 96.29 & 2.76 & 12.16 & 16.15 & 14.56 & 94.47 & 100.31 \\
\hline Propazine & 4.28 & 14.26 & 15.32 & 21.36 & 90.22 & 84.29 & 4.97 & 8.11 & 15.17 & 17.85 & 86.83 & 92.13 \\
\hline Propoxycarbazone & 3.96 & 13.21 & 14.85 & 10.12 & 96.98 & 87.32 & 2.74 & 14.97 & 11.12 & 39.47 & 91.52 & 96.42 \\
\hline Propyconazole & 5.7 & 19.01 & 17.85 & 14.25 & 96.66 & 84.74 & 4.61 & 15.28 & 12.23 & 17.96 & 87.68 & 87.28 \\
\hline Propyzamide & 4.37 & 14.56 & 21.36 & 9.15 & 99.51 & 89.42 & 3.73 & 9.44 & 18.32 & 11.85 & 83.56 & 86.31 \\
\hline Prothopos & 5.82 & 19.41 & 6.21 & 9.12 & 93.42 & 89.52 & 3.01 & 13.37 & 14.36 & 14.96 & 93.36 & 99.09 \\
\hline Pymetrozine & 0.17 & 0.56 & 8.32 & 10.12 & 97.25 & 85.74 & 0.52 & 2.17 & 9.15 & 18.74 & 84.52 & 92.48 \\
\hline Pyraclostrobin & 5.17 & 17.23 & 8.14 & 15.14 & 96.79 & 83.32 & 2.17 & 16.51 & 9.12 & 15.65 & 88.19 & 85.9 \\
\hline Pyraflufen-Ethyl & 4.21 & 14.02 & 9.12 & 14.12 & 90.71 & 92.91 & 5.36 & 17.02 & 6.45 & 16.32 & 87.11 & 82.99 \\
\hline Pyrazophos & 6.02 & 20.06 & 8.14 & 11.1 & 98.12 & 88.05 & 4.67 & 19.41 & 8.12 & 15.32 & 87.44 & 98.77 \\
\hline Pyridaben & 5.82 & 19.4 & 10.12 & 13.1 & 91.6 & 90.89 & 1.09 & 16.33 & 9.15 & 14.85 & 86.5 & 98.8 \\
\hline Pyridaphention & 5.75 & 19.16 & 10.11 & 10.21 & 99.08 & 96.07 & 3.95 & 9.2 & 9.12 & 17.85 & 83.48 & 97.12 \\
\hline Pyridate & 5.22 & 17.39 & 14.32 & 10.14 & 86.35 & 91.46 & 3.4 & 16.57 & 10.12 & 9.32 & 93.11 & 94.68 \\
\hline Pyriproxyfen & 4.57 & 15.24 & 10.34 & 9.85 & 96.45 & 86.32 & 3.46 & 9.12 & 15.14 & 6.21 & 83.77 & 85 \\
\hline Quizalofop-P-Ethyl & 4.99 & 16.63 & 15.32 & 9.85 & 92.42 & 89.45 & 3.51 & 15.36 & 14.12 & 8.32 & 90.2 & 96.72 \\
\hline Rimsulfuron & 0.55 & 1.82 & 9.98 & 10.25 & 90.57 & 89.54 & 2.08 & 12.44 & 11.1 & 8.14 & 92.26 & 90.67 \\
\hline Sethoxydim & 1.25 & 4.18 & 8.32 & 11.14 & 90.06 & 91.31 & 3.21 & 10.03 & 13.1 & 9.12 & 92.3 & 90.39 \\
\hline Simozine & 2.33 & 7.75 & 7.23 & 14.25 & 85.16 & 90.57 & 2.7 & 1.72 & 10.21 & 8.14 & 95.18 & 95.14 \\
\hline Spirodiclofen & 4.82 & 16.08 & 8.23 & 18.21 & 93.31 & 92.29 & 3.67 & 7.22 & 10.14 & 10.21 & 85.34 & 95.23 \\
\hline Spiroxamine & 2.64 & 8.79 & 6.12 & 14.32 & 93.71 & 99.81 & 4.05 & 17.85 & 9.85 & 10.14 & 84.19 & 94.61 \\
\hline
\end{tabular}




$$
-6900-
$$

\begin{tabular}{|c|c|c|c|c|c|c|c|c|c|c|c|c|}
\hline \multirow{5}{*}{ Pesticides } & \multicolumn{6}{|c|}{ Cucumber } & \multicolumn{6}{|c|}{ Grapefruit } \\
\hline & \multirow{4}{*}{ LOD } & \multirow{4}{*}{ LOQ } & \multirow{2}{*}{\multicolumn{2}{|c|}{$\frac{\text { Precision }}{\text { RSD (\%) }}$}} & \multirow{2}{*}{\multicolumn{2}{|c|}{$\frac{\text { Recovery }}{(\%)}$}} & \multirow{4}{*}{ LOD } & \multirow{4}{*}{ LOQ } & \multirow{2}{*}{\multicolumn{2}{|c|}{$\frac{\text { Precision }}{\text { RSD (\%) }}$}} & \multirow{2}{*}{\multicolumn{2}{|c|}{$\frac{\text { Recovery }}{(\%)}$}} \\
\hline & & & & & & & & & & & & \\
\hline & & & 20 & 100 & 20 & 100 & & & 10 & 100 & 10 & 100 \\
\hline & & & \multicolumn{4}{|c|}{$(\mu \mathrm{g} / \mathrm{kg})$} & & & \multicolumn{4}{|c|}{$(\mu \mathrm{g} / \mathrm{kg})$} \\
\hline Sulfosulfuron & 0.66 & 2.21 & 14.23 & 14.32 & 86.76 & 101.91 & 3.53 & 15.57 & 8.45 & 9.85 & 93.02 & 101.91 \\
\hline Tau-Fluvalinate & 3.29 & 10.96 & 15.21 & 19.32 & 89.6 & 94.87 & 4.68 & 3.62 & 10.11 & 10.12 & 88.01 & 100.96 \\
\hline Tebuconazole & 5.79 & 19.29 & 14.23 & 10.12 & 102.06 & 95.67 & 3.02 & 13.16 & 14.65 & 14.12 & 90.23 & 96.5 \\
\hline Tebufenozide & 5.25 & 17.51 & 9.14 & 15.14 & 95.94 & 104.15 & 4.6 & 11.32 & 17.65 & 15.17 & 87.9 & 104.15 \\
\hline Teflubenzuron & 3.91 & 13.02 & 8.14 & 14.12 & 100.07 & 100.6 & 4.83 & 11.53 & 12.11 & 11.52 & 91.11 & 100.6 \\
\hline Tepraloxydim & 1.69 & 5.62 & 7.56 & 11.1 & 86.06 & 103.01 & 3.06 & 11.71 & 17.15 & 10.54 & 88.23 & 103.01 \\
\hline Terbuthylazine & 3.55 & 11.82 & 8.14 & 13.1 & 94.31 & 102.61 & 5.62 & 6.94 & 19.12 & 11.57 & 93.85 & 102.61 \\
\hline Terbutryn & 5.82 & 19.41 & 8.65 & 10.21 & 92.67 & 95.62 & 3.88 & 10.71 & 13.25 & 12.65 & 87.19 & 93.95 \\
\hline Tetraconazole & 4.73 & 15.77 & 7.12 & 10.14 & 100.31 & 93.73 & 3.19 & 8.99 & 10.11 & 13.25 & 96.29 & 99.51 \\
\hline Thiabendazole & 2.56 & 8.53 & 8.45 & 9.85 & 92.13 & 94.54 & 3.49 & 12.23 & 9.85 & 14.32 & 84.29 & 98.75 \\
\hline Thiacloprid & 1.57 & 5.22 & 8.69 & 10.12 & 96.42 & 99.77 & 6.3 & 13.49 & 8.63 & 14.58 & 87.32 & 99.77 \\
\hline Thiamethoaxam & 0.70 & 2.35 & 6.25 & 14.12 & 87.28 & 101.08 & 4.33 & 11.78 & 7.98 & 12.69 & 84.74 & 101.08 \\
\hline $\begin{array}{c}\text { Thifensulfuron- } \\
\text { methyl }\end{array}$ & 0.27 & 0.91 & 5.12 & 15.17 & 86.31 & 102.61 & 2.96 & 15.6 & 7.63 & 11.36 & 89.42 & 102.61 \\
\hline Thiohanate methyl & 2.73 & 9.09 & 4.12 & 11.12 & 99.09 & 102.27 & 3.7 & 10.05 & 7.52 & 10.87 & 89.52 & 102.27 \\
\hline Thiometon & 4.12 & 13.72 & 6.18 & 12.23 & 92.48 & 102.82 & 1.4 & 15.35 & 9.41 & 9.15 & 85.74 & 102.82 \\
\hline Tolyfluanid & 3.21 & 10.68 & 6.45 & 18.32 & 85.9 & 92.82 & 4.21 & 16.11 & 7.45 & 8.32 & 83.32 & 97.51 \\
\hline Tralkoxydim & 3.4 & 11.33 & 8.12 & 14.36 & 82.99 & 98.99 & 4.26 & 10.2 & 11.25 & 9.74 & 92.91 & 98.26 \\
\hline Triadimefon & 1.75 & 5.83 & 9.15 & 9.15 & 98.77 & 99.6 & 5.78 & 18.73 & 10.87 & 6.32 & 88.05 & 99.6 \\
\hline Triadimenol & 3.24 & 10.8 & 9.12 & 9.12 & 98.8 & 101.72 & 5.18 & 12.93 & 9.63 & 7.14 & 90.89 & 101.72 \\
\hline Triallate & 3.59 & 11.98 & 10.12 & 10.12 & 97.12 & 101.45 & 5.14 & 10.63 & 8.35 & 6.32 & 96.07 & 101.45 \\
\hline Triasulfuron & 0.55 & 1.84 & 15.14 & 15.14 & 94.68 & 98.88 & 3.27 & 11.64 & 8.65 & 7.52 & 91.46 & 98.88 \\
\hline Tribenuron-Methyl & 0.51 & 1.7 & 14.12 & 14.12 & 85 & 101.79 & 1.79 & 20.99 & 7.65 & 10.21 & 86.32 & 101.79 \\
\hline Trichlorfon & 0.79 & 2.64 & 11.1 & 11.1 & 96.72 & 99.8 & 3.2 & 14.42 & 8.32 & 10.14 & 89.45 & 99.8 \\
\hline Trifloxystrobin & 3.6 & 11.99 & 13.1 & 13.1 & 90.67 & 102.21 & 3.04 & 9.86 & 9.63 & 9.85 & 89.54 & 102.21 \\
\hline Triflumizole & 4.76 & 15.87 & 10.21 & 10.21 & 90.39 & 100.93 & 2.56 & 12.33 & 12.54 & 10.12 & 91.31 & 100.93 \\
\hline Triflumuron & 3.99 & 13.31 & 10.14 & 10.14 & 95.14 & 102.11 & 1.51 & 4.67 & 14.56 & 14.12 & 90.57 & 102.11 \\
\hline Triticonazole & 3.19 & 10.65 & 9.85 & 9.85 & 95.23 & 101.54 & 4.63 & 14.03 & 17.85 & 15.17 & 92.29 & 101.54 \\
\hline $2.4 \mathrm{D}$ Acid & 4.39 & 14.63 & 7.65 & 11.23 & 94.61 & 102.37 & 2.07 & 14.21 & 14.12 & 9.12 & 99.81 & 102.37 \\
\hline
\end{tabular}

LOD: limit of detection $(\mu \mathrm{g} / \mathrm{kg})$; LOQ: limit of quantification $(\mu \mathrm{g} / \mathrm{kg})$

Calculated at $20 \mu \mathrm{g} / \mathrm{kg}$ and $100 \mu \mathrm{g} / \mathrm{kg}$ in fortificated cucumber matrix

Calculated at $10 \mu \mathrm{g} / \mathrm{kg}$ and $100 \mu \mathrm{g} / \mathrm{kg}$ in fortificated grapefruit matrix

The fungicides metalxyl and procymidone were found in 4 cucumber samples at levels varying from 0.011 to $0.039 \mathrm{mg} \mathrm{kg}^{-1}$ and from 0.014 to $0.033 \mathrm{mg} \mathrm{kg}^{-1}$, respectively. The insecticide chlorpyrifos was detectable only in 1 cucumber samples, with a mean concentration of $0.037 \mathrm{mg} \mathrm{kg}^{-1}$. Similarly, two cucumber samples were found to contain iprodione, Carbendazim, which is an authorised neonicotinoid insecticide. The concentrations of iprodione, carbendazim in positive samples were from 0.022 to $0.029 \mathrm{mg} \mathrm{kg}^{-1}$ and from 0.014 to $0.032 \mathrm{mg} \mathrm{kg}^{-1}$, respectively.

The results show that grapefruits had slightly higher frequencies $(13.8 \%)$ of pesticide residues than cucumber samples $(11.6 \%)$, but the number of residues was less. Of the 
233 target analytes monitored, only 9 pesticide residues were found in grapefruits at concentrations varying from 0.002 to $0.042 \mathrm{mg} \mathrm{kg}^{-1}$; none above the MRL. Multiple residues in grapefruits were found in $3.5 \%$ of the samples. While 15 grapefruit samples contained two residues of pesticides, only 2 samples were contaminated with three pesticide residues.

The fungicide fludioxonil was the most prominent pesticide found in grapefruit samples, with a frequency of $9.1 \%$, and with levels ranging from 0.019 to $0.041 \mathrm{mg} \mathrm{kg}^{-1}$. Fludioxonil, which belongs to the class of phenylpyrrole, is used to control a broad spectrum of plant diseases in various vegetables. The acute toxicity of fludioxnil is low. Fludioxonil did not have carcinogenic potential (FAO, 2012).

Imazalil was the second most commonly found pesticide in grapefruits with $4.7 \%$ of the samples. The results obtained from the second and third year showed that 7 samples contained imazalil at levels varying from 0.017 to $0.102 \mathrm{mg} \mathrm{kg}^{-1}$, and from 0.021 to $0.109 \mathrm{mg} \mathrm{kg}{ }^{-1}$, respectively. In the first year, 4 samples had this residue at concentrations ranging from 0.016 to $0.032 \mathrm{mg} \mathrm{kg}^{-1}$.

Thiabendazol was detected in 7 samples at levels ranging from 0.014 to $0.081 \mathrm{mg} \mathrm{kg}^{-1}$, of which 5 samples contained simultaneously the fungicide quinophos. Thiabendazol, belongs to the class of benzimidazole. It is a systemic fungicide used in citrus fruits such as orange, lemon and grapefruit against Penicillium digitatum.

The insecticide carbaryl was present in 4 grapefruit samples, with a mean concentration of $0.028 \mathrm{mg} \mathrm{kg}^{-1}$. Carbaryl belongs to the class of carbamate. It is a pesticide and plant growth regulator used in citrus fruits such as orange, lemon, grapefruit.

\section{Uncertainty assessment}

The results of method uncertainty assessment considering six sources are presented in Table 2. For cucumber samples the overall contributions to $U_{c}$ of the method of the uncertainties associated with $\mathrm{U}_{1}$ (standard solution preparation), $\mathrm{U}_{2}$ (calibration curve preparation), $\mathrm{U}_{3}$ (sample preparation treatment), $\mathrm{U}_{4}$ (precision), and $\mathrm{U}_{5}$ (accuracy/bias) ranged from $5.3 \%$ to $10.6 \%$ and $\mathrm{U}_{6}$ (linear least square fitting) ranged from $85.6 \%$ to $93.1 \%$. In the same way, for grapefruit, the overall contributions of the uncertainties associated with $\mathrm{U}_{1}, \mathrm{U}_{2}, \mathrm{U}_{3}, \mathrm{U}_{4}$, and $\mathrm{U}_{5}$ varied from $5.1 \%$ to $11.8 \%$ and $\mathrm{U}_{6}$ ranged from $87.8 \%$ to $95.1 \%$. In the light of these results, the uncertainty associated with u6 presents the highest contribution for all the pesticides under study in cucember and grapefruit.

As can be seen in Table 2, $\mathrm{U}_{\mathrm{e}}$ values at $95 \%$ confidence level for cucember ranged from $12.3 \%$ to $18.7 \%$ and for grapefruit from $15.6 \%$ to $23.6 \%$. $\mathrm{U}_{\mathrm{e}}$ values of all pesticides detected in cucumber samples generally offer lower values than grapefruit. For all target analytes, the $U_{\mathrm{e}}$ was within the criteria specified in the SANTE Guideline (less than 50\%). These results are similar to those reported for methods using GC-MS, LCMS-MS and the QuEChERS procedure. For example, Walorczyk (2014) reported $U_{e}$ values ranging from $7.0 \%$ to $53.0 \%$ for samples of black currant, where the majority of pesticides had a uncertainty of $<30.0 \%$. In addition, da Silva Sousa et al. (2018) reported $U_{\mathrm{e}}$ values from $7.9 \%$ to $36.1 \%$ for melon samples. At first glance, the results of this method are high, but the uncertainty acceptance criteria according to the European SANTE / 11945/2015 guideline European Commission (2015) is the time when the value is $50.0 \%$. Although it is recommended to use the internal standard to minimize uncertainties, the proposed method indicated that expanded uncertainties are not necessary. 
Table 2. Method uncertainties estimated for different sources and, combined $\left(U_{c}\right)$ and expanded $\left(U_{e}\right)$ uncertainty

\begin{tabular}{|c|c|c|c|c|c|c|c|c|c|c|c|c|c|c|c|c|}
\hline \multirow{3}{*}{ Pesticide } & \multicolumn{8}{|c|}{ Cucumber } & \multicolumn{8}{|c|}{ Grapefruit } \\
\hline & \multirow[b]{2}{*}{$\mathbf{U}_{1}$} & \multirow[b]{2}{*}{$\mathbf{U}_{2}$} & \multirow{2}{*}{$\begin{array}{c}\begin{array}{c}\mathrm{U}_{\mathrm{i}}^{\mathrm{a}} \\
(\mathrm{mg} / \mathrm{kg} \\
(\%)\end{array} \\
\mathrm{U}_{3}\end{array}$} & \multirow[b]{2}{*}{$\begin{array}{r}\mathbf{U}_{4} \\
(\mathrm{mg} / \mathrm{kg})\end{array}$} & \multirow[b]{2}{*}{$\mathbf{U}_{6}$} & \multirow[b]{2}{*}{$\mathbf{U}_{\mathrm{c}}$} & \multirow[b]{2}{*}{$\begin{array}{c}\mathbf{U}_{\mathbf{e}}^{\mathbf{b}} \\
(\mathrm{mg} / \mathrm{kg} \\
\quad) \\
\end{array}$} & \multirow[b]{2}{*}{$\begin{array}{c}\mathbf{U}_{\mathbf{e}}(\% \\
)\end{array}$} & \multirow[b]{2}{*}{$\mathbf{U}_{1}$} & \multirow[b]{2}{*}{$\mathbf{U}_{2}$} & \multirow{2}{*}{$\begin{array}{c}\begin{array}{c}\mathrm{U}_{\mathrm{i}}^{\mathrm{a}} \\
(\mathrm{mg} / \mathrm{kg} \\
(\%))\end{array} \\
\mathrm{U}_{3} \\
\end{array}$} & \multirow[b]{2}{*}{$\begin{array}{r}\mathbf{U}_{4} \\
(\mathrm{mg} / \mathrm{kg})\end{array}$} & \multirow[b]{2}{*}{$\mathbf{U}_{6}$} & \multirow[b]{2}{*}{$\mathbf{U}_{\mathbf{c}}$} & \multirow[b]{2}{*}{$\begin{array}{c}\mathbf{U}_{\mathbf{e}}^{\mathbf{b}} \\
(\mathrm{mg} / \mathrm{kg} \\
)\end{array}$} & \multirow[b]{2}{*}{$\begin{array}{l}\mathbf{U}_{\mathbf{e}}(\%) \\
)\end{array}$} \\
\hline & & & & & & & & & & & & & & & & \\
\hline Trifluralin & $0.044(2.3)$ & $0.036(1.0)$ & $0.031(0.9)$ & $0.023(0.7) 0.039$ & 0.301 & 0.313 & 0.618 & 15.6 & $0.044(2.3)$ & $0.036(1.0)$ & $0.031(0.9)$ & $0.050(1.7) 0.064(2.8)$ & $0.350(88.4)$ & 0.384 & 0.765 & 15.7 \\
\hline Chlorothalonil & $0.044(2.1)$ & $0.036(0.9)$ & $0.031(0.8)$ & $0.072(4.3) 0.032$ & 0.317 & 0.311 & 0.662 & 15.8 & $0.044(2.1)$ & $0.036(0.9)$ & $0.031(0.8)$ & $0.034(1.2) 0.044(1.7)$ & $0.318(87.8)$ & 0.382 & 0.665 & 16.4 \\
\hline Formothion & $0.044(2.0)$ & $0.036(1.2)$ & $0.031(1.5)$ & 0.008 & 0.245 & 0.320 & 0.665 & 16.2 & $0.044(2.0)$ & $0.036(1.2)$ & $0.031(1.5)$ & $0.076(2.2) 0.068(1.6)$ & $0.333(92.1)$ & 0.381 & 0.654 & 18.3 \\
\hline Vinclozolin & $0.044(3.2)$ & $0.036(1.1)$ & 0.031 & 0.007 & 0.298 & 0.254 & 0.514 & 16.3 & $0.044(3.2)$ & $0.036(1.1)$ & 0.031 & $0.071(4.4) 0.060(3.5)$ & $0.368(91.2)$ & 0.504 & 0.774 & 19.5 \\
\hline Fenitrothion & $0.044(3.5)$ & $0.036(1.5)$ & $0.031(1.0)$ & 0.009 & 0.332 & 0.256 & 0.512 & 15.6 & $0.044(3.5)$ & $0.036(1.5)$ & $0.031(1.0)$ & $0.097(6.3) 0.071(3.4)$ & $0.495(95.1)$ & 0.501 & 0.771 & 15.6 \\
\hline Dicofol & $0.044(1.9)$ & $0.036(1.6)$ & 0.031 & 0.070 & $0.312(92.4)$ & 0.245 & 0.628 & 12.6 & $0.044(1.9)$ & $0.036(1.6)$ & 0.031 & $0.096(5.4) 0.062(2.6)$ & $0.452(94.1)$ & 0.359 & 0.659 & 16.2 \\
\hline Captan & $0.044(2.0)$ & $0.036(1.7)$ & 0.031 & $0.066(0.2) 0.041$ & 0.298 & 0.287 & 0.624 & 12.8 & $0.044(2.0)$ & $0.036(1.7)$ & 0.031 & $0.071(2.1) 0.045(1.6)$ & $0.413(92.1)$ & 0.385 & 0.662 & 19.6 \\
\hline Folpet & $0.044(3.4)$ & $0.036(1.2)$ & 0.031 & $0.052(0.1) 0.068$ & 0.291 & 0.298 & 0.701 & 12.9 & $0.044(3.4)$ & $0.036(1.2)$ & 0.031 & $0.033(1.5) 0.061(3.2)$ & $0.402(90.8)$ & 0.504 & 0.652 & 20.3 \\
\hline Qinomethionate & $0.044(3.3)$ & $0.036(1.4)$ & $0.031(0.9)$ & $0.054(0.6) 0.071$ & 0.335 & 0.301 & 0.704 & 12.7 & $0.044(3.3)$ & $0.036(1.4)$ & $0.031(0.9)$ & $0.051(1.9) 0.070(3.3)$ & 0.350 & 0.501 & 1.014 & 21.6 \\
\hline Dinobuton & $0.044(3.2)$ & $0.036(1.5)$ & 0.031 & $0.051(0.7) 0.065$ & $0.301(92.1)$ & 0.305 & 0.517 & 12.8 & $0.044(3.2)$ & $0.036(1.5)$ & 0.031 & $0.076(2.2) 0.064(2.8)$ & $0.318(87.8)$ & 0.328 & 0.665 & 23.4 \\
\hline Endosulfan A & $0.044(3.1)$ & $0.036(1.2)$ & 0.031 & $0.041(0.5) 0.070$ & 0.317 & 0.321 & 0.514 & 13.1 & $0.044(3.1)$ & $0.036(1.2)$ & 0.031 & $0.071(4.4) 0.044(1.7)$ & $0.333(92.1)$ & 0.329 & 1.011 & 21.8 \\
\hline Endosulfan B & $0.044(2.1)$ & $0.036(1.3)$ & 0.031 & 0.040 & 0.245 & 0.315 & 0.548 & 13.6 & $0.044(2.1)$ & $0.036(1.3)$ & 0.031 & $0.097(6.3) 0.068(1.6)$ & $0.368(91.2)$ & 0.385 & 0.774 & 22.8 \\
\hline Bromopropylate & $0.044(3.5)$ & $0.036(1.0)$ & $0.031(0.9)$ & $0.043(0.2) 0.039$ & 0.298 & 0.311 & 0.621 & 13.8 & $0.044(3.5)$ & $0.036(1.0)$ & $0.031(0.9)$ & $0.096(5.4) 0.060(3.5)$ & $0.495(95.1)$ & 0.384 & 0.776 & 21.8 \\
\hline Tetradifon & $0.044(3.3)$ & $0.036(0.9)$ & $0.031(0.7)$ & 0.054 & $0.332(85.6)$ & 0.314 & 0.514 & 13.9 & $0.044(3.3)$ & $0.036(0.9)$ & 0.031 & $0.071(2.1) 0.071(3.4)$ & $0.452(94.1)$ & 0.391 & 0.778 & 23.6 \\
\hline $\begin{array}{c}\text { Alfa- } \\
\text { Cypermethrin a }\end{array}$ & $0.044(2.3)$ & $0.036(1.2)$ & $0.031(0.8)$ & $0.071(0.3) 0.038$ & 0.312 & 0.317 & 0.665 & 14.6 & $0.044(2.3)$ & $0.036(1.2)$ & $0.031(0.8)$ & $0.033(1.5) 0.062(2.6)$ & $0.413(92.1)$ & 0.398 & 0.662 & 20.8 \\
\hline $\begin{array}{c}\text { Alfa- } \\
\text { Cypermethrin b }\end{array}$ & $0.044(2.1)$ & $0.036(1.1)$ & $0.031(0.9)$ & $0.041(0.5) 0.039$ & 0.298 & 0.298 & 0.541 & 15.8 & $0.044(2.1)$ & $0.036(1.1)$ & $0.031(0.9)$ & $0.051(1.9) 0.045(1.6)$ & $0.402(90.8)$ & 0.387 & 1.010 & 19.6 \\
\hline alpha.-BHC & $0.044(2.0)$ & $0.036(1.5)$ & $0.031(1.0)$ & $0.040(0.6) 0.041(2.1)$ & 0.291 & 0.289 & 0.526 & 14.9 & $0.044(2.0)$ & $0.036(1.5)$ & $0.031(1.0)$ & $0.076(2.2) 0.061(3.2)$ & $0.350(88.4)$ & 0.384 & 0.669 & 18.9 \\
\hline delta.-BHC & $0.044(3.2)$ & $0.036(1.6)$ & 0.031 & $0.043(0.2) 0.068$ & 0.331 & 0.287 & 0.668 & 15.6 & $0.044(3.2)$ & $0.036(1.6)$ & 0.031 & $0.071(4.4) 0.070(3.3)$ & 0.318 & 0.385 & 0.771 & 17.6 \\
\hline Heptachlor & $0.044(3.5)$ & $0.036(1.7)$ & 0.031 & $0.054(0.4) 0.032(1.5)$ & 0.301 & 0.269 & 0.662 & 16.9 & $0.044(3.5)$ & $0.036(1.7)$ & 0.031 & $0.097(6.3) 0.064(2.8)$ & $0.333(92.1)$ & 0.381 & 0.778 & 15.9 \\
\hline 2,4-DDE & $0.044(1.9)$ & $0.036(1.4)$ & 0.031 & $0.071(0.3) 0.039$ & 0.317 & 0.278 & 0.669 & 16.8 & $0.044(1.9)$ & $0.036(1.4)$ & 0.031 & $0.096(5.4) 0.044(1.7)$ & $0.368(91.2)$ & 0.329 & 0.668 & 16.3 \\
\hline
\end{tabular}




\begin{tabular}{|c|c|c|c|c|c|c|c|c|c|c|c|c|c|c|c|c|}
\hline \multirow{3}{*}{ Pesticide } & \multicolumn{8}{|c|}{ Cucumber } & \multicolumn{8}{|c|}{ Grapefruit } \\
\hline & \multirow[b]{2}{*}{$\mathbf{U}_{1}$} & \multirow[b]{2}{*}{$\mathbf{U}_{2}$} & \multirow{2}{*}{$\begin{array}{c}\begin{array}{c}\mathrm{U}_{\mathrm{i}}^{\mathrm{a}} \\
(\mathrm{mg} / \mathrm{kg}\end{array} \\
(\%))\end{array}$} & \multirow[b]{2}{*}{$\begin{array}{l}\mathbf{U}_{4} \\
\mathrm{~kg})\end{array}$} & \multirow[b]{2}{*}{$\mathbf{U}_{6}$} & \multirow[b]{2}{*}{$\mathbf{U}_{\mathbf{c}}$} & \multirow[b]{2}{*}{$\begin{array}{c}\mathbf{U}_{\mathbf{e}}^{\mathbf{b}} \\
(\mathrm{mg} / \mathrm{kg} \\
)\end{array}$} & \multirow[b]{2}{*}{$\begin{array}{c}\mathbf{U}_{\mathbf{e}}(\% \\
)\end{array}$} & \multirow[b]{2}{*}{$\mathrm{U}_{1}$} & \multirow[b]{2}{*}{$\mathbf{U}_{2}$} & \multirow{2}{*}{$\begin{array}{c}\begin{array}{c}\mathrm{U}_{\mathrm{i}}^{\mathrm{a}} \\
(\mathrm{mg} / \mathrm{kg} \\
(\%))\end{array} \\
\mathrm{U}_{3}\end{array}$} & \multirow[b]{2}{*}{$\begin{array}{r}\mathbf{U}_{4} \\
(\mathrm{mg} / \mathrm{kg})\end{array}$} & \multirow[b]{2}{*}{$\mathbf{U}_{6}$} & \multirow[b]{2}{*}{$\mathbf{U}_{\mathbf{c}}$} & \multirow[b]{2}{*}{$\begin{array}{c}\mathbf{U}_{\mathbf{e}}^{\mathbf{b}} \\
(\mathrm{mg} / \mathrm{kg} \\
)\end{array}$} & \multirow[b]{2}{*}{$\begin{array}{c}\mathbf{U}_{\mathbf{e}}(\%) \\
)\end{array}$} \\
\hline & & & & & & & & & & & & & & & & \\
\hline Dieldrin & $0.044(2.0)$ & $0.036(1.5)$ & $0.031(0.9)$ & 0.072 & $0.245(90.4)$ & 0.259 & 0.554 & 14.3 & $0.044(2.0)$ & $0.036(1.5)$ & $0.031(0.9)$ & $0.071(2.1) 0.068(1.6)$ & $0.495(95.1)$ & 0.501 & 0.669 & 15.9 \\
\hline 4,4-DDE & $0.044(3.4)$ & $0.036(1.2)$ & 0.031 & 0.008 & $0.298(88.7)$ & 0.314 & 0.552 & 15.9 & $0.044(3.4)$ & $0.036(1.2)$ & $0.031(0.7)$ & $0.033(1.5) 0.060(3.5)$ & $0.452(94.1)$ & 0.384 & 0.765 & 18.6 \\
\hline 2,4-DDD & $0.044(3.3)$ & $0.036(1.3)$ & 0.031 & 0.007 & $0.332(85.6)$ & 0.315 & 0.661 & 17.6 & $0.044(3.3)$ & $0.036(1.3)$ & $0.031(0.8)$ & $0.051(1.9) 0.071(3.4)$ & $0.413(92.1)$ & 0.382 & 0.665 & 18.7 \\
\hline Endrin & $0.044(3.2)$ & $0.036(1.0)$ & $0.031(0.9)$ & 0.009 & $0.312(92.4)$ & 0.347 & 0.618 & 18.6 & $0.044(3.2)$ & $0.036(1.0)$ & $0.031(0.9)$ & $0.097(6.3) 0.062(2.6)$ & $0.402(90.8)$ & 0.381 & 0.654 & 16.9 \\
\hline Chlorfenapyl & $0.044(3.1)$ & $0.036(0.8)$ & 0.031 & 0.070 & $0.291(93.1)$ & 0.325 & 0.662 & 17.8 & $0.044(3.1)$ & $0.036(0.8)$ & $0.031(1.0)$ & $0.096(5.4) 0.045(1.6)$ & $0.350(88.4)$ & 0.504 & 0.774 & 18.3 \\
\hline 4,4-DDD & $0.044(2.1)$ & $0.036(1.0)$ & 0.031 & $0.066(0.2) 0.041$ & $0.330(94.5)$ & 0.287 & 0.665 & 17.6 & $0.044(2.1)$ & $0.036(1.0)$ & $0.031(1.1)$ & $0.071(2.1) 0.061(3.2)$ & $0.318(87.8)$ & 0.501 & 0.771 & 16.9 \\
\hline Ethion & $0.044(3.4)$ & $0.036(0.9)$ & 0.031 & $0.052(0.1) 0.068$ & $0.296(95.2)$ & 0.298 & 0.514 & 15.9 & $0.044(3.4)$ & $0.036(0.9)$ & $0.031(1.4)$ & $0.033(1.5) 0.070(3.3)$ & $0.333(92.1)$ & 0.359 & 0.659 & 18.1 \\
\hline DDT & $0.044(1.9)$ & $0.036(1.2)$ & 0.031 & $0.054(0.6) 0.052$ & $0.332(85.6)$ & 0.301 & 0.512 & 18.1 & $0.044(1.9)$ & $0.036(1.2)$ & $0.031(1.3)$ & $0.051(1.9) 0.064(2.8)$ & $0.368(91.2)$ & 0.385 & 0.662 & 18.6 \\
\hline Abamectin & $0.044(2.1)$ & $0.036(1.1)$ & $0.031(0.9)$ & $0.051(0.7) 0.037(1.7)$ & $0.301(92.1)$ & 0.305 & 0.628 & 17.6 & $0.044(2.1)$ & $0.036(1.1)$ & $0.031(0.9)$ & $0.076(2.2) 0.044(1.7)$ & $0.495(95.1)$ & 0.504 & 0.652 & 17.8 \\
\hline Acetamiprid & $0.044(2.3)$ & $0.036(1.5)$ & 0.031 & $0.041(0.5) 0.045$ & $0.317(89.5)$ & 0.321 & 0.624 & 15.9 & $0.044(2.3)$ & $0.036(1.5)$ & 0.031 & $0.071(4.4) 0.068(1.6)$ & $0.452(94.1)$ & 0.501 & 1.014 & 17.3 \\
\hline Acetochlor & $0.044(2.0)$ & $0.036(1.6)$ & 0.031 & $0.040(0.6) 0.065$ & $0.245(90.4)$ & 0.315 & 0.701 & 14.3 & $0.044(2.0)$ & $0.036(1.6)$ & 0.031 & $0.097(6.3) 0.060(3.5)$ & $0.413(92.1)$ & 0.328 & 0.665 & 19.6 \\
\hline Acrinathrin & $0.044(3.4)$ & $0.036(1.7)$ & 0.031 & $0.043(0.2) 0.048(2.2)$ & $0.298(88.7)$ & 0.311 & 0.704 & 13.6 & $0.044(3.4)$ & $0.036(1.7)$ & 0.031 & $0.096(5.4) 0.071(3.4)$ & $0.402(90.8)$ & 0.329 & 1.011 & 20.5 \\
\hline Alachlor & $0.044(3.3)$ & $0.036(1.4)$ & $0.031(0.9)$ & 0.054 & $0.332(85.6)$ & 0.314 & 0.517 & 13.5 & 0.044 & $0.036(1$ & 0.031 & $0.062(2.6)$ & $0.350(88$ & 0.385 & 0.774 & 21.5 \\
\hline Amitraz & $0.044(3.2)$ & $0.036(1.5)$ & $0.031(0.7)$ & $0.071(0.3) 0.039$ & $0.312(92.4)$ & 0.317 & 0.514 & 18.1 & $0.044(3.2)$ & $0.036(1.5)$ & $0.031(0.7)$ & $0.033(1.5) 0.045(1.6)$ & $0.318(87.8)$ & 0.384 & 0.776 & 22.5 \\
\hline Atrazine & $0.044(3.1)$ & $0.036(1.2)$ & $0.031(0.8)$ & $0.041(0.5) 0.065$ & $0.298(89.7)$ & 0.298 & 0.548 & 14.5 & $0.044(3.1)$ & $0.036(1.2)$ & $0.031(0.8)$ & $0.051(1.9) 0.061(3.2)$ & $0.333(92.1)$ & 0.507 & 0.669 & 21.7 \\
\hline Azinphos methyl & $0.044(2.1)$ & $0.036(1.3)$ & $0.031(0.9)$ & $0.040(0.6) 0.048(2.2)$ & $0.291(93.1)$ & 0.298 & 0.664 & 16.5 & $0.044(2.1)$ & $0.036(1.3)$ & $0.031(0.9)$ & $0.076(2.2) 0.070(3.3)$ & $0.368(91$. & 0.504 & 0.771 & 22.9 \\
\hline Azoxystrobine & $0.044(2.1)$ & $0.036(1.0)$ & 0.031 & $0.043(0.2) 0.048(2.2)$ & $0.332(85.6)$ & 0.337 & 0.663 & 15.7 & $0.044(2.1)$ & $0.036(1.0)$ & 0.031 & $0.071(4.4) 0.064(2.8)$ & $0.495(95$ & 0.394 & 0.778 & 21.7 \\
\hline $\begin{array}{l}\text { Bensulfuron- } \\
\text { Methyl }\end{array}$ & $0.044(2.0)$ & $0.036(0.8)$ & 0.031 & $0.054(0.4) 0.038(1.8)$ & $0.301(92.1)$ & 0.315 & 0.559 & 14.9 & $0.044(2.0)$ & $0.036(0.8)$ & 0.031 & $0.097(6.3) 0.044(1.7)$ & $0.452(94.1)$ & 0.392 & 0.668 & 20.6 \\
\hline Bentazone & $0.044(3.2)$ & $0.036(0.9)$ & 0.031 & $0.071(0.3) 0.039(1.8)$ & $0.317(89.5)$ & 0.347 & 0.557 & 17.6 & $0.044(3.2)$ & $0.036(0.9)$ & 0.031 & $0.096(5.4) 0.068(1.6)$ & $0.413(92.1)$ & 0.389 & 0.669 & 15.7 \\
\hline Beta-Cyfluthrin & $0.044(3.5)$ & $0.036(1.0)$ & 0.031 & $0.041(0.5) 0.041(2.1)$ & $0.245(90.4)$ & 0.325 & 0.621 & 18.1 & $0.044(3.5)$ & $0.036(1.0)$ & 0.031 & $0.071(2.1) 0.060(3.5)$ & $0.402(90.8)$ & 0.374 & 1.015 & 16.4 \\
\hline Bifenthrin & $0.044(1.9)$ & $0.036(0.9)$ & $0.031(0.9)$ & $0.040(0.6) 0.068(3.5)$ & $0.298(88.7)$ & 0.289 & 0.514 & 16.9 & $0.044(1.9)$ & $0.036(0.9)$ & $0.031(0.9)$ & $0.033(1.5) 0.071(3.4)$ & $0.350(88.4)$ & 0.365 & 1.016 & 18.3 \\
\hline
\end{tabular}

APPLIED ECOLOGY AND ENVIRONMENTAL RESEARCH 17(3): 6887-6916.

http://www.aloki.hu • ISSN 15891623 (Print) • ISSN 17850037 (Online)

DOI: http://dx.doi.org/10.15666/aeer/1703_68876916

๑ 2019, ALÖKI Kft., Budapest, Hungary 


\begin{tabular}{|c|c|c|c|c|c|c|c|c|c|c|c|c|c|c|c|c|}
\hline \multirow{3}{*}{ Pesticide } & \multicolumn{8}{|c|}{ Cucumber } & \multicolumn{8}{|c|}{ Grapefruit } \\
\hline & \multirow[b]{2}{*}{$\mathbf{U}_{1}$} & \multirow[b]{2}{*}{$\mathbf{U}_{2}$} & \multirow{2}{*}{$\begin{array}{c}\begin{array}{c}\mathrm{U}_{\mathrm{i}}^{\mathrm{a}} \\
(\mathrm{mg} / \mathrm{kg} \\
(\%))\end{array} \\
\mathrm{U}_{3}\end{array}$} & \multirow[b]{2}{*}{$\begin{array}{r}\mathbf{U}_{4} \\
(\mathrm{mg} / \mathrm{kg})\end{array}$} & \multirow[b]{2}{*}{$\mathbf{U}_{6}$} & \multirow[b]{2}{*}{$\mathbf{U}_{\mathbf{c}}$} & \multirow[b]{2}{*}{$\begin{array}{c}\mathbf{U}_{\mathbf{e}}^{\mathbf{b}} \\
(\mathrm{mg} / \mathrm{kg} \\
\quad) \\
\end{array}$} & \multirow[b]{2}{*}{$\begin{array}{c}\mathbf{U}_{\mathbf{e}}(\%) \\
\quad\end{array}$} & \multirow[b]{2}{*}{$\mathbf{U}_{1}$} & \multirow[b]{2}{*}{$\mathbf{U}_{2}$} & \multirow{2}{*}{$\begin{array}{c}\begin{array}{c}\mathrm{U}_{\mathrm{i}}^{\mathrm{a}} \\
(\mathrm{mg} / \mathrm{kg} \\
(\%))\end{array} \\
\mathrm{U}_{3}\end{array}$} & \multirow[b]{2}{*}{$\begin{array}{r}\mathbf{U}_{4} \\
(\mathrm{mg} / \mathrm{kg})\end{array}$} & \multirow[b]{2}{*}{$\mathbf{U}_{6}$} & \multirow[b]{2}{*}{$\mathbf{U}_{\mathbf{c}}$} & \multirow[b]{2}{*}{$\begin{array}{c}\mathbf{U}_{\mathbf{e}}^{\mathbf{b}} \\
(\mathrm{mg} / \mathrm{kg} \\
\quad) \\
\end{array}$} & \multirow[b]{2}{*}{$\begin{array}{c}\mathbf{U}_{\mathbf{e}}(\%) \\
\quad)\end{array}$} \\
\hline & & & & & & & & & & & & & & & & \\
\hline Boscalid & $0.044(2.0)$ & $0.036(1.2)$ & $0.031(0.7)$ & $0.043(0.2) 0.065(2.6)$ & 0.332 & 0.287 & 0.665 & 18.2 & $0.044(2.0)$ & $0.036(1.2)$ & 0.031 & 0.051 (1.9)0.062 (2.6) & 0.318 & 0.361 & 0.887 & 19.5 \\
\hline Bromoxynil & $0.044(3.4)$ & $0.036(1.1)$ & $0.031(0.8)$ & $0.054(0.4) 0.048(2.2)$ & 0.312 & 0.269 & 0.541 & 14.3 & 0.044 (3.4) & $0.036(1.1)$ & $0.031(0.8)$ & $0.076(2.2) 0.045(1.6)$ & 0.333 & 0.368 & 0.778 & 15.6 \\
\hline Bromuconazole & $0.044(3.3)$ & $0.036(1.5)$ & $0.031(0.9)$ & $0.071(0.3) 0.062(2.1)$ & 0.298 & 0.278 & 0.526 & 15.1 & $0.044(3.3)$ & $0.036(1.5)$ & $0.031(0.9)$ & $0.071(4.4) 0.061(3.2)$ & 0.368 & 0.501 & 0.779 & 16.2 \\
\hline Bupirimate & $0.044(3.2)$ & $0.036(1.6)$ & $0.031(1.0)$ & 0.008 (4.2)0.045 (1.8) & 0.291 & 0.259 & 0.668 & 18.3 & $0.044(3.2)$ & $0.036(1.6)$ & 0.031 & $0.097(6.3) 0.070$ ( 3.3$)$ & $0.495(95.1)$ & 0.500 & 0.765 & 19.6 \\
\hline Buprofezine & $0.044(3.1)$ & $0.036(1.7)$ & $0.031(1.4)$ & 0.007 (4.1)0.048 (2.2) & 0.332 & 0.335 & 0.662 & 14.6 & $0.044(3.1)$ & $0.036(1.7)$ & 0.031 & $0.096(5.4) 0.064(2.8)$ & 0.452 & 0.384 & 0.669 & 20.3 \\
\hline Carbaryl & $0.044(2.1)$ & $0.036(1.4)$ & $0.031(1.3)$ & ) 0.009 (3.9)0.038 (1.8) & 0.301 & 0.287 & 0.669 & 17.6 & $0.044(2.1)$ & $0.036(1.4)$ & 0.031 & $0.071(2.1) 0.044(1.7)$ & 0.413 & 0.382 & 0.765 & 21.6 \\
\hline Carbendazim & $0.044(2.3)$ & $0.036(1.5)$ & $0.031(0.9)$ & ) 0.070 (3.7)0.039 (1.8) & 0.317 & 0.298 & 0.665 & 15.9 & $0.044(2.3)$ & $0.036(1.5)$ & $0.031(0.9)$ & $0.033(1.5) 0.068(1.6)$ & 0.402 & 0.381 & 0.665 & 23.4 \\
\hline Carbofuran & $0.044(2.1)$ & $0.036(1.2)$ & $0.031(1.1)$ & ) $0.066(0.2) 0.041(2.1)$ & 0.245 & 0.301 & 0.514 & 17.6 & $0.044(2.1)$ & $0.036(1.2)$ & 0.031 & ) $0.051(1.9) 0.060(3.5)$ & 0.350 & 0.504 & 0.654 & 21.8 \\
\hline Carbosulfan & $0.044(1.9)$ & $0.036(1.3)$ & $0.031(1.4)$ & $0.052(0.1) 0.068(3.5)$ & 0.298 & 0.305 & 0.512 & 18.6 & $0.044(1.9)$ & $0.036(1.3)$ & 0.031 & ) $0.034(1.2) 0.071(3.4)$ & 0.318 & 0.501 & 0.774 & 22.8 \\
\hline Carboxin & $0.044(2.3)$ & $0.036(1.0)$ & $0.031(1.3)$ & ) $0.054(0.6) 0.052(3.4)$ & 0.291 & 0.321 & 0.628 & 17.8 & $0.044(2.3)$ & 0.036 & 0.031 & ) $0.076(2.2) 0.062(2$ & 0.333 & 0.359 & 0.771 & 21.8 \\
\hline $\begin{array}{c}\text { Carfentrazone- } \\
\text { Ethyl }\end{array}$ & $0.044(2.3)$ & $0.036(0.9)$ & $0.031(0.9)$ & $0.051(0.7) 0.037(1.7)$ & 0.338 & 0.315 & 0.624 & 17.6 & $0.044(2.3)$ & $0.036(0.9)$ & $0.031(0.9)$ & $0.071(4.4) 0.045(1.6)$ & $0.368(91.2)$ & 0.385 & 50.659 & 23.6 \\
\hline Chlofentazine & $0.044(2.1)$ & $0.036(1.0)$ & $0.031(0.7)$ & ) $0.041(0.5) 0.045(1.8)$ & 0.332 & 0.311 & 0.701 & 15.9 & $0.044(2.1)$ & $0.036(1.0)$ & 0.031 & $0.097(6.3) 0.061(3.2)$ & 0.495 & 0.504 & 0.662 & 20.8 \\
\hline Chlorfluozuron & $0.044(2.0)$ & $0.036(0.9)$ & $0.031(0.8)$ & $0.040(0.6) 0.062(2.1)$ & 0.312 & 0.314 & 0.704 & 18.1 & $0.044(2.0)$ & $0.036(0.9)$ & $0.031(0.8)$ & $0.096(5.4) 0.070$ ( 3.3$)$ & $0.452(94.1)$ & 0.501 & 0.652 & 19.6 \\
\hline Chloridazon & $0.044(3.2)$ & $0.036(1.2)$ & $0.031(0.9)$ & $0.043(0.2) 0.039$ (1.8) & 0.298 & 0.317 & 0.517 & 17.6 & $0.044(3.2)$ & $0.036(1.2)$ & 0.031 & $0.071(2.1) 0.064(2.8)$ & 0.413 & 0.328 & 3.014 & 18.9 \\
\hline $\begin{array}{l}\text { Chlormequat } \\
\text { chloride }\end{array}$ & $0.044(3.5)$ & $0.036(1.1)$ & $0.031(1.0)$ & $0.054(0.4) 0.065$ (2.6) & 0.301 & 0.298 & 0.514 & 15.9 & $0.044(3.5)$ & $0.036(1.1)$ & $0.031(1.0)$ & $0.033(1.5) 0.044(1.7)$ & 0.402 & 0.329 & 0.665 & 17.6 \\
\hline Chlorpham & $0.044(1.9)$ & $0.036(1.5)$ & $0.031(1.6)$ & $0.071(0.3) 0.048(2.2)$ & 0.317 & 0.331 & 0.548 & 14.9 & $0.044(1.9)$ & $0.036(1.5)$ & 0.031 & $0.051(1.9) 0.068(1.6)$ & 0.350 & 0.385 & 1.011 & 15.9 \\
\hline Chlorpyrifos ethyl & $0.044(2.0)$ & $0.036(1.6)$ & $0.031(1.0)$ & ) $0.041(0.5) 0.045(1.8)$ & 0.245 & 0.289 & 0.514 & 17.5 & $0.044(2.0)$ & $0.036(1.6)$ & $0.031(1.0)$ & $0.076(2.2) 0.060(3.5)$ & 0.318 & 0.384 & 0.774 & 19.8 \\
\hline $\begin{array}{l}\text { Chlorpyriphos } \\
\text { Methyl }\end{array}$ & $0.044(3.4)$ & $0.036(1.7)$ & $0.031(1.1)$ & ) $0.040(0.6) 0.048(2.2)$ & 0.298 & 0.287 & 0.665 & 15.3 & $0.044(3.4)$ & $0.036(1.7)$ & 0.031 & ) 0.071 (4.4)0.071 (3.4) & 0.333 & 0.394 & 0.776 & 18.7 \\
\hline Chlorsulfuron & $0.044(3.3)$ & $0.036(1.4)$ & $0.031(1.4)$ & $0.043(0.2) 0.038(1.8)$ & 0.291 & 0.269 & 0.541 & 16.1 & $0.044(3.3)$ & $0.036(1.4)$ & 0.031 & ) $0.097(6.3) 0.062(2.6)$ & $0.368(91.2)$ & 0.392 & 0.669 & 19.3 \\
\hline Clethodim & $0.044(3.2)$ & $0.036(1.5)$ & $0.031(1.3)$ & ) $0.054(0.4) 0.039$ (1.8) & 0.298 & 0.278 & 0.526 & 14.7 & $0.044(3.2)$ & $0.036(1.5)$ & 0.031 & $0.096(5.4) 0.045(1.6)$ & 0.495 & 0.389 & 0.771 & 17.6 \\
\hline
\end{tabular}

APPLIED ECOLOGY AND ENVIRONMENTAL RESEARCH 17(3): 6887-6916.

http://www.aloki.hu • ISSN 15891623 (Print) • ISSN 17850037 (Online)

DOI: http://dx.doi.org/10.15666/aeer/1703_68876916

(c) 2019, ALÖKI Kft., Budapest, Hungary 


\begin{tabular}{|c|c|c|c|c|c|c|c|c|c|c|c|c|c|c|c|c|}
\hline \multirow{3}{*}{ Pesticide } & \multicolumn{8}{|c|}{ Cucumber } & \multicolumn{8}{|c|}{ Grapefruit } \\
\hline & \multirow[b]{2}{*}{$\mathbf{U}_{1}$} & \multirow[b]{2}{*}{$\mathbf{U}_{2}$} & \multirow{2}{*}{$\begin{array}{c}\begin{array}{c}\mathrm{U}_{\mathrm{i}}^{\mathrm{a}} \\
(\mathrm{mg} / \mathrm{kg} \\
(\%))\end{array} \\
\mathrm{U}_{3}\end{array}$} & \multirow[b]{2}{*}{$\begin{array}{l}\mathbf{U}_{4} \\
\mathrm{~kg})\end{array}$} & \multirow[b]{2}{*}{$\mathbf{U}_{6}$} & \multirow[b]{2}{*}{$\mathbf{U}_{\mathbf{c}}$} & \multirow[b]{2}{*}{$\begin{array}{c}\mathbf{U}_{\mathbf{e}}^{\mathbf{b}} \\
(\mathrm{mg} / \mathrm{kg} \\
)\end{array}$} & \multirow[b]{2}{*}{$\begin{array}{c}\mathbf{U}_{\mathrm{e}}(\% \\
)\end{array}$} & \multirow[b]{2}{*}{$\mathbf{U}_{1}$} & \multirow[b]{2}{*}{$\mathbf{U}_{2}$} & \multirow{2}{*}{$\begin{array}{c}\begin{array}{c}\mathrm{U}_{\mathrm{i}}^{\mathrm{a}} \\
(\mathrm{mg} / \mathrm{kg} \\
(\%))\end{array} \\
\mathrm{U}_{3}\end{array}$} & \multirow[b]{2}{*}{$\begin{array}{r}\mathbf{U}_{\mathbf{4}} \\
(\mathrm{mg} / \mathrm{kg}) \\
\end{array}$} & \multirow[b]{2}{*}{$\mathbf{U}_{6}$} & \multirow[b]{2}{*}{$\mathbf{U}_{\mathrm{c}}$} & \multirow[b]{2}{*}{$\begin{array}{c}\mathbf{U}_{\mathbf{e}}^{\mathbf{b}} \\
(\mathrm{mg} / \mathrm{kg} \\
)\end{array}$} & \multirow[b]{2}{*}{$\begin{array}{c}\mathrm{U}_{\mathrm{e}}(\%) \\
)\end{array}$} \\
\hline & & & & & & & & & & & & & & & & \\
\hline $\begin{array}{l}\text { Clodinafop- } \\
\text { Propargyl }\end{array}$ & $0.044(3.1)$ & 0.036 & $0.031(0.9)$ & $0.071(0.3) 0.041(2.1)$ & 0.332 & 0.259 & 0.668 & 15.2 & $0.044(3.1)$ & $0.036(1.2)$ & $0.031(0.9)$ & 0.071 & 0.452 & 0.374 & 0.778 & 20.1 \\
\hline Clothianidin & $0.044(2.1)$ & $0.036(1.3)$ & 0.031 & $0.008(4.2) 0.068(3.5)$ & $0.312(92.4)$ & 0.332 & 0.662 & 12.5 & $0.044(2.1)$ & $0.036(1.3)$ & 0.031 & $0.033(1.5) 0.070$ & $0.413(92.1)$ & 0.365 & 0.668 & 21.5 \\
\hline Cyclanilide & $0.044(2.3)$ & $0.036(1.0)$ & 0.031 & $0.007(4.1) 0.065(2.6)$ & 0.298 & 0.287 & 0.669 & 13.6 & $0.044(2.3)$ & $0.036(1.0)$ & 0.031 & $0.051(1.9) 0.064$ & $0.402(90.8)$ & 0.361 & 0.669 & 23.4 \\
\hline Cycloate & $0.044(2.1)$ & $0.036(0.8)$ & 0.031 & $0.009(3.9) 0.048(2.2)$ & 0.301 & 0.298 & 0.665 & 18.1 & $0.044(2.1)$ & $0.036(0.8)$ & 0.031 & $0.034(1.2) 0.044$ & 0.350 & 0.368 & 0.664 & 20.9 \\
\hline Cyhalofop-butyl & $0.044(1.9)$ & $0.036(0.9)$ & $0.031(0.9)$ & $0.070(3.7) 0.039(1.8)$ & 0.317 & 0.301 & 0.514 & 14.8 & $0.044(1.9)$ & $0.036(0.9)$ & $0.031(0.9)$ & $0.076(2.2) 0.068$ & 0.318 & 0.498 & 0.774 & 21.7 \\
\hline Cymoxanyl & $0.044(3.2)$ & $0.036(1.0)$ & 0.031 & $0.066(0.2) 0.062(2.1)$ & 0.245 & 0.305 & 0.512 & 17.5 & $0.044(3.2)$ & $0.036(1.0)$ & 0.031 & $0.071(4.4) 0.060$ & 0.333 & 0.465 & 0.778 & 19.8 \\
\hline Cypermethrin & $0.044(3.1)$ & $0.036(0.9)$ & $0.031(0.8)$ & $0.052(0.1) 0.048(2.2)$ & 0.298 & 0.321 & 0.628 & 17.6 & $0.044(3.1)$ & $0.036(0.9)$ & $0.031(0.8)$ & 0.097 & 0.368 & 0.395 & 0.779 & 19.5 \\
\hline Cyproconazole & $0.044(2.8)$ & $0.036(1.2)$ & $0.031(0.9)$ & $0.054(0.6) 0.038(1.8)$ & 0.337 & 0.315 & 0.624 & 12.5 & $0.044(2.8)$ & $0.036(1.2)$ & $0.031(0.9)$ & $0.096(5.4) 0.062$ & 0.495 & 0.401 & 1.010 & 19.1 \\
\hline Cyprodinile & $0.044(2.0)$ & $0.036(1.1)$ & 0.031 & $0.051(0.7) 0.039(1.8)$ & 0.298 & 0.311 & 0.701 & 14.8 & $0.044(2.0)$ & $0.036(1.1)$ & $0.031(1.0)$ & $0.071(2.1) 0.045$ & $0.452(94.1)$ & 0.425 & 1.011 & 18.7 \\
\hline Dazomet & $0.044(3.4)$ & $0.036(1.5)$ & 0.031 & $0.041(0.5) 0.041(2.1)$ & 0.332 & 0.314 & 0.704 & 17.9 & $0.044(3.4)$ & $0.036(1.5)$ & 0.031 & $0.033(1.5) 0.061$ & $0.413(92.1)$ & 0.384 & 0.998 & 19.3 \\
\hline Deltamethrin & $0.044(3.3)$ & $0.036(1.6)$ & 0.031 & $0.040(0.6) 0.068(3.5)$ & 0.312 & 0.317 & 0.517 & 18.6 & $0.044(3.3)$ & $0.036(1.6)$ & $0.031(1.4)$ & $0.051(1.9) 0.070$ & $0.402(90.8)$ & 0.382 & 0.884 & 16.4 \\
\hline Diafenthiuron & $0.044(3.2)$ & $0.036(1.7)$ & 0.031 & $0.043(0.2) 0.039(1.8)$ & 0.298 & 0.298 & 0.514 & 16.5 & $0.044(3.2)$ & $0.036(1.7)$ & 0.031 & $0.076(2.2) 0.064$ & $0.350(88.4)$ & 0.381 & 0.765 & 19.1 \\
\hline Diazinon & $0.044(3.1)$ & $0.036(1.4)$ & $0.031(0.9)$ & $0.054(0.4) 0.065(2.6)$ & 0.301 & 0.289 & 0.548 & 15.4 & $0.044(3.1)$ & $0.036(1.4)$ & $0.031(0.9)$ & $0.071(4.4) 0.044$ & $0.318(87.8)$ & 0.504 & 0.665 & 18.4 \\
\hline Dicamba & $0.044(2.1)$ & $0.036(1.5)$ & $0.031(0.7)$ & $0.071(0.3) 0.048(2.2)$ & 0.317 & 0.287 & 0.612 & 15.8 & $0.044(2.1)$ & $0.036(1.5)$ & $0.031(0.7)$ & $0.097(6.3) 0.068$ & $0.333(92.1)$ & 0.501 & 0.654 & 19.6 \\
\hline Dichlofluanid & $0.044(2.1)$ & $0.036(1.2)$ & $0.031(0.8)$ & $0.041(0.5) 0.048(2.2)$ & 0.245 & 0.269 & 0.514 & 15.9 & $0.044(2.1)$ & $0.036(1.2)$ & $0.031(0.8)$ & 0.096 & $0.368(91.2)$ & 0.359 & 0.774 & 18.9 \\
\hline Dichlorvos & $0.044(2.0)$ & 0.036 & $0.031(0.9)$ & $0.040(0.6) 0.038(1.8)$ & 0.298 & 0.278 & 0.665 & 17.6 & $0.044(2.0)$ & $0.036(1.3)$ & $0.031(0.9)$ & 0.071 & $0.495(95.1)$ & 0.385 & 0.771 & 15.8 \\
\hline Diclofop Methyl & $0.044(3.2)$ & $0.036(1.0)$ & 0.031 & $0.043(0.2) 0.039(1.8)$ & 0.332 & 0.259 & 0.541 & 18.6 & $0.044(3.2)$ & $0.036(1.0)$ & $0.031(1.0)$ & $0.033(1.5) 0.062$ & $0.452(94.1)$ & 0.504 & 0.659 & 19.6 \\
\hline Diethofencarb & $0.044(3.5)$ & $0.036(1.6)$ & 0.031 & $0.054(0.4) 0.041(2.1)$ & $0.312(92.4)$ & 0.315 & 0.526 & 17.8 & $0.044(3.5)$ & $0.036(1.6)$ & 0.031 & $0.051(1.9) 0.045$ & $0.413(92.1)$ & 0.501 & 0.662 & 20.1 \\
\hline Difenoconazole & $0.044(1.9)$ & $0.036(1.5)$ & 0.031 & $0.071(0.3) 0.068(3.5)$ & 0.298 & 0.347 & 0.668 & 17.6 & $0.044(1.9)$ & $0.036(1.5)$ & 0.031 & $0.034(1.2) 0.061$ & $0.402(90.8)$ & 0.328 & 0.652 & 15.7 \\
\hline Diflubenzuron & $0.044(3.2)$ & 0.036 & $0.031(0.9)$ & $0.007(4.1) 0.065(2.6)$ & 0.291 & 0.325 & 0.662 & 15.9 & $0.044(3.2)$ & $0.036(1.0)$ & $0.031(0.9)$ & $0.076(2.2) 0.070$ & $0.350(88.4)$ & 0.329 & 1.014 & 16.4 \\
\hline Dimethenamid & $0.044(3.1)$ & $0.036(0.9)$ & 0.031 & $0.009(3.9) 0.048(2.2)$ & 0.337 & 0.333 & 0.669 & 18.1 & $0.044(3.1)$ & $0.036(0.9)$ & 0.031 & 0.071 & $0.318(87.8)$ & 0.385 & 0.665 & 18.3 \\
\hline
\end{tabular}

APPLIED ECOLOGY AND ENVIRONMENTAL RESEARCH 17(3): 6887-6916.

http://www.aloki.hu • ISSN 15891623 (Print) • ISSN 17850037 (Online)

DOI: http://dx.doi.org/10.15666/aeer/1703_68876916

(c) 2019, ALÖKI Kft., Budapest, Hungary 


\begin{tabular}{|c|c|c|c|c|c|c|c|c|c|c|c|c|c|c|c|c|}
\hline \multirow{3}{*}{ Pesticide } & \multicolumn{8}{|c|}{ Cucumber } & \multicolumn{8}{|c|}{ Grapefruit } \\
\hline & \multirow[b]{2}{*}{$\mathbf{U}_{1}$} & \multirow[b]{2}{*}{$\mathbf{U}_{2}$} & \multirow{2}{*}{$\begin{array}{c}\begin{array}{c}\mathrm{U}_{\mathrm{i}}^{\mathrm{a}} \\
(\mathrm{mg} / \mathrm{kg} \\
(\%)\end{array} \\
\mathrm{U}_{3}\end{array}$} & \multirow[b]{2}{*}{$\begin{array}{r}\mathbf{U}_{4} \\
(\mathrm{mg} / \mathrm{kg})\end{array}$} & \multirow[b]{2}{*}{$\mathbf{U}_{6}$} & \multirow[b]{2}{*}{$\mathbf{U}_{\mathrm{c}}$} & \multirow[b]{2}{*}{$\begin{array}{c}\mathbf{U}_{\mathbf{e}}^{\mathbf{b}} \\
(\mathrm{mg} / \mathrm{kg} \\
)\end{array}$} & \multirow[b]{2}{*}{$\begin{array}{c}\mathbf{U}_{\mathrm{e}}(\%) \\
\quad)\end{array}$} & \multirow[b]{2}{*}{$\mathbf{U}_{1}$} & \multirow[b]{2}{*}{$\mathbf{U}_{2}$} & \multirow{2}{*}{$\begin{array}{c}\begin{array}{c}\mathrm{U}_{\mathrm{i}}^{\mathrm{a}} \\
(\mathrm{mg} / \mathrm{kg} \\
(\%))\end{array} \\
\mathrm{U}_{3} \\
\end{array}$} & \multirow[b]{2}{*}{$\begin{array}{r}\mathbf{U}_{\mathbf{4}} \\
(\mathrm{mg} / \mathrm{kg}) \\
\end{array}$} & \multirow[b]{2}{*}{$\mathbf{U}_{6}$} & \multirow[b]{2}{*}{$\mathbf{U}_{\mathbf{c}}$} & \multirow[b]{2}{*}{$\begin{array}{c}\mathbf{U}_{\mathbf{e}}^{\mathbf{b}^{\mathbf{b}}} \\
(\mathrm{mg} / \mathrm{kg} \\
)\end{array}$} & \multirow[b]{2}{*}{$\begin{array}{c}\mathbf{U}_{\mathbf{e}}(\%) \\
)\end{array}$} \\
\hline & & & & & & & & & & & & & & & & \\
\hline Dimethoate & $0.044(2.8)$ & 0.036 & $0.031(1.4)$ & 0.070 & $0.298(89.7)$ & 0.287 & 0.618 & 17.6 & $0.044(2.8)$ & $0.036(1.2)$ & $0.031(1.4)$ & $0.097(6.3) 0.044(1.7)$ & ) $0.333(9$ & 0.384 & 1.011 & 19.5 \\
\hline Dimethomorph & $0.044(3.4)$ & 0.036 & $0.031(1.3)$ & $0.066(0.2) 0.062(2.1)$ & $0.301(92.1)$ & 0.298 & 0.662 & 15.9 & $0.044(3.4)$ & $0.036(1.1)$ & $0.031(1.3)$ & $0.096(5.4) 0.068(1.6)$ & $0.368(91.2)$ & 2) 0.394 & 0.774 & 15.6 \\
\hline Diniconazole & $0.044(3.3)$ & $0.036(1.5)$ & $0.031(0.9)$ & $0.052(0.1) 0.045$ & $0.317(89.5)$ & 0.301 & 0.665 & 15.6 & $0.044(3.3)$ & $0.036(1.5)$ & $0.031(0.9)$ & $0.071(2.1) 0.060(3.5)$ & $0.495(95.1)$ & ) 0.392 & 20.776 & 16.2 \\
\hline Dinocap & $0.044(3.2)$ & $0.036(1.6)$ & $0.031(0.7)$ & $0.054(0.6) 0.048(2.2)$ & $0.245(90.4)$ & 0.305 & 0.514 & 15.8 & $0.044(3.2)$ & $0.036(1.6)$ & $0.031(0.7)$ & $0.033(1.5) 0.071(3.4)$ & $0.452(94.1)$ & ) 0.389 & 0.669 & 19.6 \\
\hline Dioxathion & $0.044(3.1)$ & $0.036(1.7)$ & $0.031(0.8)$ & $0.051(0.7) 0.038(1.8)$ & $0.298(88.7)$ & 0.321 & 0.512 & 17.4 & $0.044(3.1)$ & $0.036(1.7)$ & $0.031(0.8)$ & $0.051(1.9) 0.062(2.6)$ & ) $0.413(92.1)$ & ) 0.374 & 0.771 & 20.3 \\
\hline Diphenamid & $0.044(2.1)$ & $0.036(1.4)$ & $0.031(0.9)$ & $0.041(0.5) 0.039(1.8)$ & $0.332(85.6)$ & 0.315 & 0.628 & 16.5 & $0.044(2.1)$ & $0.036(1.4)$ & $0.031(0.9)$ & $0.076(2.2) 0.045(1.6)$ & $0.402(90.8)$ & 0.365 & 0.778 & 21.6 \\
\hline Dithianon & $0.044(2.3)$ & $0.036(1.5)$ & $0.031(1.0)$ & $0.040(0.6) 0.041(2.1)$ & $0.312(92.4)$ & 0.311 & 0.624 & 13.4 & $0.044(2.3)$ & $0.036(1.5)$ & $0.031(1.0)$ & $0.071(4.4) 0.061(3.2)$ & $0.350(88.4)$ & ) 0.361 & 0.668 & 23.4 \\
\hline Diuron & $0.044(2.1)$ & $0.036(1.2)$ & $0.031(1.5)$ & $0.043(0.2) 0.068(3.5)$ & $0.298(89.7)$ & 0.314 & 0.701 & 15.2 & $0.044(2.1)$ & $0.036(1.2)$ & $0.031(1.5)$ & $0.097(6.3) 0.070(3.3)$ & 0.318 (87.8) & ) 0.368 & 0.669 & 21.8 \\
\hline Epoxyconazole & $0.044(2.0)$ & $0.036(1.3)$ & $0.031(1.6)$ & $0.054(0.4) 0.052$ & $0.337(92.6)$ & 0.317 & 0.704 & 14.2 & $0.044(2.0)$ & $0.036(1.3)$ & $0.031(1.6)$ & $0.096(5.4) 0.064(2.8)$ & $0.333(92$. & 0.432 & 0.774 & 22.8 \\
\hline EPTC & $0.044(3.2)$ & $0.036(1.0)$ & 0.031 & $0.071(0.3) 0.037(1.7)$ & $0.332(87.4)$ & 0.298 & 0.517 & 18.1 & $0.044(3.2)$ & $0.036(1.0)$ & 0.031 & $0.071(2.1) 0.044(1.7)$ & $0.368(91.2)$ & ) 0.425 & 0.885 & 21.8 \\
\hline Esfenvalerate & $0.044(3.5)$ & $0.036(1.4)$ & 0.031 & $0.041(0.5) 0.045$ & $0.301(92.1)$ & 0.289 & 0.514 & 15.4 & $0.044(3.5)$ & $0.036(1.4)$ & 0.031 & $0.033(1.5) 0.068(1.6)$ & $0.495(95.1)$ & ) 0.421 & 0.668 & 23.6 \\
\hline Ethalfluralin & $0.044(1.9)$ & $0.036(1.0)$ & 0.031 & $0.040(0.6) 0.065$ & $0.317(89.5)$ & 0.287 & 0.548 & 16.4 & $0.044(1.9)$ & $0.036(1.0)$ & 0.031 & $0.051(1.9) 0.060(3.5)$ & (0 & ) 0.384 & 0.662 & 20.8 \\
\hline Ethiofencarb & $0.044(2.0)$ & $0.036(0.9)$ & 0.031 & $0.043(0.2) 0.048(2.2)$ & $0.245(90.4)$ & 0.269 & 0.621 & 15.9 & $0.044(2.0)$ & $0.036(0.9)$ & 0.031 & $0.050(1.7) 0.071(3.4)$ & $0.413(92.1)$ & ) 0.382 & 0.775 & 19.6 \\
\hline Ethofumasate & $0.044(3.4)$ & $0.036(1.2)$ & $0.031(0.9)$ & $0.054(0.4) 0.062(2.1)$ & $0.298(88.7)$ & 0.278 & 0.514 & 17.6 & $0.044(3.4)$ & $0.036(1.2)$ & $0.031(0.9)$ & $0.034(1.2) 0.062(2.6)$ & $0.402(90.8)$ & ) 0.381 & 0.771 & 18.9 \\
\hline Ethoprophos & $0.044(3.3)$ & $0.036(1.1)$ & 0.031 & $0.071(0.3) 0.039(1.8)$ & $0.291(93.1)$ & 0.259 & 0.665 & 18.6 & $0.044(3.3)$ & $0.036(1.1)$ & 0.031 & $0.076(2.2) 0.045(1.6)$ & $0.350(88.4)$ & ) 0.504 & 0.770 & 17.6 \\
\hline Etofenprox & $0.044(3.2)$ & $0.036(1.5)$ & 0.031 & $0.023(0.7) 0.065$ & $0.298(89.7)$ & 0.287 & 0.541 & 17.8 & $0.044(3.2)$ & $0.036(1.5)$ & 0.031 & $0.071(4.4) 0.061(3.2)$ & $0.318(87.8)$ & 0.501 & 0.776 & 15.9 \\
\hline Etoxazole & $0.044(3.1)$ & $0.036(1.6)$ & 0.031 & $0.072(4.3) 0.048(2.2)$ & $0.337(92.6)$ & 0.298 & 0.526 & 17.6 & $0.044(3.1)$ & 0.036 & 0.031 & $0.097(6.3) 0.070(3.3)$ & $0.333(92.1)$ & 0.359 & 0.778 & 20.1 \\
\hline Famoxadone & $0.044(2.1)$ & $0.036(1.7)$ & $0.031(0.9)$ & $0.008(4.2) 0.048(2.2)$ & $0.298(89.7)$ & 0.301 & 0.668 & 15.9 & $0.044(2.1)$ & $0.036(1.7)$ & $0.031(0.9)$ & $0.096(5.4) 0.064(2.8)$ & $0.368(91.2)$ & 0.385 & 0.776 & 21.8 \\
\hline Fenamidone & $0.044(3.2)$ & $0.036(1.4)$ & $0.031(0.7)$ & $0.007(4.1) 0.038(1.8)$ & $0.332(85.6)$ & 0.305 & 0.662 & 18.1 & $0.044(3.2)$ & 0.036 & $0.031(0.7)$ & $0.071(2.1) 0.044(1.7)$ & $0.495(95$ & 0.504 & 0.765 & 22.6 \\
\hline $\begin{array}{l}\text { Fenamiphos } \\
\quad \text { (sum) }\end{array}$ & $0.044(3.1)$ & $0.036(1.5)$ & $0.031(0$ & $0.009(3.9) 0.039(1.8)$ & $0.312(92.4)$ & 0.321 & 0.669 & 17.6 & $0.044(3.1)$ & $0.036(1.5)$ & $0.031(0.8)$ & $0.033(1.5) 0.068(1.6)$ & $0.452(94.1)$ & 0.501 & 0.665 & 19.3 \\
\hline Fenarimol & $0.044(2.8)$ & $0.036(1.2)$ & $0.031(0.9)$ & 0.070 & $0.298(89.7)$ & 0.315 & 0.662 & 15.9 & $0.044(2.8)$ & 0.036 & $0.031(0.9)$ & $0.051(1.9) 0.060(3.5)$ & $0.413(92.1)$ & 0.328 & 0.654 & 18.9 \\
\hline Fenazaquin & $0.044(3.1)$ & $0.036(1.3)$ & 0.031 & $0.066(0.2) 0.068(3.5)$ & $0.301(92.1)$ & 0.311 & 0.665 & 16.5 & $0.044(3.1)$ & 0.036 & $0.031(1.0)$ & $0.076(2.2) 0.071(3.4)$ & ) $0.402(90.8)$ & 0.329 & 0.774 & 15.6 \\
\hline
\end{tabular}

APPLIED ECOLOGY AND ENVIRONMENTAL RESEARCH 17(3): 6887-6916.

http://www.aloki.hu • ISSN 15891623 (Print) • ISSN 17850037 (Online)

DOI: http://dx.doi.org/10.15666/aeer/1703_68876916

(c) 2019, ALÖKI Kft., Budapest, Hungary 


\begin{tabular}{|c|c|c|c|c|c|c|c|c|c|c|c|c|c|c|c|c|}
\hline \multirow{3}{*}{ Pesticide } & \multicolumn{8}{|c|}{ Cucumber } & \multicolumn{8}{|c|}{ Grapefruit } \\
\hline & \multirow[b]{2}{*}{$\mathbf{U}_{1}$} & \multirow[b]{2}{*}{$\mathbf{U}_{2}$} & \multirow{2}{*}{\multicolumn{2}{|c|}{ 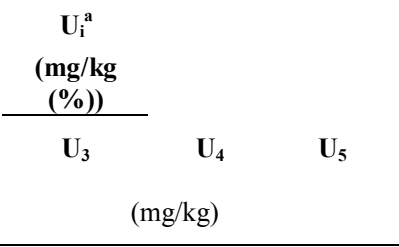 }} & \multirow[b]{2}{*}{$\mathbf{U}_{6}$} & \multirow[b]{2}{*}{$\mathbf{U}_{\mathbf{c}}$} & \multirow[b]{2}{*}{$\begin{array}{c}\mathbf{U}_{\mathbf{e}}^{\mathbf{b}} \\
(\mathrm{mg} / \mathrm{kg} \\
)\end{array}$} & \multirow[b]{2}{*}{$\begin{array}{c}\mathbf{U}_{\mathbf{e}}(\% \\
)\end{array}$} & \multirow[b]{2}{*}{$\mathbf{U}_{1}$} & \multirow[b]{2}{*}{$\mathbf{U}_{2}$} & \multirow{2}{*}{$\begin{array}{c}\begin{array}{c}\mathrm{U}_{\mathrm{i}}^{\mathrm{a}} \\
(\mathrm{mg} / \mathrm{kg} \\
(\%))\end{array} \\
\mathrm{U}_{3}\end{array}$} & \multirow[b]{2}{*}{$\begin{array}{r}\mathbf{U}_{4} \\
(\mathrm{mg} / \mathrm{kg})\end{array}$} & \multirow[b]{2}{*}{$\mathbf{U}_{6}$} & \multirow[b]{2}{*}{$\mathbf{U}_{\mathbf{c}}$} & \multirow[b]{2}{*}{$\begin{array}{c}\mathbf{U}_{\mathbf{e}}^{\mathbf{b}} \\
(\mathrm{mg} / \mathrm{kg} \\
)\end{array}$} & \multirow[b]{2}{*}{$\begin{array}{c}\mathbf{U}_{\mathrm{e}}(\%) \\
\mathbf{g}\end{array}$} \\
\hline & & & & & & & & & & & & & & & & \\
\hline Fenbuconazole & $0.044(3.3)$ & 0.036 & $0.031(1.1)$ & ) $0.052(0.1) 0.039(1.8)$ & 0.317 & 0.314 & 0.514 & 15.3 & $0.044(3.3)$ & $0.036(1.0)$ & 0.031 & $0.071(4.4) 0.062(2.6)$ & 0.350 & 0.385 & 0.771 & 16.2 \\
\hline $\begin{array}{c}\text { Fenoxaprop-P- } \\
\text { Ethyl }\end{array}$ & $0.044(2.3)$ & $0.036(0.9)$ & ) $0.031(1.4)$ & $0.054(0.6) 0.065(2.6)$ & 0.245 & 0.317 & 0.512 & 15.9 & $0.044(2.3)$ & $0.036(0.9)$ & 0.031 & $0.097(6.3) 0.045(1.6)$ & 0.318 & 0.384 & 0.659 & 19.6 \\
\hline Fenoxycarb & $0.044(2.1)$ & $0.036(0.8)$ & $0.031(1.3)$ & ) $0.051(0.7) 0.048(2.2)$ & 0.298 & 0.298 & 0.628 & 14.8 & $0.044(2.1)$ & $0.036(0.8)$ & 0.031 & $0.096(5.4) 0.061(3.2)$ & 0.333 & 0.394 & 0.662 & 20.3 \\
\hline Fenpropathrin & $0.044(2.0)$ & 0.036 & $0.031(0.9)$ & ) $0.041(0.5) 0.048(2.2)$ & 0.291 & 0.335 & 0.624 & 18.1 & $0.044(2.0)$ & $0.036(1.6)$ & $0.031(0.9)$ & $0.071(2.1) 0.070$ & $0.368(91.2)$ & 0.392 & 0.652 & 21.6 \\
\hline Fenpyroximate & $0.044(3.2)$ & 0.036 & $0.031(0.7)$ & $0.040(0.6) 0.038(1.8)$ & 0.298 & 0.289 & 0.701 & 16.9 & $0.044(3.2)$ & $0.036(1.0)$ & 0.031 & $0.033(1.5) 0.064(2.8)$ & 0.495 & 0.389 & 1.014 & 18.9 \\
\hline Fenthion & $0.044(3.5)$ & $0.036(0.9)$ & $0.031(0.8)$ & $0.043(0.2) 0.039(1.8)$ & 0.285 & 0.287 & 0.704 & 15.8 & $0.044(3.5)$ & $0.036(0.9)$ & $0.031(0.8)$ & $0.051(1.9) 0.044(1.7)$ & $0.452(94.1)$ & 0.374 & 0.665 & 17.8 \\
\hline Fenvalerate & $0.044(1.9)$ & $0.036(1.2)$ & $0.031(0.9)$ & ) $0.054(0.4) 0.041(2.1)$ & 0.331 & 0.269 & 0.517 & 17.5 & $0.044(1.9)$ & $0.036(1.2)$ & $0.031(0.9)$ & $0.076(2.2) 0.068(1.6)$ & $0.413(92.1)$ & 0.365 & 1.011 & 16.9 \\
\hline Fipronil & $0.044(2.0)$ & 0.036 & $0.031(1.0)$ & ) $0.071(0.3) 0.068(3.5)$ & 0.337 & 0.278 & 0.514 & 15.8 & $0.044(2.0)$ & $0.036(1.1)$ & $0.031(1.0)$ & $0.071(4.4) 0.060$ & 0.402 & 0.361 & 0.774 & 20.1 \\
\hline Fluazifop-P-Butyl & $0.044(3.4)$ & $0.036(1.5)$ & $0.031(1.4)$ & $0.041(0.5) 0.062(2.1)$ & 0.298 & 0.259 & 0.548 & 16.4 & $0.044(3.4)$ & $0.036(1.5)$ & 0.031 & $0.097(6.3) 0.071(3.4)$ & 0.350 & 0.368 & 0.776 & 23.4 \\
\hline Fluazinam & $0.044(3.3)$ & $0.036(1.6)$ & $0.031(1.3)$ & ) $0.040(0.6) 0.039(1.8)$ & 0.291 & 0.315 & 0.587 & 15.8 & $0.044(3.3)$ & $0.036(1.6)$ & 0.031 & $0.096(5.4) 0.062(2.6)$ & 0.318 & 0.401 & 0.669 & 20.4 \\
\hline Fludioxynil & $0.044(3.2)$ & $0.036(1.7)$ & $0.031(0.9)$ & $0.043(0.2) 0.045(1.8)$ & 0.332 & 0.347 & 0.514 & 15.9 & $0.044(3.2)$ & $0.036(1.7)$ & $0.031(0.9)$ & $0.071(2.1) 0.045(1.6)$ & 0.333 & 0.384 & 0.771 & 21.8 \\
\hline Flufenoxuron & $0.044(3.1)$ & $0.036(1.4)$ & $0.031(1.1)$ & ) $0.054(0.4) 0.048(2.2)$ & 0.312 & 0.325 & 0.665 & 18.1 & $0.044(3.1)$ & $0.036(1.4)$ & 0.031 & $0.033(1.5) 0.061(3.2)$ & 0.368 & 0.382 & 0.778 & 19.8 \\
\hline Flurasulam & $0.044(2.1)$ & $0.036(1.5)$ & ) $0.031(1.4)$ & $0.071(0.3) 0.038(1.8)$ & 0.298 & 0.287 & 0.541 & 15.9 & $0.044(2.1)$ & $0.036(1.5)$ & 0.031 & $0.051(1.9) 0.070$ & $0.495(95.1)$ & 0.381 & 0.668 & 18.9 \\
\hline Flurochloridone & $0.044(3.2)$ & $0.036(1.2)$ & ) $0.031(1.3)$ & ) $0.008(4.2) 0.039(1.8)$ & 0.301 & 0.298 & 0.526 & 17.6 & $0.044(3.2)$ & $0.036(1.2)$ & 0.031 & $0.096(5.4) 0.064(2.8)$ & 0.452 & 0.504 & 0.669 & 15.7 \\
\hline Flusilazole & $0.044(3.1)$ & $0.036(1.3)$ & $0.031(0.9)$ & ) 0.007 (4.1)0.041 (2.1) & 0.317 & 0.301 & 0.668 & 18.6 & $0.044(3.1)$ & $0.036(1.3)$ & $0.031(0.9)$ & $0.071(2.1) 0.044(1.7)$ & 0.413 & 0.501 & 0.770 & 16.4 \\
\hline Flutriafol & $0.044(2.8)$ & $0.036(1.0)$ & $0.031(0.7)$ & 0.009 (3.9)0.068 (3.5) & 0.245 & 0.305 & 0.662 & 17.8 & $0.044(2.8)$ & $0.036(1.0)$ & $0.031(0.7)$ & $0.033(1.5) 0.068(1.6)$ & 0.402 & 0.359 & 0.776 & 18.3 \\
\hline Foramsulfuron & $0.044(2.3)$ & $0.036(0.9)$ & $0.031(0.8)$ & $0.070(3.7) 0.039(1.8)$ & 0.298 & 0.321 & 0.669 & 17.6 & $0.044(2.3)$ & $0.036(0.9)$ & 0.031 & $0.051(1.9) 0.060$ & 0.350 & 0.385 & 0.778 & 19.5 \\
\hline Formatanate & $0.044(2.1)$ & $0.036(0.8)$ & $0.031(0.9)$ & $0.066(0.2) 0.065(2.6)$ & 0.285 & 0.315 & 0.665 & 15.9 & $0.044(2.1)$ & $0.036(0.8)$ & 0.031 & $0.076(2.2) 0.071(3.4)$ & 0.318 & 0.504 & 0.776 & 15.6 \\
\hline Fosthiazate & $0.044(2.0)$ & $0.036(1.6)$ & ) $0.031(1.0)$ & $0.052(0.1) 0.048(2.2)$ & 0.337 & 0.311 & 0.663 & 18.1 & $0.044(2.0)$ & $0.036(1.6)$ & $0.031(1.0)$ & $0.071(4.4) 0.062(2.6)$ & 0.333 & 0.501 & 0.765 & 16.2 \\
\hline Furathiocarb & $0.044(3.2)$ & $0.036(1.0)$ & $0.031(0.9)$ & $0.054(0.6) 0.048(2.2)$ & 0.298 & 0.314 & 0.664 & 17.6 & $0.044(3.2)$ & $0.036(1.0)$ & $0.031(0.9)$ & $0.097(6.3) 0.045(1.6)$ & 0.368 & 0.328 & 0.998 & 19.6 \\
\hline Giberellic acid & $0.044(3.5)$ & $0.036(0.9)$ & $0.031(0.8)$ & $0.051(0.7) 0.038(1.8)$ & 0.332 & 0.317 & 0.521 & 15.9 & $0.044(3.5)$ & $0.036(0.9)$ & 0.031 & $0.096(5.4) 0.061$ & 0.495 & 0.329 & 0.884 & 20.3 \\
\hline
\end{tabular}

APPLIED ECOLOGY AND ENVIRONMENTAL RESEARCH 17(3): 6887-6916.

http://www.aloki.hu • ISSN 15891623 (Print) • ISSN 17850037 (Online)

DOI: http://dx.doi.org/10.15666/aeer/1703_68876916

(c) 2019, ALÖKI Kft., Budapest, Hungary 


\begin{tabular}{|c|c|c|c|c|c|c|c|c|c|c|c|c|c|c|c|c|}
\hline \multirow{3}{*}{ Pesticide } & \multicolumn{8}{|c|}{ Cucumber } & \multicolumn{8}{|c|}{ Grapefruit } \\
\hline & \multirow[b]{2}{*}{$\mathbf{U}_{1}$} & \multirow[b]{2}{*}{$\mathbf{U}_{2}$} & \multirow{2}{*}{$\begin{array}{c}\begin{array}{c}\mathrm{U}_{\mathrm{i}}^{\mathrm{a}} \\
(\mathrm{mg} / \mathrm{kg} \\
(\%))\end{array} \\
\mathrm{U}_{3}\end{array}$} & \multirow[b]{2}{*}{$\begin{array}{r}\mathbf{U}_{4} \\
(\mathrm{mg} / \mathrm{kg})\end{array}$} & \multirow[b]{2}{*}{$\mathbf{U}_{6}$} & \multirow[b]{2}{*}{$\mathbf{U}_{\mathbf{c}}$} & \multirow[b]{2}{*}{$\begin{array}{c}\mathbf{U}_{\mathbf{e}}^{\mathbf{b}} \\
(\mathrm{mg} / \mathrm{kg} \\
)\end{array}$} & \multirow[b]{2}{*}{$\begin{array}{c}\mathbf{U}_{\mathbf{e}}(\%) \\
)\end{array}$} & \multirow[b]{2}{*}{$\mathbf{U}_{1}$} & \multirow[b]{2}{*}{$\mathbf{U}_{2}$} & \multirow{2}{*}{$\begin{array}{c}\begin{array}{c}\mathrm{U}_{\mathrm{i}}^{\mathrm{a}} \\
(\mathrm{mg} / \mathrm{kg} \\
(\%))\end{array} \\
\mathrm{U}_{3}\end{array}$} & \multirow[b]{2}{*}{$\begin{array}{r}\mathbf{U}_{4} \\
(\mathrm{mg} / \mathrm{kg})\end{array}$} & \multirow[b]{2}{*}{$\mathbf{U}_{6}$} & \multirow[b]{2}{*}{$\mathbf{U}_{\mathbf{c}}$} & \multirow[b]{2}{*}{$\begin{array}{c}\mathbf{U}_{\mathbf{e}}^{\mathbf{b}} \\
(\mathrm{mg} / \mathrm{kg} \\
)\end{array}$} & \multirow[b]{2}{*}{$\begin{array}{c}\mathrm{U}_{\mathrm{e}}(\% \\
)\end{array}$} \\
\hline & & & & & & & & & & & & & & & & \\
\hline Halfenprox & $0.044(1.9)$ & $0.036(0.8)$ & $0.031(1.5)$ & $0.041(0.5) 0.039(1.8)$ & $0.312(92.4)$ & 0.298 & 0.547 & 18.4 & $0.044(1.9)$ & $0.036(0.8)$ & $0.031(1.5)$ & $0.071(2.1) 0.070$ & $0.452(94.1)$ & 0.385 & 0.771 & 21.6 \\
\hline $\begin{array}{l}\text { Haloxyfop-2- } \\
\text { Etoxyethyl }\end{array}$ & $0.044(2.0)$ & $0.036(1.6)$ & $0.031(1.6)$ & $0.040(0.6) 0.041(2.1)$ & $0.298(89.7)$ & 0.337 & 0.618 & 15.2 & $0.044(2.0)$ & $0.036(1.6)$ & $0.031(1.6)$ & $0.033(1.5) 0.064$ & $0.413(92.1)$ & 0.384 & 0.665 & 23.4 \\
\hline $\begin{array}{l}\text { Haloxyfop-P- } \\
\text { Methyl }\end{array}$ & $0.044(3.4)$ & $0.036(0.5$ & $0.031(1.0)$ & $0.043(0.2) 0.068$ & $0.301(92.1)$ & 0.289 & 0.662 & 16.5 & $0.044(3.4)$ & $0.036(0.9)$ & $0.031(1.0)$ & $0.051(1.9) 0.044$ & $0.402(90.8)$ & 0.394 & 0.882 & 21.8 \\
\hline Hexaconazole & $0.044(3.3)$ & $0.036(1.2)$ & $0.031(1.1)$ & $0.054(0.4) 0.062(2.1)$ & $0.317(89.5)$ & 0.287 & 0.665 & 16.9 & $0.044(3.3)$ & $0.036(1.2)$ & $0.031(1.1)$ & $0.076(2.2) 0.068$ & 0.350 & 0.392 & 0.962 & 22.8 \\
\hline Hexaflumuron & $0.044(3.2)$ & $0.036(1.1)$ & $0.031(1.4)$ & $0.071(0.3) 0.045(1.8)$ & $0.245(90.4)$ & 0.269 & 0.514 & 16.3 & $0.044(3.2)$ & $0.036(1.1)$ & $0.031(1.4)$ & 0.071 & $0.318(87.8)$ & 0.389 & 1.012 & 21.8 \\
\hline Hexythiazox & $0.044(3.1)$ & $0.036(1.5)$ & $0.031(1.3)$ & $0.041(0.5) 0.039(1.8)$ & $0.298(88.7)$ & 0.278 & 0.512 & 16.1 & $0.044(3.1)$ & $0.036(1.5)$ & $0.031(1.3)$ & $0.097(6.3) 0.071$ & $0.333(92.1)$ & 0.374 & 0.669 & 23.6 \\
\hline Imazalil & $0.044(2.1)$ & $0.036(1.6)$ & $0.031(0.9)$ & $0.040(0.6) 0.065$ & $0.291(93.1)$ & 0.259 & 0.628 & 15.9 & $0.044(2.1)$ & 0.036 & $0.031(0.9)$ & $0.096(5.4) 0.062$ & $0.368(91.2)$ & 0.365 & 0.771 & 20.8 \\
\hline Imazamox & $0.044(3.2)$ & $0.036(1.7)$ & $0.031(1.1)$ & $0.043(0.2) 0.048(2.2)$ & $0.285(91.1)$ & 0.339 & 0.624 & 17.5 & $0.044(3.2)$ & $0.036(1.7)$ & $0.031(1.1)$ & $0.071(2.1) 0.045$ & 0.495 & 0.361 & 0.778 & 19.6 \\
\hline Imazapic & $0.044(3.1)$ & $0.036(1.4)$ & $0.031(1.4)$ & $0.054(0.4) 0.048(2.2)$ & $0.331(92.2)$ & 0.341 & 0.701 & 15.4 & $0.044(3.1)$ & $0.036(1.4)$ & $0.031(1.4)$ & $0.033(1.5) 0.061$ & $0.452(94.1)$ & 0.368 & 0.668 & 18.9 \\
\hline Imazapyr & $0.044(2.8)$ & $0.036(1.5)$ & 0.031 & $0.071(0.3) 0.038(1.8)$ & $0.337(92.6)$ & 0.287 & 0.704 & 16.4 & $0.044(2.8)$ & $0.036(1.5)$ & $0.031(1.3)$ & 0.051 & $0.413(92.1)$ & 0.382 & 0.669 & 17.6 \\
\hline Imazethapyr & $0.044(2.7)$ & $0.036(1.2)$ & $0.031(0.9)$ & $0.072(4.3) 0.039(1.8)$ & $0.298(89.7)$ & 0.298 & 0.517 & 15.7 & $0.044(2.7)$ & $0.036(1.2)$ & $0.031(0.9)$ & $0.097(6.3) 0.064$ & $0.402(90.8)$ & 0.381 & 0.770 & 15.9 \\
\hline Imidacloprid & $0.044(2.5)$ & $0.036(1.3)$ & $0.031(0.7)$ & $0.008(4.2) 0.041$ & $0.291(93.1)$ & 0.301 & 0.514 & 15.9 & $0.044(2.5)$ & $0.036(1.3)$ & $0.031(0.7)$ & $0.096(5.4) 0.044$ & $0.350(88.4)$ & 0.504 & 0.776 & 19.8 \\
\hline $\begin{array}{l}\text { Iodosulfuron- } \\
\text { Methyl }\end{array}$ & $0.044(2.3)$ & $0.036(1.0)$ & $0.031(0.8)$ & 0.007 & $0.332(85.6)$ & 0.305 & 0.548 & 17.6 & $0.044(2.3)$ & $0.036(1.0)$ & $0.031(0.8)$ & 0.071 & $0.318(87.8)$ & 0.501 & 0.778 & 18.6 \\
\hline Ioxynil & $0.044(2.1)$ & $0.036(1.3)$ & $0.031(0.9)$ & 0.009 & $0.312(92.4)$ & 0.321 & 0.587 & 18.6 & $0.044(2.1)$ & $0.036(1.3)$ & $0.031(0.9)$ & $0.033(1.5) 0.060$ & $0.333(92.1)$ & 0.359 & 0.776 & 23.6 \\
\hline Iprodione & $0.044(2.0)$ & $0.036(1.0)$ & $0.031(1.0)$ & 0.070 & $0.298(89.7)$ & 0.315 & 0.514 & 17.8 & $0.044(2.0)$ & $0.036(1.0)$ & $0.031(1.0)$ & 0.051 & $0.368(91.2)$ & 0.385 & 0.765 & 20.8 \\
\hline Isoxaflutole & $0.044(3.2)$ & $0.036(0.9)$ & $0.031(1.1)$ & $0.066(0.2) 0.039$ & $0.301(92.1)$ & 0.311 & 0.665 & 17.6 & $0.044(3.2)$ & 0.036 & $0.031(1.1)$ & $0.076(2.2) 0.062$ & 0.495 & 0.504 & 0.765 & 19.6 \\
\hline Kresor & $0.044(3.5)$ & $0.036(1.3)$ & $0.031(1.4)$ & $0.052(0.1) 0.052(3.4)$ & $0.317(89.5)$ & 0.314 & 0.541 & 15.9 & $0.044(3.5)$ & 0.036 & $0.031(1.4)$ & 0.071 & $0.452(94.1)$ & 0.501 & 0.665 & 18.9 \\
\hline $\begin{array}{c}\text { Lambda } \\
\text { Cyhalothrin }\end{array}$ & $0.044(1.9)$ & $0.036(1.0)$ & $0.031(1.3)$ & $0.054(0.6) 0.037$ & $0.245(90.4)$ & 0.317 & 0.526 & 18.1 & $0.044(1.9)$ & $0.036(1.0)$ & $0.031(1.3)$ & $0.097(6.3) 0.061$ & $0.413(92.1)$ & 0.328 & 0.654 & 17.6 \\
\hline Lenacil & $0.044(2.0)$ & $0.036(0.9)$ & $0.031(0.9)$ & $0.051(0.7) 0.045(1.8)$ & $0.298(88.7)$ & 0.298 & 0.668 & 17.6 & $0.044(2.0)$ & $0.036(0.9)$ & $0.031(0.9)$ & $0.096(5.4) 0.070$ & $0.402(90.8)$ & 0.329 & 0.774 & 15.9 \\
\hline Lufenuron & $0.044(3.4)$ & $0.036(0.8)$ & $0.031(0.7)$ & $0.041(0.5) 0.062(2.1)$ & $0.291(93.1)$ & 0.324 & 0.662 & 15.9 & $0.044(3.4)$ & $0.036(0.8)$ & $0.031(0.7)$ & $0.071(2.1) 0.064$ & 0.350 & 0.385 & 0.771 & 19.8 \\
\hline
\end{tabular}

APPLIED ECOLOGY AND ENVIRONMENTAL RESEARCH 17(3): 6887-6916.

http://www.aloki.hu • ISSN 15891623 (Print) • ISSN 17850037 (Online)

DOI: http://dx.doi.org/10.15666/aeer/1703_68876916

(c) 2019, ALÖKI Kft., Budapest, Hungary 


\begin{tabular}{|c|c|c|c|c|c|c|c|c|c|c|c|c|c|c|c|c|}
\hline \multirow{3}{*}{ Pesticide } & \multicolumn{8}{|c|}{ Cucumber } & \multicolumn{8}{|c|}{ Grapefruit } \\
\hline & \multirow[b]{2}{*}{$\mathbf{U}_{1}$} & \multirow[b]{2}{*}{$\mathbf{U}_{2}$} & \multirow{2}{*}{$\begin{array}{c}\begin{array}{c}\mathrm{U}_{\mathrm{i}}^{\mathrm{a}} \\
(\mathrm{mg} / \mathrm{kg} \\
(\%))\end{array} \\
\mathrm{U}_{3}\end{array}$} & \multirow[b]{2}{*}{$\begin{array}{r}\mathbf{U}_{4} \\
(\mathrm{mg} / \mathrm{kg})\end{array}$} & \multirow[b]{2}{*}{$\mathbf{U}_{6}$} & \multirow[b]{2}{*}{$\mathbf{U}_{\mathbf{c}}$} & \multirow[b]{2}{*}{$\begin{array}{c}\mathbf{U}_{\mathbf{e}}^{\mathbf{b}} \\
(\mathrm{mg} / \mathrm{kg} \\
\quad) \\
\end{array}$} & \multirow[b]{2}{*}{$\stackrel{\mathbf{U}_{\mathbf{e}}(\%)}{)}$} & \multirow[b]{2}{*}{$\mathbf{U}_{1}$} & \multirow[b]{2}{*}{$\mathbf{U}_{2}$} & \multirow{2}{*}{$\begin{array}{c}\begin{array}{c}\mathrm{U}_{\mathrm{i}}^{\mathrm{a}} \\
(\mathrm{mg} / \mathrm{kg} \\
(\%))\end{array} \\
\mathrm{U}_{3}\end{array}$} & \multirow[b]{2}{*}{$\begin{array}{r}\mathbf{U}_{4} \\
(\mathrm{mg} / \mathrm{kg})\end{array}$} & \multirow[b]{2}{*}{$\mathbf{U}_{6}$} & \multirow[b]{2}{*}{$\mathbf{U}_{\mathrm{c}}$} & \multirow[b]{2}{*}{$\begin{array}{c}\mathbf{U}_{\mathbf{e}}^{\mathbf{b}} \\
(\mathrm{mg} / \mathrm{kg} \\
\quad) \\
\end{array}$} & \multirow[b]{2}{*}{$\begin{array}{c}\mathbf{U}_{\mathbf{e}}(\%) \\
\quad)\end{array}$} \\
\hline & & & & & & & & & & & & & & & & \\
\hline Malathion & $0.044(3.3)$ & 0.036 & $0.031(0.8)$ & $0.040(0.6) 0.048(2.2)$ & 0.337 & 0.289 & 0.669 & 14.6 & $0.044(3.3)$ & $0.036(1.6)$ & $0.031(0.8)$ & $0.033(1.5) 0.044(1.7)$ & 0.318 & 0.384 & +0.659 & 20.1 \\
\hline MCPA & $0.044(3.2)$ & 0.036 & $0.031(0.9)$ & $0.043(0.2) 0.038(1.8)$ & 0.285 & 0.287 & 0.662 & 15.6 & $0.044(3.2)$ & $0.036(1.2)$ & 0.031 & 0.051 (1.9)0.068 (1.6) & 0.333 & 0.394 & +0.662 & 20.8 \\
\hline Mecoprop & $0.044(3.1)$ & 0.036 & $0.031(1.0)$ & ) $0.054(0.4) 0.039(1.8)$ & 0.329 & 0.269 & 0.547 & 12.3 & $0.044(3.1)$ & $0.036(1.1)$ & 0.031 & $0.076(2.2) 0.060(3.5)$ & $0.368(91.2)$ & 0.392 & 0.652 & 22.8 \\
\hline Mefenpyr-diethyl & $0.044(2.1)$ & 0.036 & $0.031(1.0)$ & ) $0.071(0.3) 0.041(2.1)$ & 0.331 & 0.278 & 0.521 & 14.8 & $0.044(2.1)$ & $0.036(1.5)$ & 0.031 & ) $0.071(4.4) 0.071(3.4)$ & 0.495 & 0.389 & 1.014 & 22.7 \\
\hline $\begin{array}{l}\text { Mesosulfuron } \\
\text { methyl }\end{array}$ & $0.044(3.2)$ & 0.036 & $0.031(1.1)$ & ) $0.041(0.5) 0.068(3.5)$ & 0.285 & 0.259 & 0.562 & 15.4 & $0.044(3.2)$ & $0.036(1.6)$ & 0.031 & ) $0.097(6.3) 0.062(2.6)$ & $0.452(94.1)$ & 0.374 & +0.665 & 21.6 \\
\hline Mesotrione & $0.044(3.1)$ & 0.036 & $0.031(1.4)$ & $0.040(0.6) 0.039(1.8)$ & 0.337 & 0.321 & 0.574 & 14.6 & $0.044(3.1)$ & $0.036(1.7)$ & 0.031 & .) $0.096(5.4) 0.045(1.6)$ & 0.413 & 0.365 & 1.011 & 20.4 \\
\hline Metalaxyl & $0.044(2.8)$ & 0.036 & $0.031(1.3)$ & ) $0.043(0.2) 0.052(3.4)$ & 0.298 & 0.287 & 0.618 & 14.5 & $0.044(2.8)$ & $0.036(1.4)$ & 0.031 & ) $0.071(2.1) 0.061(3.2)$ & 0.402 & 0.361 & 0.774 & 19.8 \\
\hline Metamitron & $0.044(2.1)$ & $0.036(1.5)$ & $0.031(0.9)$ & $0.054(0.4) 0.037$ (1.7) & $0.332(85.6)$ & 0.298 & 0.662 & 17.2 & $0.044(2.1)$ & $0.036(1.5)$ & $0.031(0.9)$ & $0.033(1.5) 0.070$ ( 3.3$)$ & $0.350(88.4)$ & 0.368 & 0.776 & 15.7 \\
\hline Metconazole & $0.044(2.0)$ & $0.036(1.2)$ & $0.031(1.1)$ & ) $0.071(0.3) 0.045(1.8)$ & $0.312(92.4)$ & 0.301 & 0.665 & 17.1 & $0.044(2.0)$ & $0.036(1.2)$ & 0.031 & ) 0.051 (1.9)0.064 (2.8) & 0.318 & 0.501 & 0.669 & 16.4 \\
\hline Methidathion & $0.044(3.2)$ & $0.036(1.3)$ & $0.031(1.4)$ & $0.008(4.2) 0.048(2.2)$ & 0.298 & 0.305 & 0.514 & 15.6 & $0.044(3.2)$ & $0.036(1.3)$ & 0.031 & ) $0.096(5.4) 0.044(1.7)$ & 0.333 & 0.425 & 0.771 & 18.3 \\
\hline Methomyl & $0.044(3.5)$ & $0.036(1.0)$ & $0.031(1.3)$ & ) $0.007(4.1) 0.038(1.8)$ & 0.301 & 0.321 & 0.512 & 18.1 & $0.044(3.5)$ & $0.036(1.0)$ & 0.031 & $0.071(2.1) 0.068(1.6)$ & $0.368(91.2)$ & 0.384 & 0.778 & 19.5 \\
\hline Methoxyfenozide & $0.044(1.9)$ & $0.036(1.3)$ & $0.031(0.9)$ & 0.009 (3.9)0.039 (1.8) & 0.317 & 0.315 & 0.628 & 16.4 & $0.044(1.9)$ & $0.036(1.3)$ & $0.031(0.9)$ & $0.033(1.5) 0.060(3.5)$ & 0.495 & 0.382 & 20.668 & 15.6 \\
\hline Metolachlor & $0.044(2.2)$ & $0.036(1.0)$ & $0.031(0.7)$ & $0.070(3.7) 0.041(2.1)$ & 0.245 & 0.311 & 0.624 & 15.3 & $0.044(2.2)$ & $0.036(1.0)$ & 0.031 & 0.051 (1.9)0.071 (3.4) & 0.452 & 0.381 & 0.669 & 16.2 \\
\hline Metrubuzin & $0.044(2.0)$ & $0.036(0.9)$ & $0.031(0.8)$ & $0.066(0.2) 0.068$ & 0.298 & 0.314 & 0.701 & 15.8 & $0.044(2.0)$ & $0.036(0.9)$ & $0.031(0.8)$ & $0.076(2.2) 0.062(2.6)$ & 0.413 & 0.504 & +0.770 & 19.6 \\
\hline $\begin{array}{l}\text { Metsulfuron- } \\
\text { methyl }\end{array}$ & $0.044(3.4)$ & $0.036(0.8)$ & $0.031(0.9)$ & $0.052(0.1) 0.065(2.6)$ & $0.291(93.1)$ & 0.317 & 0.704 & 15.9 & $0.044(3.4)$ & $0.036(0.8)$ & $0.031(0.9)$ & $0.071(4.4) 0.045(1.6)$ & $0.402(90.8)$ & 0.501 & 0.776 & 20.3 \\
\hline Monocrotophos & $0.044(3.3)$ & $0.036(1.6)$ & $0.031(1.0)$ & $0.054(0.6) 0.048(2.2)$ & 0.285 & 0.298 & 0.517 & 17.6 & $0.044(3.3)$ & $0.036(1.6)$ & 0.031 & $0.097(6.3) 0.061(3.2)$ & 0.350 & 0.359 & 0.778 & 21.6 \\
\hline Monolinuron & $0.044(3.2)$ & $0.036(0.9)$ & $0.031(1.1)$ & ) $0.051(0.7) 0.039(1.8)$ & 0.337 & 0.297 & 0.514 & 18.6 & $0.044(3.2)$ & $0.036(0.9)$ & 0.031 & $0.096(5.4) 0.070$ ( 3.3$)$ & $0.318(87.8)$ & 0.385 & 0.776 & 23.4 \\
\hline Myclobutanil & $0.044(3.1)$ & $0.036(1.2)$ & $0.031(1.4)$ & $0.041(0.5) 0.062(2.1)$ & 0.298 & 0.299 & 0.548 & 17.8 & $0.044(3.1)$ & $0.036(1.2)$ & 0.031 & ) $0.071(2.1) 0.064(2.8)$ & 0.333 & 0.504 & 0.765 & 21.8 \\
\hline Nicosulfuron & $0.044(2.1)$ & $0.036(1.1)$ & $0.031(1.3)$ & ) $0.040(0.6) 0.045(1.8)$ & 0.332 & 0.289 & 0.514 & 17.6 & $0.044(2.1)$ & $0.036(1.1)$ & 0.031 & ) $0.033(1.5) 0.044(1.7)$ & $0.368(91.2)$ & 0.501 & 1.011 & 22.8 \\
\hline Novaluron & $0.044(2.3)$ & $0.036(1.5)$ & $0.031(0.9)$ & $0.043(0.2) 0.048(2.2)$ & 0.312 & 0.287 & 0.665 & 15.9 & $0.044(2.3)$ & $0.036(1.5)$ & $0.031(0.9)$ & 0.051 (1.9)0.068 (1.6) & 0.495 & 0.328 & 30.994 & 21.8 \\
\hline
\end{tabular}

APPLIED ECOLOGY AND ENVIRONMENTAL RESEARCH 17(3): 6887-6916.

http://www.aloki.hu • ISSN 15891623 (Print) • ISSN 17850037 (Online)

DOI: http://dx.doi.org/10.15666/aeer/1703_68876916

(c) 2019, ALÖKI Kft., Budapest, Hungary 


\begin{tabular}{|c|c|c|c|c|c|c|c|c|c|c|c|c|c|c|c|c|}
\hline \multirow{3}{*}{ Pesticide } & \multicolumn{8}{|c|}{ Cucumber } & \multicolumn{8}{|c|}{ Grapefruit } \\
\hline & \multirow[b]{2}{*}{$\mathbf{U}_{1}$} & \multirow[b]{2}{*}{$\mathbf{U}_{2}$} & \multirow{2}{*}{$\begin{array}{c}\begin{array}{c}\mathrm{U}_{\mathrm{i}}^{\mathrm{a}} \\
(\mathrm{mg} / \mathrm{kg}\end{array} \\
(\%))\end{array}$} & \multirow[b]{2}{*}{$\begin{array}{r}\mathbf{U}_{4} \\
\mathrm{~kg})\end{array}$} & \multirow[b]{2}{*}{$\mathbf{U}_{6}$} & \multirow[b]{2}{*}{$\mathbf{U}_{\mathbf{c}}$} & \multirow[b]{2}{*}{$\begin{array}{c}\mathbf{U}_{\mathbf{e}}^{\mathbf{b}} \\
(\mathrm{mg} / \mathrm{kg} \\
)\end{array}$} & \multirow[b]{2}{*}{$\begin{array}{c}\mathbf{U}_{\mathrm{e}}(\%) \\
)\end{array}$} & \multirow[b]{2}{*}{$\mathbf{U}_{1}$} & \multirow[b]{2}{*}{$\mathbf{U}_{2}$} & \multirow{2}{*}{$\begin{array}{c}\begin{array}{c}\mathrm{U}_{\mathrm{i}}^{\mathrm{a}} \\
(\mathrm{mg} / \mathrm{kg} \\
(\%))\end{array} \\
\mathrm{U}_{3}\end{array}$} & \multirow[b]{2}{*}{$\begin{array}{r}\mathbf{U}_{\mathbf{4}} \\
(\mathrm{mg} / \mathrm{kg})\end{array}$} & \multirow[b]{2}{*}{$\mathbf{U}_{6}$} & \multirow[b]{2}{*}{$\mathbf{U}_{\mathbf{c}}$} & \multirow[b]{2}{*}{$\begin{array}{c}\mathbf{U}_{\mathbf{e}}^{\mathbf{b}} \\
(\mathrm{mg} / \mathrm{kg} \\
)\end{array}$} & \multirow[b]{2}{*}{$\begin{array}{c}\mathrm{U}_{\mathrm{e}}(\% \\
)\end{array}$} \\
\hline & & & & & & & & & & & & & & & & \\
\hline Omethoate & $0.044(2.1)$ & $0.036(1.6)$ & $0.031(0.7)$ & $0.054(0.4) 0.038(1.8)$ & $0.298(89.7)$ & 0 & 0.541 & 18.1 & $0.044(2.1)$ & $0.036(1.6)$ & $0.031(0.7)$ & $0.076(2.2) 0.060$ & $0.452(94.1)$ & 0.329 & 0.886 & 23.6 \\
\hline Oxadixyl & $0.044(1.9)$ & $0.036(1.7)$ & $0.031(0.8)$ & $0.071(0.3) 0.039(1.8)$ & $0.301(92.1)$ & 0.278 & 0.526 & 17.6 & $0.044(1.9)$ & $0.036(1.7)$ & $0.031(0.8)$ & 0.071 & $0.413(92.1)$ & 0.385 & 0.887 & 20.8 \\
\hline Oxamyl & $0.044(3.2)$ & $0.036(1.4)$ & $0.031(0.9)$ & $0.041(0.5) 0.041$ & $0.317(89.5)$ & 0.259 & 0.668 & 15.9 & $0.044(3.2)$ & $0.036(1.4)$ & $0.031(0.9)$ & 0.097 & $0.402(90.8)$ & 0.384 & 0.885 & 19.6 \\
\hline Oxyflourfen & $0.044(3.1)$ & $0.036(1.5)$ & $0.031(1.0)$ & $0.040(0.6) 0.068$ & $0.245(90.4)$ & 0.317 & 0.662 & 16.3 & $0.044(3.1)$ & $0.036(1.5)$ & $0.031(1.0)$ & $0.096(5.4) 0.045$ & $0.350(88.4)$ & 0.394 & 0.881 & 18.9 \\
\hline Parathion $\mathrm{I}$ & $0.044(2.8)$ & $0.036(1.2)$ & $0.031(1.4)$ & $0.043(0.2) 0.052(3.4)$ & $0.298(88.7)$ & 0.287 & 0.669 & 15.6 & $0.044(2.8)$ & $0.036(1.2)$ & $0.031(1.4)$ & $0.071(2.1) 0.061$ & $0.318(87.8)$ & 0.392 & 0.669 & 17.6 \\
\hline Penconazole & $0.044(2.1)$ & $0.036(1.3)$ & $0.031(1.3)$ & $0.054(0.4) 0.037(1.7)$ & $0.332(85.6)$ & 0.298 & 0.618 & 16.3 & $0.044(2.1)$ & $0.036(1.3)$ & $0.031(1.3)$ & $0.033(1.5) 0.070$ & $0.333(9$ & 0.389 & 0.771 & 15.9 \\
\hline Pendimethalin & $0.044(2.0)$ & $0.036(1.0)$ & $0.031(0.9)$ & $0.071(0.3) 0.045$ & $0.312(92.4)$ & 0.301 & 0.662 & 15.6 & $0.044(2.0)$ & $0.036(1.0)$ & $0.031(0.9)$ & 0.051 & $0.368(91.2)$ & 0.374 & 0.778 & 18.3 \\
\hline Permethrin & $0.044(3.2)$ & $0.036(1.0)$ & $0.031(1.1)$ & $0.023(0.7) 0.065$ & $0.298(89.7)$ & 0.305 & 0.665 & 18.1 & $0.044(3.2)$ & $0.036(1.0)$ & $0.031(1.1)$ & $0.096(5.4) 0.044$ & $0.495(95.1)$ & 0.365 & 0.668 & 17.5 \\
\hline Phenmedipham & $0.044(3.5)$ & $0.036(0.9)$ & $0.031(1.4)$ & $0.072(4.3) 0.048(2.2)$ & $0.291(93.1)$ & 0.321 & 0.514 & 14.5 & $0.044(3.5)$ & $0.036(0.9)$ & $0.031(1.4)$ & 0.071 & $0.452(94.1)$ & 0.361 & 0.669 & 17.3 \\
\hline Phenthoate & $0.044(1.9)$ & $0.036(1.2)$ & $0.031(1.3)$ & 0.008 & $0.285(91.1)$ & 0.315 & 0.512 & 16.2 & $0.044(1.9)$ & $0.036(1.2)$ & $0.031(1.3)$ & $0.033(1.5) 0.060$ & $0.413(92.1$ & 0.368 & 0.770 & 17.2 \\
\hline Phosalone & $0.044(2.0)$ & $0.036(1.1)$ & $0.031(0.9)$ & $0.007(4.1) 0.039(1.8)$ & $0.329(93.3)$ & 0.311 & 0.628 & 18.2 & $0.044(2.0)$ & $0.036(1.1)$ & $0.031(0.9)$ & $0.051(1.9) 0.071$ & $0.402(90$ & 0.441 & 0.776 & 19.6 \\
\hline Phosmet & $0.044(3.4)$ & $0.036(1.5)$ & $0.031(0.7)$ & 0.009 & $0.337(92.6)$ & 0.314 & 0.624 & 14.3 & 0.044 (3.4) & $0.036(1.5)$ & $0.031(0.7)$ & $0.076(2.2) 0.062$ & $0.350(88.4)$ & 0.445 & 0.778 & 15.6 \\
\hline Pirimicarb & $0.044(3.3)$ & $0.036(1.6)$ & $0.031(0.8)$ & 0.070 & $0.298(89.7)$ & 0.317 & 0.701 & 16.9 & $0.044(3.3)$ & $0.036(1.6)$ & $0.031(0.8)$ & 0.071 & $0.318(87.8)$ & 0.384 & 0.776 & 16.2 \\
\hline Pirimiphos methyl & $0.044(3.2)$ & $0.036(1.7)$ & $0.031(0.9)$ & $0.066(0.2) 0.048(2.2)$ & $0.301(92.1)$ & 0.298 & 0.704 & 14.8 & $0.044(3.2)$ & $0.036(1.7)$ & $0.031(0.9)$ & $0.097(6.3) 0.061$ & $0.333(92.1)$ & 0.382 & 0.765 & 19.6 \\
\hline Prochloraz & $0.044(3.1)$ & $0.036(1.4)$ & $0.031(1.0)$ & $0.052(0.1) 0.038(1.8)$ & $0.317(89.5)$ & 0.315 & 0.517 & 15.9 & $0.044(3.1)$ & $0.036(1.4)$ & $0.031(1.0)$ & 0.096 & $0.368(91.2)$ & 0.381 & 0.765 & 20.3 \\
\hline Procymidone & $0.044(2.1)$ & $0.036(1.5)$ & $0.031(0.8)$ & $0.054(0.6) 0.039(1.8)$ & $0.245(90.4)$ & 0.347 & 0.514 & 17.6 & $0.044(2.1)$ & $0.036(1.5)$ & $0.031(0.8)$ & $0.071(2.1) 0.064$ & $0.495(95$ & 0.504 & 0.665 & 21.6 \\
\hline Profenofos & $0.044(2.3)$ & $0.036(1.2)$ & $0.031(1.5)$ & $0.051(0.7) 0.041$ & $0.298(88.7)$ & 0.325 & 0.548 & 18.6 & $0.044(2.3)$ & $0.036(1.2)$ & $0.031(1.5)$ & $0.033(1.5) 0.044$ & $0.452(94.1)$ & 0.501 & 0.654 & 20.8 \\
\hline $\begin{array}{l}\text { Profoxydim } \\
\text { (Clefoxydim) }\end{array}$ & $0.044(2.1)$ & $0.036(1.3)$ & $0.031(1.6)$ & $0.041(0.5) 0.068(3.5)$ & $0.332(85.6)$ & 0.298 & 0.587 & 17.8 & $0.044(2.1)$ & $0.036(1.3)$ & $0.031(1.6)$ & $0.051(1.9) 0.068$ & $0.413(92.1)$ & 0.359 & 0.774 & 19.6 \\
\hline Prometryn & $0.044(1.9)$ & $0.036(1.0)$ & $0.031(1.0)$ & $0.040(0.6) 0.045$ & $0.312(92.4)$ & 0.289 & 0.514 & 17.6 & $0.044(1.9)$ & $0.036(1.0)$ & $0.031(1.0)$ & $0.076(2.2) 0.060$ & $0.402(90.8)$ & 0.385 & 0.771 & 17.6 \\
\hline Propaquizafop & $0.044(3.2)$ & $0.036(1.3)$ & $0.031(1.1)$ & $0.043(0.2) 0.039(1.8)$ & $0.298(89.7)$ & 0.287 & 0.665 & 15.9 & $0.044(3.2)$ & $0.036(1.3)$ & $0.031(1.1)$ & 0.071 & $0.350(8$ & 0.504 & 0.659 & 18.2 \\
\hline Propargite & $0.044(3.1)$ & $0.036(1.0)$ & $0.031(1.4)$ & $0.054(0.4) 0.052$ & $0.291(93.1)$ & 0.269 & 0.541 & 18.1 & $0.044(3.1)$ & $0.036(1.0)$ & $0.031(1.4)$ & 0.097 & $0.318(87.8)$ & 0.501 & 0.662 & 17.1 \\
\hline
\end{tabular}

APPLIED ECOLOGY AND ENVIRONMENTAL RESEARCH 17(3): 6887-6916.

http://www.aloki.hu • ISSN 15891623 (Print) • ISSN 17850037 (Online)

DOI: http://dx.doi.org/10.15666/aeer/1703_68876916

(c) 2019, ALÖKI Kft., Budapest, Hungary 


\begin{tabular}{|c|c|c|c|c|c|c|c|c|c|c|c|c|c|c|c|c|}
\hline \multirow{3}{*}{ Pesticide } & \multicolumn{8}{|c|}{ Cucumber } & \multicolumn{8}{|c|}{ Grapefruit } \\
\hline & \multirow[b]{2}{*}{$\mathbf{U}_{1}$} & \multirow[b]{2}{*}{$\mathbf{U}_{2}$} & \multirow{2}{*}{$\begin{array}{c}\begin{array}{c}\mathrm{U}_{\mathrm{i}}^{\mathrm{a}} \\
(\mathrm{mg} / \mathrm{kg} \\
(\%))\end{array} \\
U_{3}\end{array}$} & \multirow[b]{2}{*}{$\begin{array}{l}\mathbf{U}_{4} \\
\mathrm{~kg})\end{array}$} & \multirow[b]{2}{*}{$\mathbf{U}_{6}$} & \multirow[b]{2}{*}{$\mathbf{U}_{\mathbf{c}}$} & \multirow[b]{2}{*}{$\begin{array}{c}\mathbf{U}_{\mathbf{e}}^{\mathbf{b}} \\
(\mathrm{mg} / \mathrm{kg} \\
)\end{array}$} & \multirow[b]{2}{*}{$\begin{array}{c}\mathbf{U}_{\mathbf{e}}(\%) \\
)\end{array}$} & \multirow[b]{2}{*}{$\mathbf{U}_{1}$} & \multirow[b]{2}{*}{$\mathbf{U}_{2}$} & \multirow{2}{*}{$\begin{array}{c}\begin{array}{c}\mathrm{U}_{\mathrm{i}}^{\mathrm{a}} \\
(\mathrm{mg} / \mathrm{kg} \\
(\%))\end{array} \\
\mathrm{U}_{3}\end{array}$} & \multirow[b]{2}{*}{$\begin{array}{r}\mathbf{U}_{\mathbf{4}} \\
(\mathrm{mg} / \mathrm{kg})\end{array}$} & \multirow[b]{2}{*}{$\mathbf{U}_{6}$} & \multirow[b]{2}{*}{$\mathbf{U}_{\mathbf{c}}$} & \multirow[b]{2}{*}{$\begin{array}{c}\mathbf{U}_{\mathbf{e}}^{\mathbf{b}} \\
(\mathrm{mg} / \mathrm{kg} \\
)\end{array}$} & \multirow[b]{2}{*}{$\begin{array}{c}\mathrm{U}_{\mathrm{e}}(\% \\
)\end{array}$} \\
\hline & & & & & & & & & & & & & & & & \\
\hline Propazine & $0.044(2.8)$ & $0.036(0.9)$ & 0.031 & $0.071(0.3) 0.037(1.7)$ & $0.285(91.1)$ & 0 & 0.526 & 17.6 & $0.044(2.8)$ & $0.036(0.9)$ & $0.031(1.3)$ & $0.096(5.4) 0.045$ & $0.333(92.1)$ & 0.328 & 0.652 & 18.7 \\
\hline Propoxycarbazone & $0.044(2.1)$ & $0.036(0.8)$ & $0.031(0.9)$ & $0.041(0.5) 0.045(1.8)$ & $0.337(92.6)$ & 0.259 & 0.668 & 15.9 & $0.044(2.1)$ & $0.036(0.8)$ & $0.031(0.9)$ & 0.071 & $0.368(91.2)$ & 0.329 & 1.014 & 18.8 \\
\hline Propyconazole & $0.044(2.0)$ & $0.036(1.6)$ & $0.031(1.1)$ & $0.040(0.6) 0.048(2.2)$ & $0.298(89.7)$ & 0.315 & 0.662 & 18.5 & $0.044(2.0)$ & $0.036(1.6)$ & $0.031(1.1)$ & 0.033 & $0.495(95.1)$ & 0.385 & 0.665 & 16.4 \\
\hline Propyzamide & $0.044(3.2)$ & $0.036(1.2)$ & 0.031 & $0.043(0.2) 0.038(1.8)$ & $0.301(92.1)$ & 0.347 & 0.669 & 14.5 & $0.044(3.2)$ & $0.036(1.2)$ & $0.031(1.4)$ & $0.051(1.9) 0.064$ & $0.452(94.1)$ & 0.384 & 1.011 & 18.3 \\
\hline Prothopos & $0.044(3.5)$ & $0.036(1.1)$ & $0.031(1.3)$ & $0.054(0.4) 0.039(1.8)$ & $0.317(89.5)$ & 0.325 & 0.514 & 16.9 & $0.044(3.5)$ & $0.036(1.1)$ & $0.031(1.3)$ & 0.071 & $0.413(92.1)$ & 0.394 & 0.774 & 19.5 \\
\hline Pymetrozine & $0.044(1.9)$ & $0.036(1.5)$ & $0.031(0.9)$ & $0.071(0.3) 0.041$ & $0.245(90.4)$ & 0.317 & 0.618 & 15.6 & $0.044(1.9)$ & $0.036(1.5)$ & $0.031(0.9)$ & $0.097(6.3) 0.068$ & $0.402(9$ & 0.392 & 0.776 & 15.6 \\
\hline Pyraclostrobin & $0.044(2.0)$ & $0.036(1.6)$ & $0.031(0.7)$ & $0.008(4.2) 0.068$ & $0.298(88.7)$ & 0.315 & 0.662 & 18.2 & $0.044(2.0)$ & $0.036(1.6)$ & $0.031(0.7)$ & $0.096(5.4) 0.060$ & $0.350(88.4)$ & 0.389 & 0.669 & 16.2 \\
\hline Pyraflufen-Ethyl & $0.044(3.4)$ & $0.036(1.7)$ & $0.031(0.8)$ & 0.007 & $0.332(85.6)$ & 0.347 & 0.665 & 17.6 & $0.044(3.4)$ & $0.036(1.7)$ & $0.031(0.8)$ & 0.071 & $0.318(87.8)$ & 0.374 & 0.771 & 19.6 \\
\hline Pyrazophos & $0.044(3.3)$ & $0.036(1.4)$ & $0.031(0.9)$ & 0.009 & $0.312(92.4)$ & 0.325 & 0.514 & 13.6 & $0.044(3.3)$ & $0.036(1.4)$ & $0.031(0.9)$ & $0.033(1.5) 0.062$ & $0.333(92.1)$ & 0.365 & 0.778 & 20.3 \\
\hline Pyridaben & $0.044(3.2)$ & $0.036(1.5)$ & $0.031(1.0)$ & 0.070 & $0.298(89.7)$ & 0.287 & 0.512 & 15.2 & $0.044(3.2)$ & $0.036(1.5)$ & $0.031(1.0)$ & $0.051(1.9) 0.045$ & $0.368(91.2)$ & 0.361 & 0.668 & 21.6 \\
\hline Pyridaphention & $0.044(3.1)$ & $0.036(1.2)$ & $0.031(1.1)$ & $0.066(0.2) 0.065$ & $0.291(93.1)$ & 0.298 & 0.628 & 14.6 & $0.044(3.1)$ & $0.036(1.2)$ & $0.031(1.1)$ & $0.076(2.2) 0.061$ & $0.495(95.1)$ & 0.368 & 0.669 & 23.4 \\
\hline Pyridate & $0.044(2.1)$ & $0.036(1.3)$ & 0.031 & $0.052(0.1) 0.039(1.8)$ & $0.285(91.1)$ & 0.301 & 0.624 & 12.5 & $0.044(2.1)$ & $0.036(1.3)$ & $0.031(1.4)$ & 0.071 & $0.452(94.1)$ & 0.384 & 0.770 & 21.8 \\
\hline Pyriproxyfen & $0.044(2.3)$ & $0.036(1.0)$ & $0.031(1.3)$ & $0.054(0.6) 0.065$ & $0.337(92.6)$ & 0.305 & 0.701 & 13.6 & $0.044(2.3)$ & $0.036(1.0)$ & $0.031(1.3)$ & $0.097(6.3) 0.064$ & $0.413(92.1)$ & 0.382 & 0.776 & 22.8 \\
\hline $\begin{array}{l}\text { Quizalofop-P- } \\
\text { Ethyl }\end{array}$ & $0.044(2.1)$ & $0.036(1.3)$ & $0.031(0.9)$ & $0.051(0.7) 0.048$ & $0.298(89.7)$ & 0.321 & 0.704 & 14.8 & $0.044(2.1)$ & $0.036(1.3)$ & $0.031(0.9)$ & $0.096(5.4) 0.044$ & $0.402(90.8)$ & 0.381 & 0.778 & 21.8 \\
\hline Rimsulfuron & $0.044(1.9)$ & $0.036(1.0)$ & $0.031(0.7)$ & $0.041(0.5) 0.048(2.2)$ & $0.301(92.1)$ & 0.315 & 0.517 & 15.9 & $0.044(1.9)$ & $0.036(1.0)$ & $0.031(0.7)$ & $0.071(2.1) 0.068$ & $0.350(88.4)$ & 0.504 & 0.776 & 23.6 \\
\hline Sethoxydim & $0.044(3.2)$ & $0.036(0.9)$ & $0.031(0.8)$ & $0.040(0.6) 0.038$ & $0.317(89.5)$ & 0.311 & 0.514 & 17.6 & $0.044(3.2)$ & $0.036(0.9)$ & $0.031(0.8)$ & $0.033(1.5) 0.060$ & $0.318(87.8)$ & 0.501 & 0.765 & 20.8 \\
\hline Simozine & $0.044(3.1)$ & $0.036(0.8)$ & $0.031(0.9)$ & $0.043(0.2) 0.039(1.8)$ & $0.245(90.4)$ & 0.314 & 0.548 & 18.6 & $0.044(3.1)$ & $0.036(0.8)$ & $0.031(0.9)$ & $0.051(1.9) 0.071$ & $0.333(92.1)$ & 0.359 & 0.884 & 19.6 \\
\hline Spirodiclofen & $0.044(2.8)$ & $0.036(1.6)$ & $0.031(1.0)$ & $0.054(0.4) 0.041$ & $0.298(88.7)$ & 0.317 & 0.514 & 15.6 & $0.044(2.8)$ & $0.036(1.6)$ & $0.031(1.0)$ & $0.076(2.2) 0.062$ & $0.368(91.2)$ & 0.385 & 0.765 & 18.9 \\
\hline Spiroxamine & $0.044(2.3)$ & $0.036(1.1)$ & $0.031(0.8)$ & $0.071(0.3) 0.068$ & $0.332(85.6)$ & 0.298 & 0.665 & 15.2 & $0.044(2.3)$ & $0.036(1.1)$ & $0.031(0.8)$ & 0.071 & $0.495(95.1)$ & 0.504 & 0.665 & 17.6 \\
\hline Sulfos & $0.044(2.1)$ & $0.036(1.5)$ & $0.031(1.5)$ & $0.041(0.5) 0.070$ & $0.312(92.4)$ & 0.315 & 0.541 & 17.8 & $0.044(2.1)$ & $0.036(1.5)$ & $0.031(1.5)$ & $0.097(6.3) 0.061$ & $0.452(9$ & 501 & 0.654 & 15.9 \\
\hline Tau-Fluvalinate & $0.044(2.0)$ & $0.036(1.6)$ & 0.031 & $0.040(0.6) 0.039(1.8)$ & $0.298(89.7)$ & 0.347 & 0.526 & 17.9 & $0.044(2.0)$ & $0.036(1.6)$ & $0.031(1.6)$ & 0.096 & ) $0.413(92$. & 0.328 & 0.774 & 20.6 \\
\hline
\end{tabular}

APPLIED ECOLOGY AND ENVIRONMENTAL RESEARCH 17(3): 6887-6916.

http://www.aloki.hu • ISSN 15891623 (Print) • ISSN 17850037 (Online)

DOI: http://dx.doi.org/10.15666/aeer/1703_68876916

(c) 2019, ALÖKI Kft., Budapest, Hungary 


\begin{tabular}{|c|c|c|c|c|c|c|c|c|c|c|c|c|c|c|c|c|}
\hline \multirow{3}{*}{ Pesticide } & \multicolumn{8}{|c|}{ Cucumber } & \multicolumn{8}{|c|}{ Grapefruit } \\
\hline & \multirow[b]{2}{*}{$\mathbf{U}_{1}$} & \multirow[b]{2}{*}{$\mathbf{U}_{2}$} & \multirow{2}{*}{$\begin{array}{c}\begin{array}{c}\mathrm{U}_{\mathrm{i}}^{\mathrm{a}} \\
(\mathrm{mg} / \mathrm{kg}\end{array} \\
(\%))\end{array}$} & \multirow[b]{2}{*}{$\begin{array}{l}\mathbf{U}_{4} \\
\mathrm{~kg})\end{array}$} & \multirow[b]{2}{*}{$\mathbf{U}_{6}$} & \multirow[b]{2}{*}{$\mathbf{U}_{\mathbf{c}}$} & \multirow[b]{2}{*}{$\begin{array}{c}\mathbf{U}_{\mathbf{e}}^{\mathbf{b}} \\
(\mathrm{mg} / \mathrm{kg} \\
)\end{array}$} & \multirow[b]{2}{*}{$\begin{array}{c}\mathbf{U}_{\mathbf{e}}(\% \\
)\end{array}$} & \multirow[b]{2}{*}{$\mathbf{U}_{1}$} & \multirow[b]{2}{*}{$\mathbf{U}_{2}$} & \multirow{2}{*}{$\begin{array}{c}\begin{array}{c}\mathrm{U}_{\mathrm{i}}^{\mathrm{a}} \\
(\mathrm{mg} / \mathrm{kg} \\
(\%))\end{array} \\
\mathrm{U}_{3}\end{array}$} & \multirow[b]{2}{*}{$\begin{array}{r}\mathbf{U}_{4} \\
(\mathrm{mg} / \mathrm{kg})\end{array}$} & \multirow[b]{2}{*}{$\mathbf{U}_{6}$} & \multirow[b]{2}{*}{$\mathbf{U}_{\mathbf{c}}$} & \multirow[b]{2}{*}{$\begin{array}{c}\mathbf{U}_{\mathbf{e}}^{\mathbf{b}} \\
(\mathrm{mg} / \mathrm{kg} \\
) \\
\end{array}$} & \multirow[b]{2}{*}{$\begin{array}{c}\mathbf{U}_{\mathbf{e}}(\% \\
\quad)\end{array}$} \\
\hline & & & & & & & & & & & & & & & & \\
\hline Tebucor & $0.044(3.2)$ & $0.036(1.7)$ & $0.031(1.0)$ & $0.043(0.2) 0.052(3.4)$ & $0.291(93.1)$ & 0.325 & 0.668 & 12.3 & $0.044(3.2)$ & $0.036(1.7)$ & $0.031(1.0)$ & $0.071(2.1) 0.064(2.8)$ & $0.402(90.8)$ & 0.329 & 0.771 & 22.3 \\
\hline Tebufenozide & $0.044(3.5)$ & $0.036(1.4)$ & $0.031(1.1)$ & $0.054(0.4) 0.037(1.7)$ & $0.337(92.6)$ & 0.314 & 0.662 & 15.6 & $0.044(3.5)$ & $0.036(1.4)$ & $0.031(1.1)$ & $0.033(1.5) 0.044(1.7)$ & $0.350(88$ & 0.385 & 0.659 & 22.9 \\
\hline Teflubenzuron & $0.044(1.9)$ & $0.036(1.5)$ & $0.031(1.4)$ & $0.071(0.3) 0.045(1.8)$ & $0.298(89.7)$ & 0.289 & 0.669 & 14.3 & $0.044(1.9)$ & $0.036(1.5)$ & $0.031(1.4)$ & $0.051(1.9) 0.068(1.6)$ & $0.318(87.8)$ & 0.384 & 0.662 & 21.8 \\
\hline Tepraloxydim & $0.044(2.0)$ & $0.036(1.2)$ & $0.031(1.3)$ & $0.041(0.5) 0.048(2.2)$ & $0.301(92.1)$ & 0.287 & 0.662 & 15.6 & $0.044(2.0)$ & $0.036(1.2)$ & $0.031(1.3)$ & $0.076(2.2) 0.060(3.5)$ & $0.333(92.1)$ & 0.394 & 0.652 & 15.6 \\
\hline Terbuthylazine & $0.044(3.4)$ & $0.036(1.3)$ & $0.031(0.9)$ & $0.040(0.6) 0.038(1.8)$ & $0.317(89.5)$ & 0.269 & 0.665 & 18.5 & $0.044(3.4)$ & $0.036(1.3)$ & $0.031(0.9)$ & $0.071(4.4) 0.071(3.4)$ & $0.368(91.2)$ & 0.392 & 1.014 & 16.2 \\
\hline Terbutryn & $0.044(3.3)$ & $0.036(1.0)$ & $0.031(0.7)$ & $0.043(0.2) 0.039(1.8)$ & $0.245(90.4)$ & 0.278 & 0.514 & 14.3 & $0.044(3.3)$ & $0.036(1.0)$ & $0.031(0.7)$ & $0.097(6.3) 0.062(2.6)$ & ) $0.495(95$ & 0.389 & 0.665 & 19.6 \\
\hline Tetraconazole & $0.044(3.2)$ & $0.036(1.3)$ & $0.031(0.8)$ & $0.054(0.4) 0.041(2.1)$ & $0.298(88.7)$ & 0.259 & 0.512 & 15.2 & $0.044(3.2)$ & $0.036(1.3)$ & $0.031(0.8)$ & $0.096(5.4) 0.045(1.6)$ & $0.452(94.1)$ & 0.374 & 1.011 & 20.3 \\
\hline Thiabendazole & $0.044(3.1)$ & $0.036(1.0)$ & $0.031(0.9)$ & $0.071(0.3) 0.068(3.5)$ & $0.289(88.9)$ & 0.311 & 0.628 & 15.6 & $0.044(3.1)$ & $0.036(1.0)$ & $0.031(0.9)$ & $0.071(2.1) 0.061(3.2)$ & $0.413(92.1)$ & 0.365 & 0.774 & 21.6 \\
\hline Thiacloprid & $0.044(2.1)$ & 0.036 & 0.031( & $0.007(4.1) 0.052(3.4)$ & $0.288(96.5)$ & 0 & 0 & 18.1 & 0.044 & 0.0 & $0.031(1.0)$ & $0.033(1.5) 0.070(3.3)$ & 4 & 0.361 & 0.776 & 19.5 \\
\hline Thiametl & $0.044(2.3)$ & $0.036(0.8)$ & 0.031 & 0.009 & $0.291(93.1)$ & 0.298 & 0.701 & 14.5 & $0.044(2.3)$ & 0.036 & 0.031 & $0.051(1.9) 0.064(2.8)$ & $0.350(8$ & 0.368 & 0.669 & 18.2 \\
\hline $\begin{array}{l}\text { Thifensulfuron- } \\
\text { methyl }\end{array}$ & $0.044(2.1)$ & $0.036(1.6)$ & $0.031(1.4)$ & 0.070 & $0.332(85.6)$ & 0.301 & 0.704 & 16.7 & $0.044(2.1)$ & $0.036(1.6)$ & 0.031 & $0.076(2.2) 0.044(1.7)$ & $0.318(87.8)$ & 0.381 & 0.771 & 18.1 \\
\hline $\begin{array}{c}\text { Thiohanate } \\
\text { methyl }\end{array}$ & $0.044(1.9)$ & $0.036(1.1)$ & 0.031 & $0.066(C$ & $2(92.4)$ & 05 & 0.517 & 16.9 & $0.044(1.9)$ & $0.036(1.1)$ & 0.031 & $0.071(4.4) 0.068(1.6)$ & ) $0.333(92$ & 0.504 & 0.778 & 17.3 \\
\hline Thiometon & $0.044(3.2)$ & $0.036(1.5)$ & $0.031(0.9)$ & $0.052(0.1) 0.039(1.8)$ & $0.298(89.7)$ & 0.321 & 0.514 & 17.6 & $0.044(3.2)$ & $0.036(1.5)$ & 0.031 & $0.097(6.3) 0.060(3.5)$ & $0.368(91.2)$ & 0.501 & 0.668 & 17.1 \\
\hline Tolyfluanid & $0.044(3.1)$ & $0.036(1.6)$ & $0.031(0.7)$ & $0.054(0.6) 0.052(3.4)$ & $0.332(94.6)$ & 0.315 & 0.548 & 17.2 & $0.044(3.1)$ & $0.036(1.6)$ & $0.031(0.7)$ & $0.096(5.4) 0.071(3.4)$ & $0.495(95.1)$ & 0.359 & 0.669 & 15.7 \\
\hline Tralkoxydim & $0.044(2.8)$ & $0.036(1.7)$ & $0.031(0.8)$ & $0.051(0.7) 0.037(1.7)$ & $0.301(92.1)$ & 0.311 & 0.623 & 15.9 & $0.044(2.8)$ & $0.036(1.7)$ & $0.031(0.8)$ & $0.071(2.1) 0.062(2.6)$ & $0.452(94.1)$ & 0.385 & 0.765 & 16.4 \\
\hline Triadimefon & $0.044(2.4)$ & $0.036(1.4)$ & $0.031(0.9)$ & $0.041(0.5) 0.045(1.8)$ & $0.317(89.5)$ & 0.314 & 0.654 & 14.8 & $0.044(2.4)$ & $0.036(1.4)$ & $0.031(0.9)$ & $0.033(1.5) 0.045(1.6)$ & $0.413(92$. & 0.504 & 0.665 & 18.3 \\
\hline Triadimenol & $0.044(3.1)$ & $0.036(1.5)$ & $0.031(0.8)$ & $0.040(0.6) 0.074$ & $0.245(90.4)$ & 0.317 & 0.681 & 15.9 & $0.044(3.1)$ & $0.036(1.5)$ & $0.031(0.8)$ & $0.051(1.9) 0.061(3.2)$ & $0.402(90.8)$ & 0.501 & 0.654 & 19.5 \\
\hline Triallate & $0.044(2.3)$ & $0.036(1.2)$ & 0.031 & $0.043(0.2) 0.048(2.2)$ & $0.298(88.7)$ & 0.298 & 0.618 & 17.6 & $0.044(2.3)$ & $0.036(1.2)$ & 0.031 & $0.034(1.2) 0.070$ & $0.350(88.4)$ & 0.328 & 0.774 & 15.6 \\
\hline Triasulfuron & $0.044(2.1)$ & $0.036(1.3)$ & 0.031 & $0.054(0.4) 0.038(1.8)$ & $0.335(95.6)$ & 0.301 & 0.662 & 18.6 & 040 & 036 & 0.031 & $0.076(2.2) 0.064$ & $0.318(8$ & 0.329 & 0.771 & 16.2 \\
\hline $\begin{array}{l}\text { Tribenuron- } \\
\text { Methyl }\end{array}$ & 0 & 0 & 0 & $0.071(0.3) 0.039(1.8)$ & 5.6) & 0.308 & 65 & 17.8 & ) & 026 & 21 & $0.071(4.4) 0.044$ & ) 0.333 & 0.385 & 0.659 & 19.6 \\
\hline Trichlorfon & $0.044(3.2)$ & $0.036(1.0)$ & 0.031 & $0.008(4.2) 0.041(2.1)$ & $0.312(92.4)$ & 0.296 & 0.514 & 17.6 & $0.044(3.2)$ & $0.036(1.0)$ & 0.031 & $0.097(6.3) 0.068(1.6)$ & $0.368(91.2)$ & 0.384 & 0.662 & 20 \\
\hline
\end{tabular}

APPLIED ECOLOGY AND ENVIRONMENTAL RESEARCH 17(3): 6887-6916.

http://www.aloki.hu • ISSN 15891623 (Print) • ISSN 17850037 (Online)

DOI: http://dx.doi.org/10.15666/aeer/1703_68876916

(c) 2019, ALÖKI Kft., Budapest, Hungary 


\begin{tabular}{|c|c|c|c|c|c|c|c|c|c|c|c|c|c|c|c|c|c|}
\hline \multirow{3}{*}{ Pesticide } & \multicolumn{8}{|c|}{ Cucumber } & \multicolumn{9}{|c|}{ Grapefruit } \\
\hline & \multirow[b]{2}{*}{$\mathbf{U}_{1}$} & \multirow[b]{2}{*}{$\mathbf{U}_{2}$} & \multirow{2}{*}{$\begin{array}{c}\begin{array}{c}\mathbf{U}_{\mathrm{i}}^{\mathrm{a}} \\
(\mathbf{m g} / \mathbf{k g} \\
(\%))\end{array} \\
\mathrm{U}_{3} \\
(1\end{array}$} & \multirow[b]{2}{*}{$\begin{array}{r}\mathbf{U}_{4} \\
(\mathrm{mg} / \mathrm{kg})\end{array}$} & \multirow[b]{2}{*}{$\mathbf{U}_{6}$} & \multirow[b]{2}{*}{$\mathbf{U}_{\mathbf{c}}$} & \multirow[b]{2}{*}{$\begin{array}{c}\mathbf{U}_{\mathbf{e}}^{\mathbf{b}} \\
(\mathrm{mg} / \mathrm{kg} \\
\quad) \\
\end{array}$} & \multirow[b]{2}{*}{$\begin{array}{c}\mathbf{U}_{\mathbf{e}}(\%) \\
)\end{array}$} & \multirow[b]{2}{*}{$\mathbf{U}_{1}$} & \multirow[b]{2}{*}{$\mathbf{U}_{2}$} & \multirow{2}{*}{$\begin{array}{c}\begin{array}{c}\mathrm{U}_{\mathrm{i}}^{\mathrm{a}} \\
(\mathrm{mg} / \mathrm{kg} \\
(\%))\end{array} \\
\mathrm{U}_{3} \\
\end{array}$} & \multirow[b]{2}{*}{$\begin{array}{r}\mathbf{U}_{4} \\
(\mathrm{mg} / \mathrm{kg})\end{array}$} & \multirow[b]{2}{*}{$\mathbf{U}_{5}$} & \multirow[b]{2}{*}{$\mathbf{U}_{6}$} & \multirow[b]{2}{*}{$\mathbf{U}_{\mathrm{c}}$} & \multirow[b]{2}{*}{$\begin{array}{c}\mathbf{U}_{\mathbf{e}}^{\mathbf{b}} \\
(\mathrm{mg} / \mathrm{kg} \\
)\end{array}$} & \multirow[b]{2}{*}{$\begin{array}{c}\mathbf{U}_{\mathbf{e}}(\% \\
)\end{array}$} \\
\hline & & & & & & & & & & & & & & & & & \\
\hline Trifloxystrobin & $0.044(3.5)$ & $0.036(0.9)$ & 0.031 & $0.007(4.1) 0.068(3.5)$ & $0.298(89.7)$ & 0.289 & 0.512 & 15.9 & $0.044(3.5)$ & $0.036(0.9)$ & 0.031 & $0.096(5.4)$ & $0.060(3.5) c$ & $0.495(95.1)$ & 0.394 & 0.652 & 21.6 \\
\hline Triflumizole & $0.044(1.9)$ & $0.036(1.2)$ & 0.031 & 0.009 & $0.301(92.1)$ & 0.287 & 0.628 & 18.1 & $0.044(1.9)$ & $0.036(1.2)$ & 0.031 & $0.071(2.1)$ & $0.071(3.4) c$ & $0.452(94.1)$ & 0.392 & 1.014 & 23.4 \\
\hline Triflumuron & $0.044(2.3)$ & $0.036(1.1)$ & $0.031(0.9)$ & 0.070 & $0.317(89.5)$ & 0.269 & 0.624 & 17.6 & $0.044(2.3)$ & $0.036(1.1)$ & 0.031 & $0.033(1.5)$ & $0.062(2.6) \mathrm{C}$ & $0.413(92.1)$ & 0.389 & 0.665 & 21.8 \\
\hline Triticonazole & $0.044(2.1)$ & $0.036(1.5)$ & $0.031(1.5)$ & $0.066(0.2) 0.037(1.7)$ & $0.245(90.4)$ & 0.278 & 0.701 & 15.9 & $0.044(2.1)$ & $0.036(1.5)$ & $0.031(1.5)$ & $0.051(1.9)$ & $0.045(1.6) \mathrm{C}$ & $0.402(90.8)$ & 0.374 & 1.011 & 22.8 \\
\hline 2.4 D Acid & $0.044(1.9)$ & $0.036(1.6)$ & $0.031(0.8)$ & $0.052(0.1) 0.045$ & $0.298(88.7)$ & 0.259 & 0.704 & 18.7 & $0.044(1.9)$ & $0.036(1.6)$ & $0.031(0.8)$ & 0.071 & $0.061(3.2) \mathrm{C}$ & $0.452(94.1)$ & 0.365 & 0.774 & 21.8 \\
\hline
\end{tabular}

$\mathrm{Ui}^{\mathrm{a}}$ are the contributions of each uncertainty source to $\mathrm{U}_{\mathrm{c}}$ of the method.

$\mathrm{Ue}^{\mathrm{b}}$ is the expanded uncertainty for a $4 \mathrm{mg} / \mathrm{kg}$ estimated for a level of confidence of $95 \%$ 


$$
\text { - } 6914 \text { - }
$$

\section{Conclusions}

In this paper, an analytical method for the separation of 233 pesticide in cucumber and grapefruits using QuEChERS method and GC-MS, LC-MS/MS is presented. The validation data showed satisfactory performance of analytical method, in terms of specificity, sensitivity, linearity, recovery, precision and $U_{e}$ according to the European SANTE/11945/2015 Guideline. One or multiple pesticide residues were detected in $11.6 \%$ of cucumber and $13.8 \%$ of grapefruit samples, but the levels were below the EU MRLs. The residues found in cucumbers and grapefruits were belonging to the group of fungicides, insecticides, herbicide, acaricide and plant growth regulator. The most frequently detected residues in cucumber samples were Clofentezine (4.4\%), propyzamide (3.96), propamocarb (3.03), Azinphosethyl (1.8) ,metalxyl, procymidone, chlorpyrifos and Carbendazim. However, the fungicide propamocarb $(8.8 \%)$ was the most common residue in cucumbers, followed by propyzamide $(6.3 \%)$ and Clofentezine $(2 \%)$. The fungicide fludioxonil was the most prominent pesticide found in grapefruit samples, with a frequency of $9.1 \%$. Imazalil was the second most commonly found pesticide in grapefruits with $4.7 \%$ of the samples, followed by Thiabendazol, quinophos and carbaryl. The proposed method was also found to be suitable for different kinds of fruits.

\section{REFERENCES}

[1] Alvarez, M. V., Cabred, S., Ramirez, C. L., Fanovich, M. A. (2019): Valorization of an agroindustrial soybean residue by supercritical fluid extraction of phytochemical compounds. - The Journal of Supercritical Fluids 143: 90-96.

[2] AOAC International (Association of Official Analytical Chemists). (2007): Pesticide Residues in Foods by Acetonitrile Extraction and Partitioning with Magnesium Sulphate. - AOAC Official method 2007.01.

[3] Bakırcı, G. T., Acay, D. B. Y., Bakırcı, F., Ötleş, S. (2014): Pesticide residues in fruits and vegetables from the Aegean region, Turkey. - Food Chem. 160: 379-392.

[4] Bhat, J. A., Rashid, R., Dar, W. A., Bhat, R. A. (2018): Efficacy of Different Fungicides for the Management of Downy Mildew of Cucumber Grown Under Low Plastic Tunnel. - Int. J. Pure App. Biosc. 6(2): 884-890.

[5] Campone, L., Piccinelli, A. L., Celano, R., Pagano, I., Russo, M., Rastrelli, L. (2018): Rapid and automated on-line solid phase extraction HPLC-MS/MS with peak focusing for the etermination of ochratoxin A in wine samples. - Food chemistry 244: 128-135.

[6] Cengiz, M. F., Başlar, M., Basancelebi, O., Kılıçl1, M. (2018): Reduction of pesticide residues from tomatoes by low intensity electrical current and ultrasound applications. Food chemistry 267: 60-66.

[7] Chan, K. K., Hamid, M. S. B., Webster, R. D. (2018): Quantification of capsaicinoids in chillies by solid-phase extraction coupled with voltammetry. - Food Chemistry 265: 152158.

[8] da Silva Sousa, J., de Castro, R. C., de Albuquerque Andrade, G., Lima, C. G., Lima, L. K., Milhome, M. A. L., do Nascimento, R. F. (2013): Evaluation of an analytical methodology using QuEChERS and GC-SQ/MS for the investigation of the level of pesticide residues in Brazilian melons. - Food Chem. 141: 2675e81.

[9] Dong, H., Xian, Y., Xiao, K., Wu, Y., Zhu, L., He, J. (2019): Development and comparison of single-step solid phase extraction and QuEChERS clean-up for the analysis of 7 mycotoxins in fruits and vegetables during storage by UHPLC-MS/MS. Food Chemistry 274: 471-479. 


$$
-6915-
$$

[10] Du, L. J., Chu, C., Warner, E., Wang, Q. Y., Hu, Y. H., Chai, K. J., Cao, J., Peng, L. Q., Chen, Y. B., Yang, J., Zhang, Q. D. (2018): Rapid microwave-assisted dispersive microsolid phase extraction of mycotoxins in food using zirconia nanoparticles. - Journal of Chromatography A. 1561: 1-12.

[11] Epstein, L., Zhang, M. (2014): The impact of integrated pest management programs on pesticide use in California, USA. - In: Peshin, R., Pimentel, D. (eds.) Integrated pest management: experiences with implementation, global overview. Netherlands 4: 173-200.

[12] Ersoy, N., Tekinarslan, O., Ozgür, E. A., Göktas, U. (2018): Determination of Pesticide Residues in Apricot (Prunus armeniaca L.) Grown at Good Agricultural Practices (GAPs) by LC-MS/MS and GC-MS. - Erwerbs-Obstbau 60: 349-358.

[13] European Commission. (2008): Review Report for the Active Substance Boscalid. SANCO 3919/2007-rev.5. Finalised in the Standing Committee on the Food Chain and Animal Health at its Meeting on 22 January 2008 in View of the Inclusion of Boscalid in Annex I of Directive 91/414/EEC.

[14] European Commission. (2015): Guidance Document on Analytical Quality Control and Method Validation Procedures for Pesticides Residues Analysis in Food and Feed. Document No. SANTE/11945/2015 European Commission Directorate-General for Health and Food Safety. Available from: http://ec.europa.eu/food/plant/doc/ plant_pesticides_mrl_guidelines_wrkdoc_11945_en.pdf (cited 2017 Jul 25).

[15] FAO. (2012): FAO specifications and Evaluations for Agricultural Pesticides. Propamocarb, pp. 42. Available from: http://www.fao.org/agriculture/crops/corethemes/theme/pests/jmps/en/ (cited 2018 Jul 23).

[16] FAO. (2015): FAO statistical databases and data sets. - Available from: http://faostat.fao. org (cited 2018 Jan. 23).

[17] Golge, O., Kabak, B. (2015): Determination of 115 pesticide residues in oranges by highperformance liquid chromatography-triple-quadrupole mass spectrometry in combination with QuEChERS. - Journal of Food Composition and Analysis 41: 86-97.

[18] Golge, O., Hepsag, F., Kabak, B. (2018): Health risk assessment of selected pesticide residues in green pepper and cucumber. - Food and Chemical Toxicology 121: 51-64.

[19] Jiang, Z., Cao, X., Li, H., Zhang, C., El-Aty, A. M. A., Jeong, J. H., Shao, Y., Shao, H., Jin, M., Jin, F., Wang, J. (2018): Rapid analysis of tristyrylphenol ethoxylates in cucumber-field system using supercritical fluid chromatography-tandem mass spectrometry. - Food Chemistry 266: 119-125.

[20] Khan, Z., Kamble, N., Bhongale, A., Girme, M., Chauhan, V. B., Banerjee, K. (2018): Analysis of pesticide residues in tuber crops using pressurised liquid extraction and gas chromatography-tandem mass spectrometry. - Food Chemistry 241: 250-257.

[21] Kilic, S., Tongur, T., Kilic, M., Erkaymaz, T. (2018): Determination of Some EndocrineDisrupting Metals and Organochlorinated Pesticide Residues in Baby Food and Infant Formula in Turkish Markets. - Food Analytical Methods 11: 3352-3361.

[22] Kumar, M., Chand, R., Shah, K. (2018): Mycotoxins and Pesticides: Toxicity and Applications in Food and Feed. - Microbial Biotechnology 2: 207-252.

[23] Li, G. J., Wu, H. J., Wang, Y., Hung, W. L., Rouseff, R. L. (2019): Determination of citrus juice coumarins, furanocoumarins and methoxylated flavones using solid phase extraction and HPLC with photodiode array and fluorescence detection. - Food Chemistry 271: 29-38.

[24] Liu, W., Quan, J., Hu, Z. (2018): Detection of Organophosphorus Pesticides in Wheat by Ionic Liquid-Based Dispersive Liquid-Liquid Microextraction Combined with HPLC. Journal of analytical methods in chemistry. https://doi.org/10.1155/2018/8916393.

[25] Polat, A., Polat, S., Simsek, A., Kurt, T. T., Ozyurt, G. (2018): Pesticide residues in muscles of some marine fish species and seaweeds of Iskenderun Bay (Northeastern Mediterranean), Turkey. - Environmental Science and Pollution Research 25: 3756-3764. 
[26] Polyiem, W., Naksen, W., Prapamontol, T. (2018): Gas chromatographic-flame photometric detection of organophosphate pesticide residues and its application in real vegetable and fruit samples from Chiang. - Chiang Mai J. Sci. 45(4): 1933-1943.

[27] Razzaghi, N., Ziarati, P., Rastegar, H., Shoeibi, S., Amirahmadi, M., Conti, G. O., Ferrante, M., Fakhri, Y., Khaneghah, A. M. (2018): The concentration and probabilistic health risk assessment of pesticide residues in commercially available olive oils in Iran. Food Chem. Toxicol. 120: 32-40.

[28] Salemi, A., Khaleghifar, N., Mirikaram, N. (2019): Optimization and comparison of membrane-protected micro-solid-phase extraction coupled with dispersive liquid-liquid microextraction for organochlorine pesticides using three different sorbents. Microchemical Journal 144: 215-220.

[29] Stachniuk, A. L. C. (2018): MS/MS determination of pesticide residues in fruits and vegetables. - Bioactive Molecules in Food 14: 1-26.

[30] Su, M., Jia, L., Wu, X., Sun, H. (2018): Residue investigation of some phenylureas and tebuthiuron herbicides in vegetables by ultra performance liquid chromatography coupled with integrated selective accelerated solvent extraction-clean up in situ. - Journal of the Science of Food and Agriculture 98: 4845-4853.

[31] Tankiewicz, M. (2019): Determination of Selected Priority Pesticides in High Water Fruits and Vegetables by Modified QuEChERS and GC-ECD with GC-MS/MS Confirmation. - Molecules 24: 417.

[32] Tian, Z., Wang, Z., Han, X., Wang, N., Wang, R. (2018): Study on the interaction between cannabinol and DNA using acridine orange as a fluorescence probe. - Journal of Molecular Recognition 31: 2682.

[33] TUIK (Turkish Statistical Institute). (2015): The summary of agricultural statistics. Available from: http://tuik.gov.tr (cited 2018 June 17).

[34] Van Leeuwen, T., Vontas, J., Tsagkarakou, A., Dermauw, W., Tirry, L. (2010): Acaricide resistance mechanisms in the two-spotted spider mite Tetranychus urticae and other important Acari: a review. - Insect biochemistry and molecular biology 40(8): 563-572.

[35] Walorczyk, S. (2014): Validation and use of a QuEChERS - based gas chromatographice tandem mass spectrometric method for multiresidue pesticide analysis in blackcurrants including studies of matrix effects and estimation of measurement uncertainty. - Talanta 120: 106-13.

[36] Wang, K., Xie, X., Zhang, Y., Huang, Y., Zhou, S., Zhang, W., Lin, Y., Fan, H. (2018): Combination of microwave-assisted extraction and ultrasonic-assisted dispersive liquidliquid microextraction for separation and enrichment of pyrethroids. - Food chemistry 240: 1233-1242.

[37] Yıldırım, E., Macun, H. C., Yalçınkaya, İ., Kocasarı, F. Ș., Ekici, H. (2018): Survey of aflatoxin residue in feed and milk samples in Kirıkkale province, Turkey. - Ankara Üniversitesi Veteriner Fakültesi Dergisi 65: 99-204.

[38] Zhu, B., Xu, X., Luo, J., Jin, S., Chen, W., Liu, Z., Tian, C. (2019): Simultaneous determination of 131 pesticides in tea by on-line GPC-GC-MS/MS using graphitized multi-walled carbon nanotubes as dispersive solid phase. - Food chemistry 276: 202-208. 